\title{
Balanced Input from the tRNA Prenyltransferase MiaA Controls the Stress Resistance and Virulence Potential of Extraintestinal Pathogenic Escherichia coli
}

Matthew G. Blango ${ }^{\star \dagger}$, Brittany A. Fleming ${ }^{* \dagger}$, William M. Kincannon ${ }^{\ddagger}$, Alex Tran*, Adam J. Lewis*, Colin W. Russell*, Qin Zhou*, Lisa M. Baird§, John R. Brannonđ, Connor J. Beebout", Vahe Bandarian $¥$, Maria Hadjifraniskou", Michael T. Howard§, and Matthew A. Mulvey*\#

*Division of Microbiology and Immunology, Pathology Department, University of Utah School of Medicine, Salt Lake City, Utah.

‡Department of Chemistry, University of Utah, Salt Lake City, Utah.

§Department of Human Genetics, University of Utah, Salt Lake City, Utah.

"Department of Pathology, Microbiology, and Immunology, Vanderbilt University Medical Center, Nashville, Tennessee.

${ }^{\circ}$ Current address: Junior Research Group RNA Biology of Fungal Infections, Leibniz Institute for Natural Product Research and Infection Biology (Leibniz-HKI), Jena, Germany.

†These authors contributed equally to the work. Listed alphabetically.

\#Corresponding author:

Phone: 801-581-5967

E-mail: mulvey@path.utah.edu 


\section{ABSTRACT}

2 An ability to adapt to rapidly changing and often hostile environments is key to the success

3 of many bacterial pathogens. In Escherichia coli, the highly conserved enzymes MiaA

4 and MiaB mediate the sequential prenylation and methylthiolation of adenosine-37 within

5 tRNAs that decode UNN codons. Here, we show that MiaA, but not MiaB, is critical to the

6 fitness and virulence of extraintestinal pathogenic E. coli (ExPEC), a major cause of

7 urinary tract and bloodstream infections. Deletion of miaA has pleiotropic effects,

8 rendering ExPEC especially sensitive to stressors like nitrogen and oxygen radicals and

9 osmotic shock. We find that stress can stimulate striking changes in miaA expression,

10 which in turn can increase translational frameshifting and markedly alter the bacterial

11 proteome. Cumulatively, these data indicate that ExPEC, and likely other organisms, can

12 vary MiaA levels as a means to fine-tune translation and the spectrum of expressed

13 proteins in response to changing environmental challenges. 


\section{INTRODUCTION}

The translation of mRNA into protein by ribosomes and aminoacyl-transfer RNA

21 (tRNA) complexes is an energy-intensive process that is subject to multiple levels of

22 complicated regulation. For example, tRNAs can be covalently modified by more than 100

23 different moieties that can influence the charging of tRNAs with amino acids, tRNA

24 stability, codon usage, and reading frame maintenance [1-4]. In Escherichia coli and other

25 bacteria, the hypomodification of tRNAs can result in decreased growth rates, altered

26 metabolic requirements, and reduced stress resistance [5-8]. Loss of tRNA modifications

27 can also impact the fitness and virulence potential of many important bacterial pathogens,

28 including Streptomyces pyogenes, Pseudomonas spp., Shigella flexneri, Agrobacterium

29 tumefaciens, Mycobacterium tuberculosis, Aeromonas hydrophila, Streptococcus spp.,

30 and Salmonella enterica serotype Typhimurium [6, 9-21]. Together, these findings

31 suggest that tRNA modification serves as a regulatory nexus that can control a wide array

32 of bacterial activities.

One of the most commonly modified tRNA residues in bacteria is adenosine-37 (A-

34 37), which lies adjacent to the anticodon loop [8, 22]. In its final form in E. coli, A-37 of

35 UNN-recognizing tRNA molecules is oftentimes prenylated and methylthiolated [23]. The

36 miaA gene of $E$. coli encodes a tRNA prenyltransferase that catalyzes the addition of a

37 prenyl group onto the $N^{6}$-nitrogen of $A-37$ to create $i^{6} A-37$ tRNA [24, 25] (Fig. 1A). The

38 modified $\mathrm{i}^{6} \mathrm{~A}-37$ residue is subsequently methylthiolated by the radical-S-

39 adenosylmethionine enzyme MiaB to create $\mathrm{ms}^{2}{ }^{6} \mathrm{~A}-37$ [26]. The bulky and hydrophobic

$40 \mathrm{~ms}^{2}{ }^{6} \mathrm{~A}-37$ modification enhances tRNA interactions with UNN target codons, promoting

41 reading frame maintenance and translational fidelity $[5,8,27]$. Mutations in the miaA locus 
42 result in an unmodified $\mathrm{A}-37$ residue, as prenylation is required for methylthiolation by

43 MiaB. In K-12 laboratory-adpated E. coli strains, mutations in miaA impair attenuation of

44 the tryptophan and phenylalanine operons [28, 29] and diminish translation of the

45 stationary phase sigma factor RpoS and the small RNA chaperone Hfq [7, 30, 31].

46 Additionally, mutants lacking miaA are unable to effectively resolve aberrant DNA-protein

47 crosslinks [32] and have somewhat elevated spontaneous mutation frequencies [33-35].

48 The $\mathrm{ms}^{2} \mathrm{i}^{6} \mathrm{~A}-37$ modification is highly conserved in both prokaryotes and eukaryotes,

49 though the specific enzymes that mediate this modification have diverged within

50 evolutionarily distant organisms [8]. However, in prokaryotes, MiaA and MiaB

51 homologues are relatively well conserved, and the enzymes appear to function similarly

52 in all tested bacterial species [36, 37].

Given that the $\mathrm{ms}^{2}{ }^{6} \mathrm{~A}-37$ modification is a well-defined regulator of many tRNA

54 functions in lab-adapted K-12 E. coli strains, we sought to understand how this

55 modification is co-opted in a pathogenic E. coli background. E. coli pathotypes display

56 extensive genetic diversity and are usually more resilient under stress than their lab-

57 adapted counterparts [38]. Extraintestinal Pathogenic E. coli (ExPEC) typically reside in

58 the lower intestinal tract of mammals, where they are rarely associated with pathology

59 [39]. However, when they spread outside the gut to other host sites ExPEC can cause a

60 number of serious diseases, including urinary tract and bloodstream infections [38, 40].

61 Bacterial pathogens like ExPEC must be able to rapidly respond to a diverse array of

62 stressors encountered within changing host environments. These include nutrient

63 deprivation, redox stress in the form of oxygen and nitrogen radicals, extremes in $\mathrm{pH}$, 
64 envelope damage, changing osmotic pressures, and a wide assortment of host immune

65 effector cells and antimicrobial compounds [41-45].

Shifts in the prevalence of specific tRNA modifications, such as A-37 prenylation

67 mediated by MiaA, are proposed to help optimize bacterial responses to stress by

68 affecting translational fidelity and selective protein expression [14, 33, 46]. In other words,

69 changing levels of tRNA modifications may control the codon-biased translation of select

70 transcripts, providing a post-transcriptional programmable mechanism that distressed

71 cells can use to facilitate beneficial changes in their proteomes. Here we provide evidence

72 in support of this hypothesis, showing that ExPEC can modulate MiaA levels in response

73 to stress, and that varying levels of this enzyme can increase translational frameshifting

74 and markedly alter the spectrum of expressed proteins. Furthermore, our data reveal that

75 MiaA, but not MiaB, is critical to the fitness and virulence of ExPEC in both in vitro assays

76 and in mouse models of infection and intestinal colonization.

RESULTS

\section{MiaA promotes ExPEC fitness and virulence in vivo}

To assess the importance of MiaA and MiaB for ExPEC within varied host

81 environments, we employed well-established mouse models of gut colonization, urinary

82 tract infection (UTI), and bloodstream infection [47]. For these and subsequent

83 experiments, miaA and miaB were independently deleted from the ExPEC reference

84 strain UTI89 to generate the isogenic knockout mutants UTI89 $\Delta m i a A$ and UTI89 $\Delta m i a B$

$85[48,49]$ 
Gut colonization. The mammalian gastrointestinal $(\mathrm{Gl})$ tract serves as a major

87 reservoir for ExPEC that can seed extraintestinal infections [50-54]. Roles for MiaA and

88 MiaB in ExPEC colonization of the Gl tract were probed using competitive assays in which

$89 \sim 10^{9}$ colony forming units (CFU) of a $1: 1$ mixture of UTI89 and either UTI89 $\mathrm{miaA}$ or

90 UTI89 $\triangle$ miaB were introduced into adult specific-pathogen-free (SPF) BALB/c mice via

91 intragastric gavage [55-57]. In this model system, the levels of ExPEC recovered from the

92 feces reflect ExPEC titers within the large intestines [55]. For these assays, UTI89 and

93 the miaA and miaB knockout mutants were engineered to express either kanamycin

$94\left(\mathrm{Kan}^{\mathrm{R}}\right)$ or chloramphenicol $\left(\mathrm{Cam}^{\mathrm{R}}\right)$ resistance cassettes so that the strains could be

95 readily identified by plating fecal homogenates on selective media. Feces were collected

96 at the indicated time points and the numbers of viable bacteria were enumerated to

97 determine competitive indices $(\mathrm{Cl})$. UTI89 $\Delta$ miaA was significantly outcompeted by wild-

98 type UTI89 as early as day 3 post-inoculation (Fig. 1B). By day 10, there was about a

9925,000 -fold reduction in the relative levels of UTI89 $\Delta$ miaA recovered from the feces,

100 correlating with a median $\mathrm{Cl}$ of -4.39 . At this time point, UTI89 $\Delta$ miaA titers in the majority

101 of mice were below the limit of detection. In contrast, there were no notable differences

102 in titers between UTI89 $\triangle$ miaB and UTI89 in the feces at any time point (Fig. 1C). These

103 results indicate that the loss of MiaA, but not MiaB, greatly impairs the fitness of UTI89

104 within the gut

105 UTI. During the course of a UTI, ExPEC is able to bind and invade the host 106 epithelial cells that comprise the bladder mucosa [58]. Once internalized into bladder

107 cells, ExPEC can traffic into late endosome-like compartments where it may form 108 quiescent reservoir populations that promote long-term bacterial persistence. 
109 Alternatively, ExPEC can enter the host cytosol and rapidly multiply, forming large

110 intracellular bacterial communities that eventually rupture the epithelial cell. In cell culture-

111 based assays using a bladder epithelial cell line, we found that UTI89 $\Delta$ miaA and

112 UTI89 $\Delta$ miaB are able to bind, invade, and survive intracellularly in overnight assays much

113 like wild-type UTI89 (Supplemental Fig. S1).

114 To investigate MiaA and MiaB requirements during UTI, $10^{7} \mathrm{CFU}$ of wild-type

115 UTI89, UTI89 $\triangle$ miaA, and UTI89 $\Delta$ miaB were independently inoculated via transurethral

116 catheterization into adult female CBA/J mice and bacterial titers in the bladders were

117 determined after 3 days. In this analysis, UTI89 $\Delta$ miaB showed no statistically significant

118 defect relative to the parent strain; whereas the $\Delta m i a A$ strain was clearly attenuated (Fig.

119 1D). Deficiencies in bladder colonization by UTI89 $\Delta$ miaA were apparent by $6 \mathrm{~h}$ post-

120 inoculation (Supplemental Fig. S2A), and were still significant after 9 days

121 (Supplemental Fig. S2B). The differences observed between wild-type UTI89 and

$122 \mathrm{UTI} 89 \mathrm{miaA}$ at 3 days post-inoculation of CBA/J mice were also manifest in $\mathrm{C} 3 \mathrm{H} / \mathrm{HeJ}$

123 mice (Supplemental Fig. S2C). Due to defects in Toll-like receptor 4 (TLR4) signaling

124 and other innate defenses, $\mathrm{C} 3 \mathrm{H} / \mathrm{HeJ}$ mice have attenuated inflammatory responses and

125 increased susceptibility to UTI [59-62]. Our results indicate that the decreased capacity

126 of UTI89 $\triangle$ miaA to colonize the bladder is not attributable to an inability of the miaA

127 knockout to handle TLR4-dependent innate host defenses. Collectively, these results

128 indicate that MiaA is required for maximal fitness in mouse UTI models, while MiaB is less

129 critical.

130 Bloodstream infection. ExPEC is a leading cause of bloodstream infections, which

131 too often trigger discordant systemic inflammatory responses that can result in a life- 
132 threatening condition known as sepsis [63]. To examine the contributions of MiaA and

133 MiaB to ExPEC virulence and fitness in a model of sepsis, adult C57Bl/6 mice were

134 inoculated via intraperitoneal (i.p.) injections with $\sim 10^{7}$ CFU of wild-type UTI89,

135 UTI89 $\Delta$ miaA, or UTI89 $\Delta$ miaB. Following i.p. injection, the bacteria enter the bloodstream

136 and disseminate $[56,64,65]$. In our experiments, only $15 \%(2 / 13)$ of the mice infected

137 with wild-type UTI89, and 0\% (0/13) of the mice injected with UTI89 $\Delta$ miaB, were viable

138 after 48 hours (Fig. 1E). In sharp contrast, 84\% (11/13) of the mice infected with

139 UTI89 $\Delta$ miaA survived. At six hours post-injection, significantly lower numbers of bacteria

140 were recovered from the spleens and kidneys of UTI89 $\Delta$ miaA-infected mice, relative to

141 mice infected with wild-type UTI89 or UTI89 1 miaB (Supplemental Fig. S3A and B).

142 While not significant, titers in the liver also trended lower in UTI89 $\Delta$ miaA-infected mice

143 (Supplemental Fig. S3C). Combined, these data demonstrate that MiaA is important for

144 the virulence of ExPEC and its survival during systemic infections, while MiaB appears

145 dispensable.

MiaA enhances EXPEC growth and stress resistance

149 moderately impaired in nutrient-rich broth, but less so in nutrient-limited media [27, 35,

150 66]. Using in vitro growth assays, we found that UTI89 $\Delta$ miaA grew normally in modified

151 M9 minimal media, but failed to reach densities as high as the wild-type strain in more

152 complex, nutrient-rich lysogeny broth (LB) (Fig. 2A and B). In contrast, the miaB knockout

153 exhibited no overt growth defects in either type of media. These data suggest that

154 UTI89 $\triangle$ miaA has reduced metabolic flexibility relative to wild-type UTI89 and the miaB 
155 mutant. This may contribute to the decreased fitness of UTI89 $\Delta$ miaA in our mouse

156 models, where the bacteria likely encounter marked shifts in nutrient availability.

157 However, within different host environments ExPEC will face a wide variety of additional

158 challenges that might be countered by MiaA-dependent processes. We investigated this

159 possibility by examining the effects of MiaA and MiaB on ExPEC resistance to nitrosative,

160 oxidative, and osmotic stress.

Oxidative and nitrosative stress. During the course of an infection, both host and

162 bacterial cells can produce reactive oxygen and nitrogen radicals that can damage lipids, 163 proteins, and nucleic acids $[67,68]$. The contributions of MiaA and MiaB to nitrosative

164 and oxidative stress resistance were assessed using acidified sodium nitrite (ASN) and

165 methyl viologen (MV), respectively. When added to low pH morpholineethanesulfonic acid

166 (MES)-buffered LB (MES-LB; pH 5.0), sodium nitrite dismutates to form nitrous acid which

167 in turn generates $\mathrm{NO}$ and other harmful reactive nitrogen species [69]. In un-

168 supplemented MES-LB, UTI89 2 miaA reached a lower maximal density than wild-type

169 UTI89 (Fig. 2C), similar to results obtained using standard LB (Fig. 2B). The addition 1 $170 \mathrm{mM}$ ASN delayed entry of UTI89 $\mathrm{miaA}$ into exponential growth phase by close to 4 hours

171 (Fig. 2D), while $2 \mathrm{mM} \mathrm{ASN}$ delayed growth by more than 15 hours relative to wild-type

172 UTI89 (Fig. 2E). The addition of $1 \mathrm{mM} \mathrm{MV}$, which produces superoxide radicals [70], had

173 even stronger inhibitory effects on growth of UTI89 $\Delta$ miaA (Fig. 2F). In contrast,

174 UTI89 $\triangle$ miaB grew much like the wild-type strain in the presence of ASN or MV (Fig. 2D-

175 F). Complementation with pMiaA $\mathrm{A}_{\text {nat, }}$ a low copy plasmid that encodes MiaA under control

176 of its native promoter, restored growth of UTI89 $\Delta$ miaA to near wild-type levels in both 2

$177 \mathrm{mM}$ ASN (Fig. 2G) and in $1 \mathrm{mM} \mathrm{MV} \mathrm{(Fig.} \mathrm{2H).}$ 
Osmotic stress. During a UTI, osmotic pressure within the bladder can shift from 50 to $>1,400 \mathrm{mOsm} / \mathrm{kg}$ due to varying concentrations of solutes like sodium and urea [71,

180 72]. By comparison, the normal osmolarity of blood ranges from about 275 to 295 $181 \mathrm{mOsm} / \mathrm{kg}$. To test the sensitivities of wild-type UTI89 and the knockout strains to 182 hypoosmotic stress, we diluted the bacteria from early stationary phase cultures into $183 \mathrm{ddH}_{2} \mathrm{O}$, and then quantified the number of viable bacteria every 30 minutes over the 184 course of 2 hours. Titers of UTI89 2 miaA carrying the empty vector pACYC184 were 185 greatly reduced following exposure to hypoosmotic stress, whereas the levels of UTI89/pACYC184 and UTI89 18 miaB/pACYC184 remained mostly unchanged (Fig. 3A).

187 Survival of UTI89 $\Delta$ miaA was restored by complementation with pMiaA $A_{\text {nat. }}$ To ensure that 188 reduced survival of UTI89 $\Delta$ miaA was attributable to hypoosmotic stress and not 189 starvation, cells were resuspended in $\mathrm{ddH}_{2} \mathrm{O}$ containing $0.1 \%$ glucose, which is 190 comparable to the glucose levels within our M9 medium. Viable bacteria measured after 191120 minutes indicated that the death of UTI89 1 miaA was not due to nutrient deprivation

192 (Fig. 3B). We also observed that UTI89 2 miaA grew poorly in hyperosmotic conditions, 193 created by addition of $5 \% \mathrm{NaCl}$ to standard LB (Fig. 3C). As in other assays, UTI89 $\Delta m i a B$

194 behaved more like the wild-type strain. Growth of UTI89 $\Delta$ miaA was restored to wild-type 195 levels by complementation with pMiaAnat (Fig. 3D). These results indicate that the $m i a A$ 196 knockout has decreased resistance to both hypo- and hyperosmotic stresses.

\section{Hyperosmotic stress attenuates MiaA translation}

We next examined how MiaA levels in UTI89 change in response to environmental 
201 number plasmid (pMiaA-Flagnat) that encodes C-terminal FLAG-tagged MiaA under

202 control of the native miaA promoter. Mid-logarithmic phase cultures of UTI89/pMiaA-

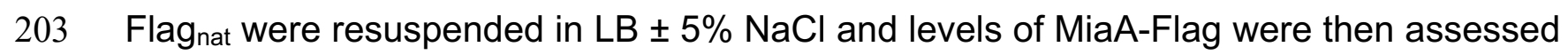

204 by western blot at 30-minute intervals over the course of 1.5 hours (Fig. 4A). Interestingly,

205 MiaA levels in UTI89 exposed to high salt broth were decreased at all time points in

206 comparison with bacteria grown in standard LB. We observed a similar phenomenon if

207 overnight cultures of UTI89/pMiaA-Flagnat in standard LB were back-diluted into high salt

208 broth and then grown to mid-logarithmic phase $\left(\mathrm{OD}_{600} \approx 0.5\right.$, Fig. 4B). Of note, in these

209 assays we observed no loss of the pMiaA-Flagnat construct. In addition, miaA transcripts

210 were often elevated following exposure of UTI89 to high salt (Fig. 4C), suggesting that

211 the downregulation of MiaA protein levels in response to this osmotic stress occurs via a

212 post-transcriptional mechanism. The transcription of miaB was notably reduced under the

213 same conditions (Fig. 4D).

214 To determine if lower amounts of the MiaA protein detected in high salt broth

215 culture affected $\mathrm{i}^{6} \mathrm{~A}$ or $\mathrm{ms}^{2} \mathrm{i}^{6} \mathrm{~A}$ levels, we employed liquid chromatography-coupled mass

216 spectrometry (LC-MS). Normalized amounts of the $i^{6} \mathrm{~A}$ modification in wild-type UT89

217 grown to mid-logarithmic phase in LB were similar to those measured in UTI89 grown in

218 high salt broth (Fig. 4E). However, hyperosmotic stress caused a marked reduction in

219 relative $\mathrm{ms}^{2}{ }^{6}{ }^{\mathrm{A}}$ levels (Fig. 4F), possibly due to reduced transcription of miaB (Fig. 4D).

$220 \mathrm{i}^{6} \mathrm{~A}$ and $\mathrm{ms}^{2} \mathrm{i}^{6} \mathrm{~A}$ were undetectable in UTI89 $\Delta$ miaA, regardless of high salt exposure,

221 confirming that MiaA is required for both modifications (Fig. 4E-F). In contrast, deletion

222 of $m i a B$ prevented formation of $\mathrm{ms}^{2} \mathrm{i}^{6} \mathrm{~A}$, but led to greatly elevated levels of $\mathrm{i}^{6} \mathrm{~A}$

223 (Supplemental Fig. S4). Cumulatively, these data indicate that in response to 
224 hyperosmotic stress UTI89 can post-transcriptionally downregulate MiaA, coordinate with

225 reduction of both $\mathrm{miaB}$ messages and $\mathrm{ms}^{2} \mathrm{i}^{6} \mathrm{~A}$ levels.

\section{Overexpression of MiaA is detrimental under stressful conditions.}

231 utilized a plasmid (pRR48) with miaA under control of an IPTG-inducible Ptac promoter

232 in wild-type UTI89. By LC-MS, relative intensities of $\mathrm{i}^{6} \mathrm{~A}$ were significantly higher in

233 UTI89/pMiaAptac induced with $1 \mathrm{mM}$ IPTG and grown to mid-logarithmic phase in LB

234 compared to UTI89 carrying the empty vector pRR48, whereas the relative intensities of $235 \mathrm{~ms}^{2}{ }^{6}{ }^{6} \mathrm{~A}$ were only modestly elevated (Fig. 5A).

Next, overnight cultures of UTI89/pMiaA ${ }_{\text {Ptac }}$ were back-diluted into LB, LB + $1 \mathrm{mM}$

$237 \mathrm{MV}, \mathrm{LB}+5 \% \mathrm{NaCl}$, MES-LB, or MES-LB $+1 \mathrm{mM} \mathrm{ASN}$, and grown in the presence of

238 increasing IPTG concentrations (Fig. 5B-F). Lower levels of MiaA protein induction

239 caused no overt defects and the bacteria grew much like UTI89/pRR48. However, higher

240 levels of IPTG-induced MiaA expression hindered growth of UTI89/pMiaAptac in the

241 presence of $1 \mathrm{mM} \mathrm{MV}, 5 \% \mathrm{NaCl}, \mathrm{MES}-\mathrm{LB}$, and $1 \mathrm{mM}$ ASN. In contrast, over expression

242 of MiaB did not affect bacteria growth in these in vitro assays (Supplemental Fig. S5).

243 These findings indicate that too much MiaA can be detrimental to bacterial fitness, similar

244 to the complete absence of the enzyme. 
Previous research in K-12 E. coli and Salmonella showed that deletion of miaA can compromise translational fidelity, resulting in increased ribosomal frameshifting [73, 74]. To determine the effects of MiaA on frameshifting in UTI89, we utilized dual-luciferase

250 reporter plasmids that consist of a translational fusion of firefly luciferase downstream of

251 renilla luciferase. Linker sequences, derived from either Antizyme 1 (Az1) or HIV gag-pol,

252 were placed between the two luciferase genes (Fig. 6A). The Az1-derived linker

253 sequence contains a stop codon positioned in-frame so that a +1 frameshift must occur

254 for read-through expression of firefly luciferase [75]. In contrast, a -1 frameshift is required

255 for expression of firefly luciferase downstream of the HIV-derived linker [76]. Importantly,

256 upstream of the in-frame stop codons in both linkers are UNN codons that can be

257 recognized by MiaA-modified tRNAs. The firefly and renilla luciferases act on distinct

258 substrates, which are used to sequentially assess levels of expression of each enzyme

$259[75,76]$. Control plasmids in which the two luciferases are in-frame were used to 260 normalize the data by accounting for ribosome drop-off.

261 To examine the consequences of MiaA expression on frameshifting, the dual262 luciferase reporter constructs were used in combination with wild-type UTI89, 263 UTI89 4 miaA, UTI89/pMiaA ${ }_{\text {Ptac }}$, and UTI89 carrying the empty control vector pRR48. After 264 overnight growth in LB, UTI89 and UTI89 4 miaA were back-diluted into LB while 265 UTI89/pMiaA ${ }_{\text {Ptac }}$ and UTI89/pRR48 were back-diluted into LB + $1 \mathrm{mM}$ IPTG to induce 266 MiaA expession. After reaching mid-log growth, the enzymatic activities of the two 267 luciferases were quantified. Both the lack of MiaA and MiaA overexpression caused 268 notable increases in frameshifting in both the +1 and -1 directions (Fig. 6B-C). These 
269 results confirm that loss of MiaA can increase frameshifting and show that elevated MiaA

270 levels can likewise impact the fidelity of translation.

\section{Changing levels of MiaA alters the spectrum of expressed proteins}

To determine how deletion and overexpression of MiaA affect translation we used

274 multidimensional protein identification technology (MudPIT; LC-MS/MS) with wild-type

275 UTI89 and UTI89 $\Delta$ miaA cultures grown to mid-log phase in LB, and UTI89/pMiaA Ptac and

276 UTI89/pRR48 similarly grown in LB + 1mM IPTG. Of 1,524 proteins detected in UTI89

277 and UTI89 $\triangle$ miaA, 105 were picked up only in the wild-type strain and 23 were unique to

278 the miaA knockout mutant (Fig. 7A). 1,471 proteins were identified in UTI89/pRR48 and

279 UTI89/pMiaA $A_{\text {tac }}$, with 42 being exclusive to UTI89/pRR48 and 20 seen only in the MiaA

280 overexpression strain (Fig. 7B). 115 proteins were significantly downregulated in

281 UTI89 $\triangle$ miaA relative to wild-type UTI89, while 34 proteins were upregulated in the

282 knockout mutant (Fig. 7C). Notably fewer proteins were significantly altered when MiaA

283 was overexpressed. Relative to the control strain UTI89/pRR48, 20 proteins were

284 downregulated in UTI89/pMiaAptac, whereas nine (including MiaA) were upregulated (Fig.

285 7D).

286 The specific proteins detected, including those that were differentially expressed

287 due to miaA deletion or overexpression, are detailed in Supplemental Dataset S1. The

288 differentially expressed proteins were assigned to one or more of 14 functional categories

289 (see Categories worksheet and embedded graph in Supplemental Dataset S1). A

290 majority of the altered proteins were linked with metabolic pathways, secondary

291 metabolites, and functions associated with the bacterial envelope. These included several 
292 proteins involved in sugar and fatty acid metabolism and the biosynthesis and regulation

293 of electron transport chains (e.g. UbiC, WrbA, ChrR, Qor, NuoM, NudJ, and CyoC). The

294 dysregulation of these factors likely contributed to the various phenotypic defects

295 observed in our in vitro and in vivo assays and suggested MiaA involvement in other

296 important processes.

297 In particular, many of the differentially expressed proteins were shown in previous

298 studies to directly or indirectly affect motility or biofilm development. The former group

299 comprised the chemotaxis protein CheA and the flagella-associated proteins FliF, FlhA,

300 and $\mathrm{FlgH}$. Not unexpectedly, both deletion of the miaA gene and MiaA overexpression

301 markedly decreased UTI89 motility on swim plates (Supplemental Fig. S6). MiaB did not

302 affect motility in these assays. Factors linked with biofilm development include YoaB, the

303 type 1 pilus-associated regulator FimB and periplasmic chaperone FimC, the acid stress-

304 response chaperone $\mathrm{HdeB}$, the cellulose synthase catalytic subunit BcsA, and the

305 cytochrome bo subunit CyoC. Using yeast extract-casamino acids (YESCA) medium,

306 which promotes the development of elaborate rugose-colony biofilms [77, 78], we found

307 that UTI89 $\Delta$ miaA, but not UTI89 $\Delta$ miaB, formed atypical biofilms with notably less rugosity

308 than the parent strain (Fig. 8). Interestingly, the biofilms formed by UTI89 $\Delta m i a A$ were

309 architecturally similar to those formed by a UTI89 mutant lacking the CyoC-interacting

310 partners CyoAB [78].

311 Our MudPIT results also indicated that MiaA can regulate numerous proteins that

312 have been associated with redox and bacterial responses to nitrosative, oxidative, and

313 more generally, genotoxic stresses (Supplemental Dataset S1). Aberrant expression of

314 these factors, including proteins like GadB, CadA, Dps, glutathione S-transferase Gst, 
315 and the glutatredoxins GrxB and GrxC, may account for increased sensitivity to oxygen

316 and nitrogen radicals (see Fig. 2D-H and Fig. 5D and F). Some of these factors, and

317 others like HdeA and HdeB, can also guard against acid stress. Accordingly, follow-up

318 experiments confirmed that UTI89 $\Delta$ miaA, but not UTI89 $\Delta$ miaB, is notably less resistant

319 to acid stress than the wild-type strain (Supplemental Fig. S7). On average, relative to

320 wild-type UTI89, UTI89

321 acidic conditions in LB.

The sensitivity of both UTI89 $\Delta$ miaA and UTI89/pMiaAptac to osmotic stress may

323 arise due to the significant downregulation of proteins like SLP, BetB, YggT, ProP, and

324 Ynal (Supplemental Dataset S1). Other differentially expressed proteins that probably

325 contribute to the varied phenotypes associated with miaA deletion or MiaA

326 overexpression in our assays include multiple transcriptional regulators, several

327 ribosome- and RNA-associated factors, and the tRNA ligases LysU, TyrS, and PheS.

328 These findings indicate that MiaA is tied into a complex web of factors that can have direct

329 and indirect effects on translation. Driving this point home is the observation that MiaA

330 overexpression suppresses the production of $\operatorname{Tad} A$, an enzyme that catalyzes the

331 deamination of adenosine-to-inosine (A-to-I) in Arg2 tRNA and a select set of mRNAs

$332[79,80]$. In K-12 E. coli, the A-to-I editing function of TadA can recode at least 12 mRNAs,

333 which results in the generation of proteins with altered activities that can impact bacterial

334 cell physiology [80]. Among the known TadA-edited transcripts is one encoding IlvC, an

335 enzyme involved in isoleucine and valine biosynthesis which, like TadA, is downregulated

$336 \sim 3.5$-fold in UTI89 when MiaA is overexpressed (Fig. 7D). 


\section{UTI89 $\Delta$ miaA phenotypes are not entirely due to aberrant RpoS or Hfq expression}

340 Sigma factor RpoS $\left(\sigma^{S}\right)$ and the small RNA chaperone Hfq [7, 30, 31]. Both of these

341 factors are important for the stress resistance and virulence potential of ExPEC [62, 81].

342 In line with results from K-12 E. coli, our proteomics analysis indicated that RpoS and Hfq

343 levels were reduced 2.5- and 2.8-fold, respectively, in UTI89 $\Delta$ miaA relative to the wild-

344 type strain (Fig. 7C and Supplemental Dataset S1). RpoS downregulation in the

345 absence of miaA was also confirmed by western blot analysis (Supplemental Fig. S8A)

346 These observations suggest that the phenotypic defects associated with UTI89 $\Delta$ miaA

347 might be attributable to aberrant expression of RpoS or Hfq. However, despite some

348 similarities, the phenotypes that we previously observed with UTI89 mutants lacking

349 either rpoS or $h f q$ are distinct from one another and from those that we report here with

350 UTI89 $\triangle$ miaA $[62,81]$. Furthermore, the induced expression of recombinant RpoS or Hfq

351 (Supplemental Fig. S8B and C) failed to rescue growth of UTI89 $\Delta m i a A$ under

352 hyperosmotic conditions (Supplemental Fig. S8D and E). The pRpoS Ptac $_{\text {and }}$ pHfqptac

353 expression constructs used in these assays can complement UTI89 mutants lacking rpoS

354 or $h f q$, respectively $[62,81]$. Cumulatively, these data indicate that the phenotypes seen

355 with UTI89 $\Delta$ miaA are not entirely due to attenuated expression of either RpoS or Hfq.

356 Also of note, our ability to complement UTI89 $\Delta$ miaA with MiaA expression constructs (see

357 Figs. 2, 3, and 8) demonstrates that the phenotypic defects associated with this knockout

358 mutant are not caused by off target mutations or polar effects on hfq, which lies

359 immediately downstream of miaA. 


\section{UNN-Leu codon usage by MiaA-sensitive transcripts}

Messages like those encoded by rpoS and hfq are classified as Modification Tunable Iranscripts (MoTTs), which are identifiable by 1 ) codon usage different from that of average transcripts and 2) translation that is sensitive to changing levels of tRNA modifications [31, 82]. Studies in the K-12 E. coli strain MG1655 of rpoS, hfq, and other transcripts suggest that MiaA-sensitive MoTTs have higher than average ratios of UNNLeu codons relative to total Leu codons $[30,31]$. This led us to ask if UNN-Leu codon usage correlates with protein expression levels in UTI89 when MiaA is either absent or over-produced. Plotting results from our MudPIT analysis versus UNN-Leu codon usage (Supplemental Dataset S1) showed that just over $60 \%$ of the proteins that are differentially expressed in UTI89 $\Delta$ miaA or UTI89/pMiaAptac have UNN-Leu codon usage ratios that are greater than the K-12 average ratio of 0.22 (Fig. 9, green dashed line). In

373 line with previous findings [30, 31], RpoS and Hfq are among the differentially expressed 374 proteins with UNN-Leu codon usage ratios of greater than 0.22 . However, the average UNN-Leu codon usage ratio in UTI89 is somewhat higher than that in K-12 E. coli. Using this value, which is 0.28 , less than half of the proteins that are differentially regulated in UTI89 $\triangle$ miaA or UTI89/pMiaAptac have greater than average UNN-Leu codon usage ratios

(Fig. 9, black dashed line). Furthermore, among the proteins that are not significantly altered by either deletion or overexpression of miaA, about $30 \%$ have UNN-Leu codon

380 usage ratios greater than 0.28 . Cumulatively, these data indicate that UNN-Leu codon 381 ratios alone may not be especially useful for predicting MiaA-sensitive protein expression 382 patterns within ExPEC strains like UTI89. 


\section{DISCUSSION}

The results presented here demonstrate that MiaA is crucial for ExPEC fitness and virulence, and that changing MiaA levels can impact the translation of a broad spectrum of proteins. Our findings are in line with previously published work showing that tRNA modifying enzymes can influence the virulence potential of a variety of microbial pathogens [83]. The attenuation of bacterial virulence-related phenotypes in the absence of a specific tRNA modifying enzyme can, in some cases, be explained by sub-optimal

391 translation of specific toxins or key regulatory factors [14, 19-21]. For example, deletion 392 of miaA in the diarrheagenic bacteria Shigella flexneri ablates translation of the 393 transcriptional master regulator VirF, resulting in the reduced expression of downstream 394 virulence factors [11, 84]. Overexpression of recombinant VirF alone is sufficient to rescue 395 the miaA mutant, suggesting that low-level production of VirF is in large part responsible 396 for the virulence-related defects caused by the deletion of miaA in S. flexneri. In contrast, 397 our work indicates that the diverse phenotypes affected by MiaA expression in the ExPEC 398 isolate UTI89 are not attributable to any single factor, but rather arise due to the altered expression and dysregulation of multiple proteins and pathways downstream of MiaA. The $\mathrm{ms}^{2} \mathrm{i}^{6} \mathrm{~A}$ modification is understood to affect the fidelity of translation $[5,8,27]$.

401 Earlier work in K-12 E. coli and Salmonella strains showed that bacteria lacking miaA 402 have an increase in the +1 direction of frameshifting, but not the -1 direction $[73,74]$. In 403 these studies, the $i^{6} \mathrm{~A}$ modification was found to be a major contributor to ribosome fidelity. 404 In UTI89, significant increases in frameshifting were seen in both the +1 and -1 directions 405 when miaA was knocked out. When MiaA was overproduced, we also observed marked 406 elevation of frameshifting in the -1 direction, while frameshifting levels in the +1 direction 
407 were more modest. Most reports to date indicate that tRNA modifications typically affect 408 frameshifting primarily in one direction [5, 74]. At first glance, our data seemingly counter 409 this trend. However, we note that the expression of firefly luciferase downstream of the 410 HIV-derived linker in our reporter system may also occur as a consequence of a +2 411 frameshift, rather than a -1 frameshift, which would more closely mirror what was 412 observed in previous studies with K-12 E. coli and Salmonella strains [73, 74]. It is also 413 possible that the apparent increases in both -1 and +1 frameshifting observed in our 414 assays reflect the presence of MiaA-sensitive regulatory circuits in UTI89 that are different 415 from those in K-12 E. coli or Salmonella strains.

416 During the course of this study, we were surprised to observe that MiaA levels in 417 the ExPEC reference strain UTI89 were substantially decreased in response to high salt 418 (see Fig. 4). Ongoing work indicates that MiaA levels in UTI89 are also altered upon 419 exposure to other stressors, such as MV (Supplemental Fig. S9A and B). Due to the 420 sweeping phenotypes seen in the absence of MiaA, we hypothesized that the levels of 421 MiaA would have stayed the same or increased in response to stressors like high salt and 422 MV. However, our data indicate that MiaA levels are fine-tuned within ExPEC such that 423 too much or too little enzyme can have similarly detrimental consequences. The effects 424 of MiaA expression in our growth curve assays were dose-dependent, with high-level 425 expression of MiaA being nearly as disruptive as the deletion of miaA. For instance, low426 level expression of MiaA restored the resistance of UTI89 $\Delta$ miaA to high salt, whereas 427 overexpression of MiaA resulted in greatly increased sensitivity (see Fig. 5). MiaA is part of a complex superoperon and its regulation, and the regulation of 429 tRNA modifying enzymes in general, is not well understood $[33,85,86]$. Though we did 
430 not investigate MiaA regulation in detail here, our RT-qPCR experiments indicate that

431 MiaA levels are reduced in response to high salt stress via a post-transcriptional

432 mechanism (Fig. 4C). Interestingly, miaA has a higher-than-average UNN Leu codon

433 usage ratio of 0.46 , suggesting that MiaA may help regulate the translation of its own

434 transcripts [31]. Furthermore, we note that MiaA levels are intensified in the presence of

435 the metal chelator EDTA (Supplemental Fig. S9C), raising the possibility that the

436 quantities of this tRNA modifying enzymes are controlled by one or more EDTA-sensitive

437 metalloproteases. The factors that modulate MiaA levels during times of stress require

438 further investigation.

439 By adjusting the levels of tRNA modifying enzymes like MiaA, ExPEC and other 440 organisms may be able to vary the diversity of translated proteins and thereby optimize 441 adaptive responses to stressful stimuli [14, 33, 46, 87-90]. Indeed, the overexpression 442 and deletion of miaA led to the generation of distinct proteomes by UTI89 (Fig. 7) and 443 compromised the ability of this ExPEC strain to deal with multiple stressors. Because 444 tRNA modifications can have pleiotropic effects, it is not always easy to distinguish the 445 direct and indirect effects that tRNA modifying enzymes like MiaA have on translation 446 [90]. For example, pioneering work in K-12 E. coli indicates that the efficient translation 447 of RpoS and Hfq relies on MiaA for proper decoding of UNN-Leu codons [7, 30, 31], but 448 these factors can themselves regulate the expression of numerous other proteins [91-93].

449 The capacity for MiaA to have additional, indirect effects on the fidelity and specificity of 450 translation is further highlighted by our proteomics data showing that MiaA impacts the 451 expression of multiple ribosome- and RNA-associated factors, tRNA ligases, and the RNA 452 editing enzyme YfhC (TadA). These findings suggest the existence of a complex network 
453 of RNA and translational modifiers that can regulate the expression of one another.

454 Layered on top of this is the potential for MiaA to affect the biosynthesis and availability

455 of specific metabolites used by other tRNA modifying enzymes [15, 33].

Increases in frameshifting due to changing levels of MiaA may also allow for more

457 error-prone translation and the subsequent diversification of expressed proteins, which

458 could allow bacteria to better deal with stressful stimuli. The ability of cells to actively

459 regulate frameshifting and other translational errors in order to generate mutant proteins

460 that deviate from those encoded by the genome is gaining appreciation as an adaptive 461 response to stress [94-97]. Ongoing studies aim to utilize ribosomal profiling along with

462 RNA-seq and proteomics to determine if off-frame and mutant proteins are being 463 produced by ExPEC via translational modifiers like MiaA in response to stressful stimuli.

464 Similar lines of research may also shed light on the somewhat more cryptic functions of 465 the MiaB-catalyzed tRNA modification. In the absence of $m i a A$, the $\mathrm{i}^{6} \mathrm{~A}$ and $\mathrm{ms}^{2} \mathrm{i}^{6} \mathrm{~A}$ modifications are not detectable (Fig.

467 4), as expected from previously published work [86]. When MiaA is overproduced, high 468 levels of $\mathrm{i}^{6} \mathrm{~A}$ are observed, while $\mathrm{ms}^{2} \mathrm{i}^{6} \mathrm{~A}$ modification levels remain relatively stable (Fig.

469 5A). This suggests that only a fraction of the UNN-decoding tRNAs are fully modified in 470 the cell at any time. Interestingly, high salt stress reduces both MiaA expression and $471 \mathrm{~ms}^{2} \mathrm{i}^{6} \mathrm{~A}$ levels, but does not significantly affect i ${ }^{6} \mathrm{~A}$ levels (Fig. 4). In contrast, the disruption 472 of $m i a B$ prevents the formation of $\mathrm{ms}^{2} i^{6} \mathrm{~A}$ and causes marked increases in $\mathrm{i}^{6} \mathrm{~A}$ levels 473 (Supplemental Fig. S4), but this had no phenotypic effect in any of our assays. These 474 findings present a conundrum - why does high-level production of $\mathrm{i}^{6} \mathrm{~A}$ due to MiaA 475 overexpression attenuate the stress resistance of UTI89 while even higher levels of ${ }^{6} \mathrm{~A}$ 
476 that build up in the absence of MiaB had no overt phenotypic effects in our assays? In

477 considering this issue, it should be noted that we quantified relative levels of $\mathrm{i}^{6} \mathrm{~A}$ and

$478 \mathrm{~ms}^{2} \mathrm{i}^{6} \mathrm{~A}$, and not specific tRNAs, leaving open the possibility that changing levels of MiaA

479 differentially affect distinct tRNA subsets. This alone could account for the contrasting

480 phenotypic effects linked with elevated $i^{6} \mathrm{~A}$ levels due to MiaA overexpression versus

481 those caused by miaB deletion. MiaA overexpression may also be detrimental due to

482 depletion of substrates like dimethylallyl diphosphate (DMAPP) that feed into other critical

483 pathways, including the biosynthesis of ubiquinone. The deletion of miaB, by causing a

484 buildup of ${ }^{6} \mathrm{~A}$ rather than accelerated production of this modified residue, may have less

485 of an abrupt impact on the availability substrates like DMAPP. Finally, it is feasible that

486 MiaA has moonlighting function(s), affecting non-tRNA targets and compromising

487 bacterial fitness when produced in excess. Cumulatively, the findings presented here

488 highlight the central and complex roles that core metabolic genes like miaA can have on

489 the elaboration and fine-tuning of pathogen stress resistance and virulence-associated

490 phenotypes.

491

492 EXPERIMENTAL PROCEDURES

493 Bacterial strains. Strains used in this study are listed in Supplemental Table S1. Mutant

494 strains were constructed in the reference ExPEC isolate UTI89 using the lambda Red

495 recombination system and primers detailed in Supplemental Table S2, as previously

496 described [48]. The chloramphenicol resistance $\left(\mathrm{Clm}^{\mathrm{R}}\right)$ cassette flanked by LoxP sites

497 was amplified from plasmid pKD3 using primers that contain overhanging ends with $\sim 40$

498 bp of homology near the 5' and 3' ends of each target locus. PCR products were 
499 introduced by electroporation into UTI89 carrying pKM208, which encodes an IPTG-

500 inducible lambda Red recombinase [49]. Knockouts were verified by PCR using primers

501 indicated in Supplemental Table S2.

502

503 Plasmids. Expression and reporter constructs were generated using standard molecular

504 biology approaches and primers listed in Supplemental Table S2. The miaA and miaB 505 genes were amplified from UTI89 by PCR, digested, and ligated into pRR48 using Pst1

506 and Kpn1 restriction sites to create pMiaA $\mathrm{Ptac}_{\mathrm{P}}$ and $\mathrm{pMiaB} \mathrm{P}_{\mathrm{Ptac}}$. Sequences encoding $\mathrm{Hfq}$

507 fused with C-terminal 6xHis and Flag tags were cloned using a similar approach to make

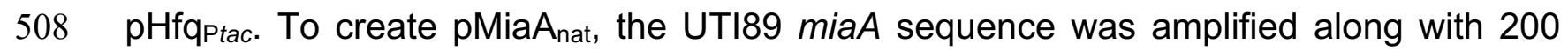

509 base pairs of flanking sequences, including the miaA promoter, and then ligated into the

510 EcoR1 site of pACYC184. The plasmid pMiaA-Flagnat, having the miaA promoter region

511 upstream of sequences encoding MiaA with a C-terminal Flag-tag, was produced 512 similarly.

513 The dual-luciferase reporter plasmids used for the frameshifting assays were 514 created using p2Luc plasmids as templates $[75,76]$. The genes encoding the renilla and

515 firefly luciferases were amplified by PCR along with intergenic Az1- or HIV-derived linker

516 sequences. A Shine-Dalgarno ribosome binding site was incorporated into the forward

517 primer (p2Luc_F) primer to promote translation of the linked luciferases. PCR products

518 were digested and ligated into the Kpnl and HindlII sites of pBAD18 $\left(\mathrm{Ap}^{\mathrm{R}}\right)$ and pBAD33

$519\left(\right.$ Cam $\left.^{R}\right)$. Plasmids with different resistance cassettes were needed for use with

520 UTI89 $\Delta$ miaA $\left(\right.$ Cam $\left.^{R}\right)$ and UTI89 carrying pMiaA ${ }_{\text {tac }}$ or the empty vector pRR48. The Az1-

521 and HIV-derived linker region sequences are noted in Fig. 6A, and were chosen because 
522 they contain MiaA-sensitive UNN codons. Control plasmids in which the Az1 and HIV

523 linkers are altered to place the two luciferases in-frame were generated in an analogous

524 fashion using previously described p2Luc plasmids as templates $[75,76]$.

525

526 Bacterial growth analysis. UTI89 and its derivatives were grown from frozen stocks in

$5275 \mathrm{ml}$ of LB, $100 \mathrm{mM}$ MES-buffered LB (MES-LB; pH 5.0), or modified M9 medium (6 g/liter

$528 \mathrm{Na}_{2} \mathrm{HPO}_{4}, 3 \mathrm{~g} / \mathrm{K} \mathrm{KH}_{2} \mathrm{PO}_{4}, 1 \mathrm{~g} / \mathrm{l} \mathrm{NH}{ }_{4} \mathrm{Cl}, 0.5 \mathrm{~g} / \mathrm{l} \mathrm{NaCl}, 1 \mathrm{mM} \mathrm{MgSO}, 0.1 \mathrm{mM} \mathrm{CaCl}, 0.1 \%$

529 glucose, $0.0025 \%$ nicotinic acid, $0.2 \%$ casein amino acids, and $16.5 \mu \mathrm{g} / \mathrm{ml}$ thiamine in

$530 \mathrm{H}_{2} \mathrm{O}$ ) at $37^{\circ} \mathrm{C}$ overnight in loosely capped 20-by-150-mm borosilicate glass tubes with

531 shaking (225 rpm, with tubes tilted at a $30^{\circ}$ angle). Overnight cultures were brought to an

$532 \mathrm{OD}_{600}$ of $\sim 1.0$ and then sub-cultured 1:100 into LB, MES-LB, or M9 medium. Growth

533 curves were acquired using a Bioscreen C instrument (Growth Curves USA) with 200- $\mu$ l

534 cultures in 100 -well honeycomb plates shaking at $37^{\circ} \mathrm{C}$. Cultures included extra $\mathrm{NaCl}(5 \%$

535 w/v), 1 mM MV (Sigma-Aldrich), 1 or 2 mM ASN (Sigma-Aldrich), or IPTG, as indicated.

536 MV and ASN solutions were prepared fresh just before use. All growth curves were

537 determined using quadruplicate samples with at least three independent replicates.

538 Overnight cultures of strains carrying plasmids for complementation experiments were

539 grown in the presence of antibiotics $(100 \mu \mathrm{g}$ of ampicillin $/ \mathrm{ml}$ or $50 \mu \mathrm{g}$ of tetracycline $/ \mathrm{ml})$

540 to maintain the plasmids, but antibiotics were not included in media used for the

541 subsequent growth assays.

543 Mouse models. All animals used in this study were handled in accordance with protocols

544 approved by the Institutional Animal Care and Use Committee at the University of Utah 
545 (Protocol number 10-02014), following US federal guidelines indicated by the Office of 546 Laboratory Animal Welfare (OLAW) and described in the Guide for the Care and Use of

547 Laboratory Animals, 8th Edition. Mice were purchased from The Jackson Laboratory, 548 housed 3 to 5 per cage, and allowed to eat (irradiated Teklad Global Soy Protein-Free 549 Extruded chow) and drink antibiotic-free water ad libitum.

$550 \quad$ Competitive gut colonization assays. For these assays, a kanamycin resistance 551 cassette $\left(\mathrm{Kan}^{\mathrm{R}}\right)$ was inserted into the attTn7 site of UTI89 to create UTI89::Kan ${ }^{\mathrm{R}}$, which 552 can be easily distinguished from the chloramphenicol resistant $\left(\mathrm{Cam}^{\mathrm{R}}\right)$ miaA and miaB 553 knockout mutants by plating on selective media. Previous work demonstrated that 554 insertion of resistance cassettes into the attTn7 site does not impact ExPEC fitness within 555 the gut $[55,56]$. Individual cultures of UTI89:: $\operatorname{Kan}^{R}$ (standing in as the wild-type strain), $556 \mathrm{UTI} 89 \mathrm{mia} A$, and UTI89 $\Delta$ miaB were grown statically from frozen stocks for $24 \mathrm{~h}$ at $37^{\circ} \mathrm{C}$ 557 in 250-ml flasks containing $20 \mathrm{ml}$ of modified M9 medium. Each knockout mutant was 558 then mixed 1:1 with UTI89::Kan ${ }^{\mathrm{R}}(6 \mathrm{ml}$ of each culture $)$ and then pelleted by centrifugation 559 at $8,000 \times g$ for 8 minutes at room temperature. The bacterial pellets were then washed 560 once with phosphate-buffered saline (PBS), pelleted again, and resuspended in $0.5 \mathrm{ml}$ of

561 PBS. Female SPF BALB/c mice aged 7 to 8 weeks were inoculated via oral gavage with $56250 \mu$ PBS containing $\sim 10^{9} \mathrm{CFU}$ of each bacterial mixture. At the indicated time points 563 post-inoculation, individual mice were placed into unused takeout boxes for a few minutes 564 for weighing and feces collection. Freshly deposited feces were collected from the boxes 565 and immediately added to $1 \mathrm{ml}$ of $0.7 \% \mathrm{NaCl}$, weighed, and set on ice. The samples were 566 then homogenized and briefly centrifuged at low speed to pellet any insoluble debris. 567 Supernatants were serially diluted and plated onto LB agar containing either 
568 chloramphenicol $(20 \mu \mathrm{g} / \mathrm{ml})$ or kanamycin $(50 \mu \mathrm{g} / \mathrm{ml})$ for selective growth of UTI89::Kan ${ }^{R}$

569 (wild type), UTI89 4 miaA, or UTI89 4 miaB. Fecal samples were also analyzed prior to the

570 start of each experiment to ensure that there were no endogenous bacteria present that

571 were resistant to chloramphenicol or kanamycin. Cls were calculated as the ratio of

572 knockout over wild-type bacteria recovered in the feces divided by the ratio of knockout

573 over wild-type bacteria present in the inoculum $[55,57]$. A total of 7 to 8 mice in two

574 independent assays were used for each set of bacterial strains tested.

UTI model. The murine UTI model was used essentially as described by our group and others [98, 99]. Wild-type UTI89 and the miaA, and miaB knockout mutants were

577 grown from frozen stocks in $20 \mathrm{ml}$ LB broth in $250 \mathrm{~mL}$ Erlenmeyer flasks without shaking

578 at $37^{\circ} \mathrm{C}$ for 24 hours. Bacteria were then pelleted by centrifugation ( 8 minutes at $8,000 x$

579 g) and resuspended in PBS. Seven- to eight-week-old female CBA/J or C3H/HeJ mice

580 were briefly anesthetized by isoflurane inhalation and slowly inoculated via transurethral

581 catheterization with $50 \mu \mathrm{L}$ of PBS containing a suspension of $\sim 10^{7}$ bacteria. Bacterial

582 reflux into the kidneys using this procedure is rare, occurring in less than $1 \%$ of the test

583 animals. At $0.25,1,3$, or 9 days post-inoculation, mice were sacrificed and bladders were

584 harvested aseptically, weighed, and homogenized in $1 \mathrm{ml}$ PBS containing $0.025 \%$ Triton

$585 \mathrm{X}-100$. Bacterial titers within the homogenates were determined by plating serial dilutions

586 on LB agar plates. Nine or more mice in total, from two independent experiments, were

587 used for each bacterial strain and time point examined.

Sepsis model. UTI89, UTI89 $\Delta$ miaA, and UTI89 $\Delta$ miaB were grown from frozen

589 stocks in $20 \mathrm{ml} \mathrm{M9}$ broth without shaking at $37^{\circ} \mathrm{C}$ for $24 \mathrm{~h}$, pelleted by centrifugation at

$5908,000 \times g$ for 8 minutes, and washed once with PBS, pelleted again, and resuspended in 
591 PBS. Seven- to eight-week-old female C57BI/6 mice were briefly anesthetized by

592 isoflurane inhalation and infected via intraperitoneal injection of $\sim 10^{7}$ CFU within $200 \mu$ l

593 PBS. Mice were monitored over a 72-hour period for signs of morbidity and mortality.

594 Alternatively, at 6 hours post-inoculation mice were sacrificed and the liver, kidneys, and

595 spleens were harvested aseptically, weighed, and homogenized in $1 \mathrm{ml}$ PBS containing

$596 \quad 0.025 \%$ Triton X-100. Bacterial titers within the homogenates were determined by plating

597 serial dilutions on LB agar plates.

Invasion, adhesion, and intracellular persistence assays. Bacteria were grown at

$60037^{\circ} \mathrm{C}$ for $48 \mathrm{~h}$ in $20 \mathrm{~mL}$ static LB broth to induce expression of type 1 pili, which are

601 important mediators of UPEC adherence and entry into host cells [99]. Host cell

602 association and gentamicin protection-based invasion and overnight intracellular

603 persistence assays were performed as previously described using the human bladder

604 epithelial cell line 5637 (HTB-9; ATCC) [100]. Of note, UTI89 $\Delta$ miaA is about 3-fold more

605 sensitive to the host-cell impermeable antibiotic gentamicin, as determined by using

606 Etest Strips (VWR) (Supplemental Fig. S10). However, this likely had no effect on

607 results from the cell culture-based invasion and intracellular survival experiments, as the

608 concentrations of gentamicin (100 and $10 \mu \mathrm{g} / \mathrm{ml})$ used in these assays exceed those

609 needed to effectively kill extracellular UTI89, UTI89 $\Delta$ miaB, and UTI89 $\Delta$ miaA.

611 Biofilm analysis. In vitro rugose biofilm assays were performed starting with cultures 612 grown overnight at $37^{\circ} \mathrm{C}$ shaking in LB, as described [78]. Bacteria from each culture 613 were then brought to an $\mathrm{OD}_{600}$ of $\sim 1.0$ and $10 \mu$ aliquots were spotted onto YESCA agar 
614 plates (12 g/l Casamino acids, $1.2 \mathrm{~g}$ yeast extract, $22 \mathrm{~g}$ agar) and incubated at RT ( 20-

$61522^{\circ} \mathrm{C}$ ). After 14 days, biofilm images were acquired by focus stacking using an M.Zuiko

616 Digital ED $60 \mathrm{~mm}$ lens mounted on an Olympus OM-D E-M1 Mark II camera.

617

618 Motility assays. Cultures of UTI89, UTI89 $\Delta m i a A$, and UTI89 $\Delta$ miaB grown overnight

619 shaking in LB or M9 medium were brought to $\mathrm{OD}_{600}$ of 1.0. Swim motility plates, containing

$620 \quad 0.2 \%$ agar in LB or M9 medium, were inoculated with $2 \mu$ l of each bacterial suspension

621 delivered just below the agar surface. The diameter of bacterial spreading was measured

622 every 1-2 hours over the course of an $8-10$ hour-incubation at $37^{\circ} \mathrm{C}$. Swim rates were

623 calculated during logarithmic growth. To assess the effects of MiaA and MiaB

624 overexpression on motility, tryptone soft agar plates [101] containing $50 \mu \mathrm{g} / \mathrm{ml}$ ampicillin

625 and $100 \mu \mathrm{M}$ IPTG were inoculated with UTI89/pRR48, UTI89/pMiaAPtac, and

626 UTI89/pMiaB ${ }_{\text {Ptac }}$ from overnight shaking cultures. Plates were imaged after a 6-hour

627 incubation at $37^{\circ} \mathrm{C}$.

628

629 Acid resistance assays. Bacterial strains from overnight cultures were diluted 1:100 in

630 fresh LB and grown shaking at $37^{\circ} \mathrm{C}$ for $3 \mathrm{~h}$. Concentrated $\mathrm{HCl}$ was then added to each

631 culture to adjust the $\mathrm{pH}$ to 3.0 and incubations were continued for another 30 minutes.

632 Bacteria from $1 \mathrm{ml}$ of each culture were then pelleted at $16,000 \times \mathrm{g}$ for 5 min and washed

633 in PBS. Surviving bacteria were enumerated by plating serial dilutions on LB agar and 634 normalized to input titers. 
636 Osmotic stress resistance assays. UTI89/pACYC184, UTI89

637 UTI89 $\triangle$ miaA/pMiaAnat, and UTI89 $\Delta$ miaB were grown shaking overnight at $37^{\circ} \mathrm{C}$ in $5 \mathrm{ml}$

638 LB broth with $20 \mu \mathrm{g} / \mathrm{ml}$ tetracycline and then back diluted 1:100 into $5 \mathrm{ml}$ fresh LB (+

639 tetracycline). After $5 \mathrm{~h}$ shaking at $37^{\circ} \mathrm{C}$, a $1-\mathrm{ml}$ aliquot of each culture was pelleted,

640 resuspended in $1 \mathrm{ml}$ of sterile water with or without $0.1 \%$ glucose, and incubations were

641 continued for another $2 \mathrm{~h}$ with shaking at $37^{\circ} \mathrm{C}$. Viable bacteria present at $0,30,60,90$,

642 and 120 min after resuspension in water were quantified by dilution plating and

643 normalized to input titers. Growth curves in $\mathrm{LB} \pm 5 \% \mathrm{NaCl}$ were acquired as described

644 above.

Western blot analysis. Bacterial pellets were frozen at $-80^{\circ} \mathrm{C}$ and then resuspended in

B-PER lysis reagent (Thermo Scientific) supplemented with $1 \mathrm{mM}$ phenylmethylsulfonyl

648 fluoride, protease inhibitor cocktail (Roche), and Lysonase Bioprocessing Reagent 649 (Novagen). After a 15-minute incubation at room temperature, samples were spun for 1

650 minute at $13,000 \times g$ to remove large cell debris, and protein concentrations in the 651 supernatants were determined using the BCA reagent system (Pierce). Equivalent protein

652 amounts were resolved by SDS-PAGE and subsequently transferred to Immobilon PVDF-

653 FL membranes (Millipore). Blots were probed using mouse anti-Flag M2 (1:3000; Sigma-

654 Aldrich), rabbit anti-Flag (Immunology Consultants laboratory, inc.), and mouse anti-

655 RpoS (anti-SigmaS; Biolegend) and visualized using enhanced chemiluminescence with

656 HRP-conjugated secondary antibodies (1:3000 or 1:5000; Amersham Biosciences), as

657 described [102]. To ensure that equivalent amounts of protein from each sample were

658 analyzed, blots were re-probed using rabbit anti-E. coli antisera (1:2,000 or 1:5000;

659 BioDesign International). 
Analysis of relative i6A and ms2i6A levels. UTI89 and UTI89 $\Delta m i a A$ were grown from

662 frozen stocks shaking at $37^{\circ} \mathrm{C}$ overnight in LB. UTI89/pRR48 and UTI89/pMiaA $A_{\text {tac }}$ were

663 grown similarly using LB supplemented with ampicillin $(100 \mu \mathrm{g} / \mathrm{ml})$. The bacteria were

664 sub-cultured 1:100 into $6 \mathrm{ml}$ of LB $\pm 1 \mathrm{mM}$ IPTG and then grown shaking to an OD 600 of

665 0.5. After adjusting the cultures to $O D_{600}$ of 1.0 , the bacteria were pelleted by spinning

666 at $8000 \times g$ for 1.5 minutes. Pellets were then resuspended in $1 \mathrm{ml}$ of RNAlater

667 Stabilization Solution (ThermoFisher) and stored at $4^{\circ} \mathrm{C}$ overnight prior to extraction of

668 RNA using a Norgen Total RNA Extraction Kit.

Samples were analyzed using a Hypersil GOLD C18 column $(2.1 \mathrm{~mm} \times 150 \mathrm{~mm}$,

$6701.9 \mu \mathrm{m}$ particle size; Thermo Fisher) attached to a Thermo Scientific Dionex UltiMate

6713000 UHPLC instrument in line with an LTQ-OrbiTrap XL instrument (Thermo Fisher).

672 The LC-MS parameters were based upon a procedure described previously [103, 104],

673 with the following adjustments. The UHPLC column was pre-equilibrated in $100 \%$ Buffer

674 A [50 mM ammonium acetate (Fisher) in LC-MS Optima water]. Buffer B consisted of

$67560 \%$ (v/v) LC-MS Optima acetonitrile (Fisher) and 40\% LC-MS water (Fisher). The

676 reaction components were eluted at a rate of $0.2 \mathrm{ml} / \mathrm{minute}$ with the following program:

$6770 \% \mathrm{~B}$ from 0 to $3.46 \mathrm{~min}, 0$ to $0.9 \% \mathrm{~B}$ from 3.46 to $3.69 \mathrm{~min}, 0.9$ to $1.5 \% \mathrm{~B}$ from 3.69 to

$6783.92 \mathrm{~min}, 1.5$ to $3 \%$ B from 3.92 to $4.25 \mathrm{~min}, 3$ to $20 \%$ B from 4.25 to $6.5 \mathrm{~min}, 20$ to $25 \%$

679 B from 6.5 to $7 \mathrm{~min}, 25$ to $40 \%$ B from 7 to $8.5 \mathrm{~min}, 40$ to $45 \%$ B from 8.5 to $9.25 \mathrm{~min}$,

68045 to $60 \%$ B from 9.25 to $9.95 \mathrm{~min}, 60$ to $100 \%$ B from 9.95 to $10.45 \mathrm{~min}, 100 \%$ B from

68110.45 to $16 \mathrm{~min}, 100$ to $0 \% \mathrm{~B}$ from 16 to $16.1 \mathrm{~min}$, and $0 \% \mathrm{~B}$ from 16.1 to $20 \mathrm{~min}$. The 
682 flow from the column was diverted to the mass spectrometer from 3.5 minutes to 17

683 minutes during the UHPLC program. The mass spectrometer was operated in positive

684 ion mode, and authentic guanosine material (Sigma Aldrich) was used to generate a

685 tune file for the instrument. The observed $\mathrm{m} / \mathrm{z}$ values of the +1 charge states of the i6a

686 and ms2i6a RNA bases were 336.1658 and 382.1535, respectively. The observed

687 retention times for $\mathrm{i} 6 \mathrm{~A}$ and $\mathrm{ms} 2 \mathrm{i} 6 \mathrm{a}$ were determined from the center of their extracted

688 ion chromatogram peaks to be 15.55 and 16.45 minutes, respectively. The retention

689 time of i6a from biological extracts was consistent with the retention time of authentic

690 i6a material (Cayman Chemical). Absolute intensities of the i6a ions were retrieved from

691 the mass spectrum scanned between $15.4-16.1$ minutes, while the absolute intensities

692 of the ms2i6a ions were retrieved from the mass spectrum scanned between 16.2 and

69317.0 minutes. The scan range was chosen to include the entire peak of an EIC trace,

694 excluding mass spectral data recorded out of these bounds. This ensured that the

695 intensities of ions 336.17 and 382.15 arise from eluted $\mathrm{i}^{6} \mathrm{~A}$ and $\mathrm{ms}^{2} \mathrm{i}^{6} \mathrm{~A}$ material and did

696 not include background during the rest of the run. The scan windows were wide enough

697 to account for any small drift in retention that might occur from sample to sample.

698 Because total RNA concentration varied by sample, the samples were normalized

699 against the total RNA concentration of each sample, as estimated via a NanoDrop

700 measurements at $260 \mathrm{~nm}$.

701 Quantification of frameshifting. UTI89, UTI89 $\Delta m i a A$, UTI89/pRR48 and

702 UTI89/pMiaA ${ }_{\text {Ptac }}$ carrying one of the dual-luciferase reporter plasmids (see

703 Supplemental Table S1) were grown overnight in LB supplemented with

704 chloramphenicol $(20 \mu \mathrm{g} / \mathrm{ml})$ or ampicillin $(100 \mu \mathrm{g} / \mathrm{ml})$. The cells were sub-cultured 1:100 
705 into $6 \mathrm{ml}$ LB with and without $1 \mathrm{mM}$ IPTG. At an $\mathrm{OD}_{600}$ of 0.2 , arabinose $(0.2 \%)$ was

706 added to all of the cultures to induce expression of the luciferases. Cells were allowed

707 to continue growing until reaching an $\mathrm{OD}_{600}$ of 0.5 , at which point the cultures were

708 adjusted to an $\mathrm{OD}_{600}$ of 1.0 and pelleted by spinning at $8000 \times g$ for 1.5 minutes. The

709 pellets were then subjected to one freeze-thaw cycle before being resuspended in

710 Passive Lysis Buffer (Promega; E1910). One scoop of $0.15 \mathrm{~mm}$ zirconium oxide beads

711 (Next Advance; ZrOB015) was added to each tube and bacteria were lysed using a

712 Bullet Blender (Next Advance) set at speed 8 for 3 min. After a 30-second spin in a

713 microfuge to pellet beads and any large debris, the supernatants were collected and

714 luciferase activities were analyzed as previously described [76]. Briefly, the Dual-

715 Luciferase Reporter Assay System (Promega) was used in combination with the Veritas

716 Microplate Luminometer from Turner Biosystems to quantify activity of the two

717 luciferases. Frameshifting was calculated by first determining the ratio of firefly to renilla

718 luciferase activity for each sample, and then normalizing each out-of-frame construct

719 (pCWR43 and pCWR45) with their associated in-frame control (pCWR42 and pCWR44, 720 respectively).

722 Proteomics. UTI89, UTI89 $\Delta$ miaA, UTI89/pRR48, and UTI89/pMiaAptac were grown to 723 mid-log phase $\left(\mathrm{OD}_{600} \sim 0.5\right)$ in $\mathrm{LB}$ shaking at $37^{\circ} \mathrm{C}$. IPTG (1 mM) was included for

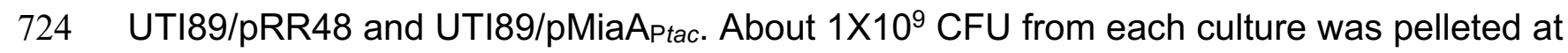
$7258,000 \times g$ for 1.5 minutes. Supernatants were then removed and cells were plunged into 726 liquid nitrogen and subsequently analyzed using MudPIT with the MSRC Proteomics Core 727 at Vanderbilt University. Label-free quantification (LFQ) values were loaded into Prostar 
728 software for statistical analysis and visualization. The data set was filtered by requiring all

729 conditions to contain at least two values. Imputation for partially observed values was

730 done with the Structured Least Square Adaptative algorithm. Imputation for conditions in

731 which values were missing for a specific protein in all three biological replicates used the

732 DetQuantile algorithm with the settings Quantile:2.5 and Factor:1. Statistical analysis was

733 performed using the 1vs1 settings and Student's $t$-tests. Differentially expressed proteins

734 were categorized (Supplemental Dataset S1) based on literature searches and

735 information drawn from EcoCyc ([105]; http://ecocyc.org/), STRING Protein-Protein

736 Interaction Networks Functional Enrichment Analysis ([106]; https://string-db.org/), and

737 Phyre2 ([107]; http://www.sbg.bio.ic.ac.uk/ phyre2/html/page.cgi?id=index). The

738 proteomics output files will be uploaded to ProteomeXchange.

740 RT-qPCR analysis. UTI89 was diluted 1:100 from overnight cultures into fresh LB, grown

741 shaking for 2.5 hours at $37^{\circ} \mathrm{C}$ prior to resuspension in LB or LB $+5 \% \mathrm{NaCl}$. After another

742 one-hour incubation bacteria were pelleted and total RNA was extracted using the

743 miRNeasy mini kit (QIAGEN). RNA samples were treated with RNase-Free DNase

744 (QIAGEN) and cDNA was made using SuperScript IV VILO Master Mix (Invitrogen)

745 according to the manufacturer's protocol. Quantitative PCR (qPCR) was carried out using

746 primers listed in Supplemental Table $\mathbf{S 2}$ with the PowerUp SYBR Green Master Mix

747 (Thermo Fisher Scientific) on a QuantStudio 3 Real-Time PCR Instrument (Applied

748 Biosystems). Replicas were made for each cDNA sample and miaA and miaB levels

749 were normalized to $r p o D$. Products were resolved in $1.5 \%$ agarose gels, stained with 
750 ethidium bromide, and visualized using a GelDoc system (BioRad Technologies) to help

751 verify the specificity of the RT-qPCR results.

Codon usage analysis. Codon frequencies (UNN Stats tab, Supplemental Dataset S1)

were calculated for each gene in UTI89 (accessions CP000243.1 and CP000244.1) using custom Python scripts that leverage the BioPython and NumPy packages.

Statistical analysis. P values were determined as indicated by Log-Rank (Mantel-Cox),

Mann-Whitney $U$ tests, ANOVA, or Student's t-tests performed using Prism 9.0.0 software, with corrections as indicated (GraphPad Software). Data distribution normality (Gaussian) was not assumed, such that non-parametric tests were used for the mouse experiments. $P$-values of less than or equal to 0.05 were defined as significant.

\section{ACKNOWLEDGEMENTS}

We thank W. Hayes McDonald of the Vanderbilt School of Medicine Mass Spectrometry Research Center for help with the proteomics. This study was funded in part by NIH grants to M.A.M. (GM134331, Al135918, Al095647, and Al088086) and to V.B. (GM126956). M.G.B. was supported by T32 Al055434 from the National Institute of Allergy and

768 Infectious Diseases, and A.J.L. was supported by T32 DK007115 from the National 769 Institute of Diabetes and Digestive and Kidney Diseases. The authors have no conflicts 770 of interest to declare. 


\section{Author Contributions}

774

775

776

777

778

779

780

781

782

783

784

785

786

787

788

789

790

MGB, BAF, and MAM designed, supervised the study, performed research, analyzed the data, and drafted the paper. WMK, AJL, JRB, MH, VB, and MTH helped with design, experimentation, and editing. AT, CJB, LMB, and QZ performed experiments and helped process samples. All authors contributed to and approved the submission of this paper.

\section{REFERENCES}

1. Jackman JE, Alfonzo JD. Transfer RNA modifications: nature's combinatorial chemistry playground. Wiley Interdiscip Rev RNA. 2013;4(1):35-48. doi:

10.1002/wrna.1144. PubMed PMID: 23139145; PubMed Central PMCID: PMCPMC3680101.

2. Bjork GR, Durand JM, Hagervall TG, Leipuviene R, Lundgren HK, Nilsson K, et al. Transfer RNA modification: influence on translational frameshifting and metabolism. FEBS Lett. 1999;452(1-2):47-51. Epub 1999/06/22. PubMed PMID: 10376676. 3. Gustilo EM, Vendeix FA, Agris PF. tRNA's modifications bring order to gene expression. Curr Opin Microbiol. 2008;11(2):134-40. doi: 10.1016/j.mib.2008.02.003. PubMed PMID: 18378185; PubMed Central PMCID: PMCPMC2408636.

4. Boccaletto P, Machnicka MA, Purta E, Piatkowski P, Baginski B, Wirecki TK, et al. MODOMICS: a database of RNA modification pathways. 2017 update. Nucleic Acids Research. 2018;46(D1):D303-d7. Epub 2017/11/07. doi: 10.1093/nar/gkx1030. PubMed PMID: $29106616 ;$ PubMed Central PMCID: PMCPMC5753262.

5. Bjork GR, Hagervall TG. Transfer RNA Modification: Presence, Synthesis, and Function. EcoSal Plus. 2014;6(1). doi: 10.1128/ecosalplus.ESP-0007-2013. PubMed PMID: 26442937.

6. Blum PH. Reduced leu operon expression in a miaA mutant of Salmonella typhimurium. J Bacteriol. 1988;170(11):5125-33. Epub 1988/11/01. PubMed PMID: 3141379; PubMed Central PMCID: PMC211580.

7. Thompson KM, Gottesman S. The MiaA tRNA modification enzyme is necessary for robust RpoS expression in Escherichia coli. J Bacteriol. 2014;196(4):754-61. doi: 
10.1128/JB.01013-13. PubMed PMID: 24296670; PubMed Central PMCID:

803 PMCPMC3911166.

804 8. Schweizer U, Bohleber S, Fradejas-Villar N. The modified base

805 isopentenyladenosine and its derivatives in tRNA. RNA biology. 2017;14(9):1197-208.

806 Epub 2017/03/10. doi: 10.1080/15476286.2017.1294309. PubMed PMID: 28277934;

807 PubMed Central PMCID: PMCPMC5699536.

808 9. Ahn KS, Ha U, Jia J, Wu D, Jin S. The truA gene of Pseudomonas aeruginosa is 809 required for the expression of type III secretory genes. Microbiology. 2004;150(Pt

810 3):539-47. Epub 2004/03/03. PubMed PMID: 14993303.

811 10. Shippy DC, Fadl AA. tRNA modification enzymes GidA and MnmE: potential role

812 in virulence of bacterial pathogens. Int J Mol Sci. 2014;15(10):18267-80. doi:

813 10.3390/ijms151018267. PubMed PMID: 25310651; PubMed Central PMCID:

814 PMCPMC4227215.

815 11. Durand JM, Dagberg B, Uhlin BE, Bjork GR. Transfer RNA modification,

816 temperature and DNA superhelicity have a common target in the regulatory network of

817 the virulence of Shigella flexneri: the expression of the virF gene. Mol Microbiol.

818 2000;35(4):924-35. Epub 2000/02/26. PubMed PMID: 10692168.

819 12. Chaudhuri RR, Peters SE, Pleasance SJ, Northen H, Willers C, Paterson GK, et

820 al. Comprehensive identification of Salmonella enterica serovar Typhimurium genes

821 required for infection of BALB/c mice. PLoS Pathog. 2009;5(7):e1000529. doi:

822 10.1371/journal.ppat.1000529. PubMed PMID: 19649318; PubMed Central PMCID:

823 PMCPMC2712085.

824 13. Gray J, Wang J, Gelvin SB. Mutation of the miaA gene of Agrobacterium

825 tumefaciens results in reduced vir gene expression. J Bacteriol. 1992;174(4):1086-98.

826 Epub 1992/02/01. PubMed PMID: 1735704; PubMed Central PMCID: PMC206401.

827 14. Chionh YH, McBee M, Babu IR, Hia F, Lin W, Zhao W, et al. tRNA-mediated

828 codon-biased translation in mycobacterial hypoxic persistence. Nature Comm.

829 2016;7:13302. Epub 2016/11/12. doi: 10.1038/ncomms13302. PubMed PMID:

$83027834374 ;$ PubMed Central PMCID: PMCPMC5114619.

831 15. Koshla O, Yushchuk O, Ostash I, Dacyuk Y, Myronovskyi M, Jager G, et al.

832 Gene miaA for post-transcriptional modification of tRNAXXA is important for 
833 morphological and metabolic differentiation in Streptomyces. Mol Microbiol.

834 2019;112(1):249-65. Epub 2019/04/25. doi: 10.1111/mmi.14266. PubMed PMID:

83531017319.

836 16. Thongdee N, Jaroensuk J, Atichartpongkul S, Chittrakanwong J, Chooyoung K,

837 Srimahaeak T, et al. TrmB, a tRNA m7G46 methyltransferase, plays a role in hydrogen

838 peroxide resistance and positively modulates the translation of katA and katB mRNAs in

839 Pseudomonas aeruginosa. Nucleic acids research. 2019;47(17):9271-81. Epub

840 2019/08/21. doi: 10.1093/nar/gkz702. PubMed PMID: 31428787; PubMed Central

841 PMCID: PMCPMC6755087.

842 17. Gao T, Tan M, Liu W, Zhang C, Zhang T, Zheng L, et al. GidA, a tRNA

843 Modification Enzyme, Contributes to the Growth, and Virulence of Streptococcus suis

844 Serotype 2. Front Cell Infect Microbiol. 2016;6:44. Epub 2016/05/06. doi:

845 10.3389/fcimb.2016.00044. PubMed PMID: 27148493; PubMed Central PMCID:

846 PMCPMC4835480.

847 18. Li D, Shibata Y, Takeshita T, Yamashita Y. A novel gene involved in the survival

848 of Streptococcus mutans under stress conditions. Applied and Environmental

849 Microbiology. 2014;80(1):97-103. Epub 2013/10/15. doi: 10.1128/AEM.02549-13.

850 PubMed PMID: 24123744; PubMed Central PMCID: PMCPMC3910998.

851 19. Cho $\mathrm{KH}$, Caparon MG. tRNA modification by GidA/MnmE is necessary for

852 Streptococcus pyogenes virulence: a new strategy to make live attenuated strains.

853 Infection and Immunity. 2008;76(7):3176-86. Epub 2008/04/23. doi: 10.1128/IAI.01721-

854 07. PubMed PMID: 18426891; PubMed Central PMCID: PMCPMC2446735.

855 20. Kinscherf TG, Willis DK. Global regulation by gidA in Pseudomonas syringae. J

856 Bacteriol. 2002;184(8):2281-6. Epub 2002/03/27. doi: 10.1128/jb.184.8.2281-

857 2286.2002. PubMed PMID: 11914360; PubMed Central PMCID: PMCPMC134964.

858 21. Sha J, Kozlova EV, Fadl AA, Olano JP, Houston CW, Peterson JW, et al.

859 Molecular characterization of a glucose-inhibited division gene, gidA, that regulates

860 cytotoxic enterotoxin of Aeromonas hydrophila. Infection and immunity.

861 2004;72(2):1084-95. Epub 2004/01/27. doi: 10.1128/iai.72.2.1084-1095.2004. PubMed

862 PMID: 14742556; PubMed Central PMCID: PMCPMC321642. 
863 22. Kaminska KH, Baraniak U, Boniecki M, Nowaczyk K, Czerwoniec A, Bujnicki JM.

864 Structural bioinformatics analysis of enzymes involved in the biosynthesis pathway of

865 the hypermodified nucleoside ms(2)io(6)A37 in tRNA. Proteins. 2008;70(1):1-18. Epub

866 2007/10/03. doi: 10.1002/prot.21640. PubMed PMID: 17910062.

867 23. Persson BC. Modification of tRNA as a regulatory device. Mol Microbiol.

868 1993;8(6):1011-6. Epub 1993/06/01. PubMed PMID: 7689685.

869 24. Caillet J, Droogmans L. Molecular cloning of the Escherichia coli miaA gene

870 involved in the formation of delta 2-isopentenyl adenosine in tRNA. J Bacteriol.

871 1988;170(9):4147-52. Epub 1988/09/01. PubMed PMID: 3045085; PubMed Central

872 PMCID: PMC211421.

873 25. Seif E, Hallberg BM. RNA-protein mutually induced fit: structure of Escherichia

874 coli isopentenyl-tRNA transferase in complex with tRNA(Phe). J Biol Chem.

875 2009;284(11):6600-4. Epub 2009/01/23. doi: 10.1074/jbc.C800235200. PubMed PMID:

876 19158097; PubMed Central PMCID: PMC2652265.

877 26. Pierrel F, Douki T, Fontecave M, Atta M. MiaB protein is a bifunctional radical-S-

878 adenosylmethionine enzyme involved in thiolation and methylation of tRNA. J Biol

879 Chem. 2004;279(46):47555-63. Epub 2004/09/02. doi: 10.1074/jbc.M408562200.

880 PubMed PMID: 15339930.

881 27. Ericson JU, Bjork GR. Pleiotropic effects induced by modification deficiency next

882 to the anticodon of tRNA from Salmonella typhimurium LT2. J Bacteriol.

883 1986;166(3):1013-21. Epub 1986/06/01. PubMed PMID: 2423501; PubMed Central

884 PMCID: PMC215226.

885 28. Eisenberg SP, Yarus M, Soll L. The effect of an Escherichia coli regulatory

886 mutation on transfer RNA structure. J Mol Biol. 1979;135(1):111-26. PubMed PMID:

88793644.

888 29. Gowrishankar J, Pittard J. Regulation of phenylalanine biosynthesis in

889 Escherichia coli K-12: control of transcription of the pheA operon. J Bacteriol.

890 1982;150(3):1130-7. PubMed PMID: 7042684; PubMed Central PMCID:

891 PMCPMC216333.

892 30. Aubee JI, Olu M, Thompson KM. TrmL and TusA Are Necessary for rpoS and

893 MiaA Is Required for hfq Expression in Escherichia coli. Biomolecules. 2017;7(2). Epub 
894 2017/05/05. doi: 10.3390/biom7020039. PubMed PMID: 28471404; PubMed Central 895 PMCID: PMCPMC5485728.

896 31. Aubee JI, Olu M, Thompson KM. The i6A37 tRNA modification is essential for 897 proper decoding of UUX-Leucine codons during rpoS and iraP translation. RNA (New 898 York, NY). 2016;22(5):729-42. Epub 2016/03/17. doi: 10.1261/rna.053165.115. PubMed 899 PMID: 26979278; PubMed Central PMCID: PMCPMC4836647.

900 32. Krasich R, Wu SY, Kuo HK, Kreuzer KN. Functions that protect Escherichia coli 901 from DNA-protein crosslinks. DNA Repair (Amst). 2015;28:48-59. doi:

902 10.1016/j.dnarep.2015.01.016. PubMed PMID: 25731940; PubMed Central PMCID: 903 PMCPMC4385401.

904 33. Connolly DM, Winkler ME. Genetic and physiological relationships among the 905 miaA gene, 2-methylthio-N6-(delta 2-isopentenyl)-adenosine tRNA modification, and 906 spontaneous mutagenesis in Escherichia coli K-12. J Bacteriol. 1989;171(6):3233-46.

907 Epub 1989/06/01. PubMed PMID: 2656644; PubMed Central PMCID: PMC210042.

908 34. Yanofsky C. Mutations affecting tRNATrp and its charging and their effect on

909 regulation of transcription termination at the attenuator of the tryptophan operon.

910 Journal of Molecular Biology. 1977;113(4):663-77. Epub 1977/07/15. PubMed PMID:

911330867.

912 35. Mikkola R, Kurlan CG. Media dependence of translational mutant phenotype.

913 FEMS Microbiol Lett 1988;56(3):265-9. doi: DOI: http://dx.doi.org/10.1111/j.1574-

914 6968.1988.tb03189.x.

915 36. Forouhar F, Arragain S, Atta M, Gambarelli S, Mouesca JM, Hussain M, et al.

916 Two Fe-S clusters catalyze sulfur insertion by radical-SAM methylthiotransferases. Nat

917 Chem Biol. 2013;9(5):333-8. doi: 10.1038/nchembio.1229. PubMed PMID: 23542644;

918 PubMed Central PMCID: PMCPMC4118475.

919 37. Leung HC, Chen Y, Winkler ME. Regulation of substrate recognition by the MiaA 920 tRNA prenyltransferase modification enzyme of Escherichia coli K-12. J Biol Chem.

921 1997;272(20):13073-83. Epub 1997/05/16. PubMed PMID: 9148919.

922 38. Denamur E, Clermont O, Bonacorsi S, Gordon D. The population genetics of 923 pathogenic Escherichia coli. Nat Rev Microbiol. 2021;19(1):37-54. Epub 2020/08/23.

924 doi: 10.1038/s41579-020-0416-x. PubMed PMID: 32826992. 
925 39. Vila J, Saez-Lopez E, Johnson JR, Romling U, Dobrindt U, Canton R, et al.

926 Escherichia coli: an old friend with new tidings. FEMS microbiology reviews.

927 2016;40(4):437-63. Epub 2017/02/16. doi: 10.1093/femsre/fuw005. PubMed PMID:

92828201713.

929 40. Barber AE, Norton JP, Spivak AM, Mulvey MA. Urinary tract infections: current

930 and emerging management strategies. Clin Infect Dis. 2013;57(5):719-24. Epub

931 2013/05/07. doi: 10.1093/cid/cit284. PubMed PMID: 23645845; PubMed Central

932 PMCID: PMC3739462.

933 41. Hunstad DA, Justice SS. Intracellular lifestyles and immune evasion strategies of

934 uropathogenic Escherichia coli. Annu Rev Microbiol. 2010;64:203-21. Epub 2010/09/10.

935 doi: 10.1146/annurev.micro.112408.134258. PubMed PMID: 20825346.

936 42. Schwab S, Jobin K, Kurts C. Urinary tract infection: recent insight into the

937 evolutionary arms race between uropathogenic Escherichia coli and our immune

938 system. Nephrology, Dialysis, Transplantation: official publication of the European

939 Dialysis and Transplant Association - European Renal Association. 2017;32(12):1977-

940 83. Epub 2017/03/25. doi: 10.1093/ndt/gfx022. PubMed PMID: 28340252.

941 43. O'Brien VP, Hannan TJ, Schaeffer AJ, Hultgren SJ. Are you experienced?

942 Understanding bladder innate immunity in the context of recurrent urinary tract infection.

943 Curr Opin Infect Dis. 2015;28(1):97-105. doi: 10.1097/QCO.0000000000000130.

944 PubMed PMID: 25517222; PubMed Central PMCID: PMCPMC4365976.

945 44. Lacerda Mariano L, Ingersoll MA. The immune response to infection in the

946 bladder. Nat Rev Urol. 2020;17(8):439-58. Epub 2020/07/15. doi: 10.1038/s41585-020-

947 0350-8. PubMed PMID: 32661333.

948 45. Svensson L, Poljakovic M, Demirel I, Sahlberg C, Persson K. Host-Derived Nitric

949 Oxide and Its Antibacterial Effects in the Urinary Tract. Adv Microb Physiol. 2018;73:1-

950 62. Epub 2018/09/29. doi: 10.1016/bs.ampbs.2018.05.001. PubMed PMID: 30262107.

951 46. Chan CT, Dyavaiah M, DeMott MS, Taghizadeh K, Dedon PC, Begley TJ. A

952 quantitative systems approach reveals dynamic control of tRNA modifications during

953 cellular stress. PLoS Genet. 2010;6(12):e1001247. Epub 2010/12/29. doi:

954 10.1371/journal.pgen.1001247. PubMed PMID: 21187895; PubMed Central PMCID:

955 PMC3002981. 
956 47. Barber AE, Norton JP, Wiles TJ, Mulvey MA. Strengths and Limitations of Model

957 Systems for the Study of Urinary Tract Infections and Related Pathologies. Microbiology

958 and Molecular Biology Reviews: MMBR. 2016;80(2):351-67. Epub 2016/03/05. doi:

959 10.1128/MMBR.00067-15. PubMed PMID: 26935136; PubMed Central PMCID:

960 PMCPMC4867371.

961 48. Datsenko KA, Wanner BL. One-step inactivation of chromosomal genes in

962 Escherichia coli K-12 using PCR products. Proc Natl Acad Sci U S A.

963 2000;97(12):6640-5. PubMed PMID: 10829079.

964 49. Murphy KC, Campellone KG. Lambda Red-mediated recombinogenic

965 engineering of enterohemorrhagic and enteropathogenic E. coli. BMC Mol Biol.

966 2003;4:11. PubMed PMID: 14672541.

967 50. Forde BM, Roberts LW, Phan MD, Peters KM, Fleming BA, Russell CW, et al.

968 Population dynamics of an Escherichia coli ST131 lineage during recurrent urinary tract

969 infection. Nature Comm. 2019;10(1):3643. Epub 2019/08/15. doi: 10.1038/s41467-019-

970 11571-5. PubMed PMID: 31409795; PubMed Central PMCID: PMCPMC6692316.

971 51. Moreno E, Andreu A, Pigrau C, Kuskowski MA, Johnson JR, Prats G.

972 Relationship between Escherichia coli strains causing acute cystitis in women and the

973 fecal E. coli population of the host. Journal of Clinical Microbiology. 2008;46(8):2529-34.

974 Epub 2008/05/23. doi: 10.1128/jcm.00813-08. PubMed PMID: 18495863; PubMed

975 Central PMCID: PMCPMC2519474.

976 52. Chen SL, Wu M, Henderson JP, Hooton TM, Hibbing ME, Hultgren SJ, et al.

977 Genomic diversity and fitness of $E$. coli strains recovered from the intestinal and urinary

978 tracts of women with recurrent urinary tract infection. Science Translational Medicine.

979 2013;5(184):184ra60. doi: 10.1126/scitransImed.3005497. PubMed PMID: 23658245;

980 PubMed Central PMCID: PMC3695744.

981 53. Yamamoto S, Tsukamoto T, Terai A, Kurazono H, Takeda Y, Yoshida O. Genetic

982 evidence supporting the fecal-perineal-urethral hypothesis in cystitis caused by

983 Escherichia coli. J Urol. 1997;157(3):1127-9. PubMed PMID: 9072556.

984 54. Russo TA, Stapleton A, Wenderoth S, Hooton TM, Stamm WE. Chromosomal

985 restriction fragment length polymorphism analysis of Escherichia coli strains causing 
986 recurrent urinary tract infections in young women. J Infect Dis. 1995;172(2):440-5. Epub

987 1995/08/01. doi: 10.1093/infdis/172.2.440. PubMed PMID: 7622887.

988 55. Russell CW, Fleming BA, Jost CA, Tran A, Stenquist AT, Wambaugh MA, et al.

989 Context-Dependent Requirements for FimH and Other Canonical Virulence Factors in

990 Gut Colonization by Extraintestinal Pathogenic Escherichia coli. Infection and Immunity.

991 2018;86(3). Epub 2018/01/10. doi: 10.1128/IAI.00746-17. PubMed PMID: 29311232;

992 PubMed Central PMCID: PMCPMC5820936.

993 56. Russell CW, Mulvey MA. The Extraintestinal Pathogenic Escherichia coli Factor

994 Rqll Constrains the Genotoxic Effects of the RecQ-Like Helicase RqlH. PLoS Pathog.

995 2015;11(12):e1005317. doi: 10.1371/journal.ppat.1005317. PubMed PMID: 26636713;

996 PubMed Central PMCID: PMCPMC4670107.

997 57. Russell CW, Richards AC, Chang AS, Mulvey MA. The Rhomboid Protease

998 GlpG Promotes the Persistence of Extraintestinal Pathogenic Escherichia coli within the

999 Gut. Infection and Immunity. 2017;85(6). Epub 2017/04/05. doi: 10.1128/IAI.00866-16.

1000 PubMed PMID: 28373355; PubMed Central PMCID: PMCPMC5442614.

1001 58. Lewis AJ, Richards AC, Mulvey MA. Invasion of Host Cells and Tissues by

1002 Uropathogenic Bacteria. Microbiol Spectr. 2016;4(6). Epub 2017/01/15. doi:

1003 10.1128/microbiolspec.UTI-0026-2016. PubMed PMID: 28087946; PubMed Central

1004 PMCID: PMCPMC5244466.

1005 59. Hagberg L, Hull R, Hull S, McGhee JR, Michalek SM, Svanborg Eden C.

1006 Difference in susceptibility to gram-negative urinary tract infection between $\mathrm{C} 3 \mathrm{H} / \mathrm{HeJ}$

1007 and C3H/HeN mice. Infection and Immunity. 1984;46(3):839-44.

1008 60. Suhs KA, Marthaler BR, Welch RA, Hopkins WJ. Lack of association between

1009 the TIr4 (Lpsd/Lpsd) genotype and increased susceptibility to Escherichia coli bladder

1010 infections in female C3H/HeJ mice. MBio. 2011;2(3):e00094-11. Epub 2011/06/02. doi:

1011 10.1128/mBio.00094-11 e00094-11 [pii] mBio.00094-11 [pii]. PubMed PMID: 21628500;

1012 PubMed Central PMCID: PMC3104495.

1013 61. Hopkins WJ, Elkahwaji J, Kendziorski C, Moser AR, Briggs PM, Suhs KA.

1014 Quantitative trait loci associated with susceptibility to bladder and kidney infections

1015 induced by Escherichia coli in female C3H/HeJ mice. J Infect Dis. 2009;199(3):355-61. 
1016 Epub 2008/12/09. doi: 10.1086/595987. PubMed PMID: 19061424; PubMed Central 1017 PMCID: PMC2683358.

1018 62. Donovan GT, Norton JP, Bower JM, Mulvey MA. Adenylate cyclase and the

1019 cyclic AMP receptor protein modulate stress resistance and virulence capacity of 1020 uropathogenic Escherichia coli. Infection and Immunity. 2013;81(1):249-58. Epub

1021 2012/11/02. doi: 10.1128/iai.00796-12. PubMed PMID: 23115037; PubMed Central 1022 PMCID: PMCPMC3536135.

1023 63. van der Mee-Marquet NL, Blanc DS, Gbaguidi-Haore H, Dos Santos Borges S, 1024 Viboud Q, Bertrand X, et al. Marked increase in incidence for bloodstream infections 1025 due to Escherichia coli, a side effect of previous antibiotic therapy in the elderly. Front 1026 Microbiol. 2015;6:646. doi: 10.3389/fmicb.2015.00646. PubMed PMID: 26175721.

1027 64. Picard B, Garcia JS, Gouriou S, Duriez P, Brahimi N, Bingen E, et al. The link 1028 between phylogeny and virulence in Escherichia coli extraintestinal infection. Infection 1029 and immunity. 1999;67(2):546-53. Epub 1999/01/23. doi: 10.1128/IAI.67.2.5461030 553.1999. PubMed PMID: 9916057; PubMed Central PMCID: PMCPMC96353.

1031 65. Trivedi S, Labuz D, Anderson CP, Araujo CV, Blair A, Middleton EA, et al. 1032 Mucosal-associated invariant T (MAIT) cells mediate protective host responses in 1033 sepsis. Elife. 2020;9. Epub 2020/11/10. doi: 10.7554/eLife.55615. PubMed PMID:

1034 33164745; PubMed Central PMCID: PMCPMC7679140.

1035 66. Mikkola R, Kurland CG. Evidence for demand-regulation of ribosome 1036 accumulation in E. coli. Biochimie. 1991;73(12):1551-6. Epub 1991/12/01. PubMed 1037 PMID: 1805968.

1038 67. Korshunov S, Imlay JA. Detection and quantification of superoxide formed within 1039 the periplasm of Escherichia coli. J Bacteriol. 2006;188(17):6326-34. PubMed PMID: 104016923900.

1041 68. Bower JM, Mulvey MA. Polyamine-mediated resistance of uropathogenic 1042 Escherichia coli to nitrosative stress. J Bacteriol. 2006;188(3):928-33. Epub 2006/01/24. 1043 doi: 188/3/928 [pii] 10.1128/JB.188.3.928-933.2006. PubMed PMID: 16428396.

1044 69. Woolford G, Casselden RJ, Walters CL. Gaseous products of the interaction of 1045 sodium nitrite with procine skeletal muscle. Biochem J. 1972;130(2):82P-3P. Epub 
1046 1972/11/01. doi: 10.1042/bj1300082pb. PubMed PMID: 4664608; PubMed Central 1047 PMCID: PMCPMC1174485.

1048 70. Hassan HM, Fridovich I. Superoxide radical and the oxygen enhancement of the 1049 toxicity of paraquat in Escherichia coli. J Biol Chem. 1978;253(22):8143-8. Epub 1050 1978/11/25. PubMed PMID: 213429.

1051 71. Withman B, Gunasekera TS, Beesetty P, Agans R, Paliy O. Transcriptional 1052 responses of uropathogenic Escherichia coli to increased environmental osmolality 1053 caused by salt or urea. Infection and immunity. 2013;81(1):80-9. Epub 2012/10/24. doi: 1054 10.1128/iai.01049-12. PubMed PMID: 23090957; PubMed Central PMCID:

1055 PMCPMC3536127.

1056 72. Schwan WR. Survival of uropathogenic Escherichia coli in the murine urinary 1057 tract is dependent on OmpR. Microbiology. 2009;155(Pt 6):1832-9. Epub 2009/04/23. 1058 doi: mic.0.026187-0 [pii]

1059 10.1099/mic.0.026187-0. PubMed PMID: 19383700.

1060 73. Urbonavicius J, Qian Q, Durand JM, Hagervall TG, Bjork GR. Improvement of 1061 reading frame maintenance is a common function for several tRNA modifications.

1062 EMBO J. 2001;20(17):4863-73. Epub 2001/09/05. doi: 10.1093/emboj/20.17.4863.

1063 PubMed PMID: 11532950; PubMed Central PMCID: PMC125605.

1064 74. Urbonavicius J, Stahl G, Durand JM, Ben Salem SN, Qian Q, Farabaugh PJ, et

1065 al. Transfer RNA modifications that alter +1 frameshifting in general fail to affect -1

1066 frameshifting. RNA (New York, NY). 2003;9(6):760-8. Epub 2003/05/21. PubMed PMID:

1067 12756333; PubMed Central PMCID: PMC1370442.

1068 75. Howard MT, Shirts BH, Zhou J, Carlson CL, Matsufuji S, Gesteland RF, et al. 1069 Cell culture analysis of the regulatory frameshift event required for the expression of 1070 mammalian antizymes. Genes to Cells. 2001;6(11):931-41. Epub 2001/12/26. PubMed 1071 PMID: 11733031.

1072 76. Grentzmann G, Ingram JA, Kelly PJ, Gesteland RF, Atkins JF. A dual-luciferase 1073 reporter system for studying recoding signals. RNA (New York, NY). 1998;4(4):479-86.

1074 Epub 1998/06/18. PubMed PMID: 9630253; PubMed Central PMCID:

1075 PMCPMC1369633. 
1076 77. Floyd KA, Moore JL, Eberly AR, Good JA, Shaffer CL, Zaver H, et al. Adhesive

1077 fiber stratification in uropathogenic Escherichia coli biofilms unveils oxygen-mediated

1078 control of type 1 pili. PLoS Pathog. 2015;11(3):e1004697. Epub 2015/03/05. doi:

1079 10.1371/journal.ppat.1004697. PubMed PMID: 25738819; PubMed Central PMCID:

1080 PMCPMC4349694.

1081 78. Beebout CJ, Eberly AR, Werby SH, Reasoner SA, Brannon JR, De S, et al.

1082 Respiratory Heterogeneity Shapes Biofilm Formation and Host Colonization in

1083 Uropathogenic Escherichia coli. MBio. 2019;10(2). Epub 2019/04/04. doi:

1084 10.1128/mBio.02400-18. PubMed PMID: 30940709; PubMed Central PMCID:

1085 PMCPMC6445943.

1086 79. Wolf J, Gerber AP, Keller W. tadA, an essential tRNA-specific adenosine

1087 deaminase from Escherichia coli. EMBO J. 2002;21(14):3841-51. Epub 2002/07/12. doi:

1088 10.1093/emboj/cdf362. PubMed PMID: 12110595; PubMed Central PMCID:

1089 PMCPMC126108.

1090 80. Bar-Yaacov D, Mordret E, Towers R, Biniashvili T, Soyris C, Schwartz S, et al.

1091 RNA editing in bacteria recodes multiple proteins and regulates an evolutionarily

1092 conserved toxin-antitoxin system. Genome Res. 2017;27(10):1696-703. Epub

1093 2017/09/03. doi: 10.1101/gr.222760.117. PubMed PMID: 28864459; PubMed Central

1094 PMCID: PMCPMC5630033.

1095 81. Kulesus RR, Diaz-Perez K, Slechta ES, Eto DS, Mulvey MA. Impact of the RNA 1096 chaperone Hfq on the fitness and virulence potential of uropathogenic Escherichia coli.

1097 Infection and Immunity. 2008;76(7):3019-26. Epub 2008/05/07. doi: IAI.00022-08 [pii]

1098 10.1128/IAI.00022-08. PubMed PMID: 18458066.

1099 82. Endres L, Dedon PC, Begley TJ. Codon-biased translation can be regulated by 1100 wobble-base tRNA modification systems during cellular stress responses. RNA biology.

1101 2015;12(6):603-14. Epub 2015/04/22. doi: 10.1080/15476286.2015.1031947. PubMed

1102 PMID: 25892531; PubMed Central PMCID: PMCPMC4615639.

1103 83. Koh CS, Sarin LP. Transfer RNA modification and infection - Implications for

1104 pathogenicity and host responses. Biochim Biophys Acta Gene Regul Mech.

1105 2018;1861(4):419-32. Epub 2018/01/30. doi: 10.1016/j.bbagrm.2018.01.015. PubMed

1106 PMID: 29378328. 
1107 84. Durand JM, Bjork GR, Kuwae A, Yoshikawa M, Sasakawa C. The modified

1108 nucleoside 2-methylthio-N6-isopentenyladenosine in tRNA of Shigella flexneri is

1109 required for expression of virulence genes. J Bacteriol. 1997;179(18):5777-82. Epub

1110 1997/09/19. doi: 10.1128/jb.179.18.5777-5782.1997. PubMed PMID: 9294434; PubMed

1111 Central PMCID: PMCPMC179466.

1112 85. Tsui HC, Winkler ME. Transcriptional patterns of the mutL-miaA superoperon of

1113 Escherichia coli K-12 suggest a model for posttranscriptional regulation. Biochimie.

1114 1994;76(12):1168-77. Epub 1994/01/01. doi: 10.1016/0300-9084(94)90046-9. PubMed

1115 PMID: 7748952.

1116 86. Connolly DM, Winkler ME. Structure of Escherichia coli K-12 miaA and 1117 characterization of the mutator phenotype caused by miaA insertion mutations. J

1118 Bacteriol. 1991;173(5):1711-21. Epub 1991/03/01. doi: 10.1128/jb.173.5.1711-

1119 1721.1991. PubMed PMID: 1999389; PubMed Central PMCID: PMCPMC207322.

1120 87. Dedon PC, Begley TJ. A system of RNA modifications and biased codon use

1121 controls cellular stress response at the level of translation. Chemical Research in

1122 Toxicology. 2014;27(3):330-7. Epub 2014/01/16. doi: 10.1021/tx400438d. PubMed

1123 PMID: 24422464; PubMed Central PMCID: PMCPMC3997223.

1124 88. Chan CT, Pang YL, Deng W, Babu IR, Dyavaiah M, Begley TJ, et al.

1125 Reprogramming of tRNA modifications controls the oxidative stress response by codon-

1126 biased translation of proteins. Nature Comm. 2012;3:937. Epub 2012/07/05. doi:

1127 10.1038/ncomms1938. PubMed PMID: 22760636; PubMed Central PMCID:

1128 PMCPMC3535174.

1129 89. Rehl JM, Shippy DC, Eakley NM, Brevik MD, Sand JM, Cook ME, et al. GidA 1130 expression in Salmonella is modulated under certain environmental conditions. Curr

1131 Microbiol. 2013;67(3):279-85. Epub 2013/04/13. doi: 10.1007/s00284-013-0361-2.

1132 PubMed PMID: 23579313.

1133 90. Pollo-Oliveira L, de Crecy-Lagard V. Can Protein Expression Be Regulated by

1134 Modulation of tRNA Modification Profiles? Biochemistry. 2019;58(5):355-62. Epub

1135 2018/12/05. doi: 10.1021/acs.biochem.8b01035. PubMed PMID: 30511849; PubMed

1136 Central PMCID: PMCPMC6363828. 
1137 91. Wong GT, Bonocora RP, Schep AN, Beeler SM, Lee Fong AJ, Shull LM, et al.

1138 Genome-Wide Transcriptional Response to Varying RpoS Levels in Escherichia coli K-

1139 12. J Bacteriol. 2017;199(7). Epub 2017/01/25. doi: 10.1128/JB.00755-16. PubMed

1140 PMID: 28115545; PubMed Central PMCID: PMCPMC5350281.

1141 92. Battesti A, Majdalani N, Gottesman S. The RpoS-mediated general stress

1142 response in Escherichia coli. Annu Rev Microbiol. 2011;65:189-213. Epub 2011/06/07.

1143 doi: 10.1146/annurev-micro-090110-102946. PubMed PMID: 21639793.

1144 93. Chao Y, Papenfort K, Reinhardt R, Sharma CM, Vogel J. An atlas of Hfq-bound

1145 transcripts reveals 3' UTRs as a genomic reservoir of regulatory small RNAs. EMBO J.

1146 2012;31(20):4005-19. Epub 2012/08/28. doi: 10.1038/emboj.2012.229. PubMed PMID:

$114722922465 ;$ PubMed Central PMCID: PMCPMC3474919.

1148 94. Pan T. Adaptive translation as a mechanism of stress response and adaptation.

1149 Annual Review of Genetics. 2013;47:121-37. Epub 2013/08/31. doi: 10.1146/annurev-

1150 genet-111212-133522. PubMed PMID: 23988117; PubMed Central PMCID:

1151 PMCPMC4109725.

1152 95. Drummond DA, Wilke CO. The evolutionary consequences of erroneous protein

1153 synthesis. Nat Rev Genet. 2009;10(10):715-24. doi: 10.1038/nrg2662. PubMed PMID:

1154 19763154; PubMed Central PMCID: PMCPMC2764353.

1155 96. Moura GR, Carreto LC, Santos MA. Genetic code ambiguity: an unexpected

1156 source of proteome innovation and phenotypic diversity. Curr Opin Microbiol.

1157 2009;12(6):631-7. doi: 10.1016/j.mib.2009.09.004. PubMed PMID: 19853500.

1158 97. Mohler K, Ibba M. Translational fidelity and mistranslation in the cellular response

1159 to stress. Nature Microbiology. 2017;2:17117. Epub 2017/08/25. doi:

1160 10.1038/nmicrobiol.2017.117. PubMed PMID: 28836574; PubMed Central PMCID:

1161 PMCPMC5697424.

1162 98. Blango MG, Mulvey MA. Persistence of uropathogenic Escherichia coli in the

1163 face of multiple antibiotics. Antimicrob Agents Chemother. 2010;54(5):1855-63. Epub

1164 2010/03/17. doi: AAC.00014-10 [pii] 10.1128/AAC.00014-10. PubMed PMID: 20231390;

1165 PubMed Central PMCID: PMC2863638.

1166 99. Mulvey MA, Lopez-Boado YS, Wilson CL, Roth R, Parks WC, Heuser J, et al.

1167 Induction and evasion of host defenses by type 1-piliated uropathogenic Escherichia 
1168 coli. Science (New York, NY). 1998;282(5393):1494-7. Epub 1998/11/20. PubMed

1169 PMID: 9822381.

1170 100. Blango MG, Ott EM, Erman A, Veranic P, Mulvey MA. Forced resurgence and

1171 targeting of intracellular uropathogenic Escherichia coli reservoirs. PloS One.

1172 2014;9(3):e93327. Epub 2014/03/29. doi: 10.1371/journal.pone.0093327. PubMed

1173 PMID: 24667805; PubMed Central PMCID: PMCPMC3965547.

1174 101. Parkinson JS. cheA, cheB, and cheC genes of Escherichia coli and their role in

1175 chemotaxis. J Bacteriol. 1976;126(2):758-70. Epub 1976/05/01. doi:

1176 10.1128/JB.126.2.758-770.1976. PubMed PMID: 770453; PubMed Central PMCID:

1177 PMCPMC233211.

1178 102. Eto DS, Gordon HB, Dhakal BK, Jones TA, Mulvey MA. Clathrin, AP-2, and the

1179 NPXY-binding subset of alternate endocytic adaptors facilitate FimH-mediated bacterial

1180 invasion of host cells. Cellular Microbiology. 2008;10(12):2553-67. Epub 2008/08/30.

1181 doi: 10.1111/j.1462-5822.2008.01229.x. PubMed PMID: 18754852.

1182 103. Miles ZD, Myers WK, Kincannon WM, Britt RD, Bandarian V. Biochemical and

1183 Spectroscopic Studies of Epoxyqueuosine Reductase: A Novel Iron-Sulfur Cluster- and

1184 Cobalamin-Containing Protein Involved in the Biosynthesis of Queuosine. Biochemistry.

1185 2015;54(31):4927-35. Epub 2015/08/01. doi: 10.1021/acs.biochem.5b00335. PubMed

1186 PMID: 26230193; PubMed Central PMCID: PMCPMC4753064.

1187 104. Miles ZD, McCarty RM, Molnar G, Bandarian V. Discovery of epoxyqueuosine

1188 (oQ) reductase reveals parallels between halorespiration and tRNA modification. Proc

1189 Natl Acad Sci U S A. 2011;108(18):7368-72. Epub 2011/04/20. doi:

1190 10.1073/pnas.1018636108. PubMed PMID: 21502530; PubMed Central PMCID:

1191 PMCPMC3088584.

1192 105. Keseler IM, Mackie A, Santos-Zavaleta A, Billington R, Bonavides-Martinez C,

1193 Caspi R, et al. The EcoCyc database: reflecting new knowledge about Escherichia coli

1194 K-12. Nucleic acids research. 2017;45(D1):D543-D50. Epub 2016/12/03. doi:

1195 10.1093/nar/gkw1003. PubMed PMID: 27899573; PubMed Central PMCID:

1196 PMCPMC5210515.

1197 106. Szklarczyk D, Gable AL, Lyon D, Junge A, Wyder S, Huerta-Cepas J, et al.

1198 STRING v11: protein-protein association networks with increased coverage, supporting 
1199 functional discovery in genome-wide experimental datasets. Nucleic Acids Research.

1200 2019;47(D1):D607-D13. Epub 2018/11/27. doi: 10.1093/nar/gky1131. PubMed PMID:

1201 30476243; PubMed Central PMCID: PMCPMC6323986.

1202 107. Kelley LA, Mezulis S, Yates CM, Wass MN, Sternberg MJ. The Phyre2 web

1203 portal for protein modeling, prediction and analysis. Nat Protoc. 2015;10(6):845-58.

1204 Epub 2015/05/08. doi: 10.1038/nprot.2015.053. PubMed PMID: 25950237; PubMed

1205 Central PMCID: PMCPMC5298202.

1206 108. Mulvey MA, Schilling JD, Hultgren SJ. Establishment of a persistent Escherichia

1207 coli reservoir during the acute phase of a bladder infection. Infection and Immunity.

1208 2001;69(7):4572-9. PubMed PMID: 11402001.

1209 109. Guzman LM, Belin D, Carson MJ, Beckwith J. Tight regulation, modulation, and

1210 high-level expression by vectors containing the arabinose PBAD promoter. J Bacteriol.

1211 1995;177(14):4121-30. Epub 1995/07/01. doi: 10.1128/jb.177.14.4121-4130.1995.

1212 PubMed PMID: 7608087; PubMed Central PMCID: PMCPMC177145.

1213 110. Zhou Q, Ames P, Parkinson JS. Mutational analyses of HAMP helices suggest a

1214 dynamic bundle model of input-output signalling in chemoreceptors. Mol Microbiol.

1215 2009;73(5):801-14. Epub 2009/08/07. doi: 10.1111/j.1365-2958.2009.06819.x. PubMed

1216 PMID: 19656294; PubMed Central PMCID: PMCPMC2749569.

1217 


\section{FIGURE LEGENDS}

1219 Figure 1. MiaA promotes ExPEC fitness and virulence within diverse host niches.

1220 (A) MiaA and MiaB act sequentially to modify tRNA molecules that recognize UNN 1221 codons; modified from [37]. DMAPP, dimethylallyl diphosphate; SAM, S-

1222 adenosylmethionine; SAH, S-adenosylhomocysteine; Cys, cysteine.

1223 (B and C) To assess gut colonization, adult BALB/c mice were inoculated via oral gavage 1224 with $\sim 10^{9}$ CFU of a 1:1 mixture of (B) UTI89 and UTI89 $\triangle$ miaA or (C) UTI89 and $1225 \mathrm{UTI} 89 \Delta m i a B$. Fecal titers were determined at the indicated time points and used to 1226 calculate competitive indices $(\mathrm{Cl}) .{ }^{* *}, P<0.001{ }^{* * * *}, P<0.0001$ by one sample $t$-tests. $1227 n=7-8$ mice from two independent experiments.

1228 (D) The bladders of adult female $\mathrm{CBA} / \mathrm{J}$ mice were inoculated via transurethral 1229 catheterization with $\sim 10^{7}$ CFU of UTI89, UTI89 $\Delta$ miaA, or UTI89 $\Delta$ miaB. Mice were 1230 sacrificed 3 days later and bacterial titers within the bladders were determined by plating 1231 tissue homogenates. ${ }^{* *}, P<0.01$ by Mann Whitney $U$ tests; $n \geq 19$ mice per group from 1232 at least three independent experiments. In B, C, and D, bars indicate median values; dots 1233 represent individual mice.

1234 (E) Kaplan Meier survival curves of C57BI/6 mice inoculated via i.p. injections with $\sim 10^{7}$ 1235 CFU of UTI89 (black line), UTI89 $\Delta$ miaA (blue), or UTI89 $\Delta$ miaB (orange). ${ }^{* * * *}, P<0.0001$ 1236 by Log-rank Mantel Cox test for UTI89 versus UTI89 $\Delta$ miaA; $n=13$ mice per group from 1237 two independent experiments. 


\section{Figure 1}

A

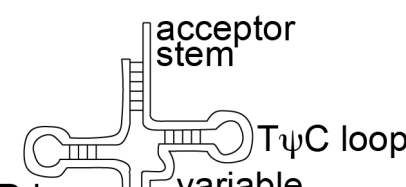

$D$ loop variable

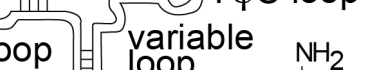

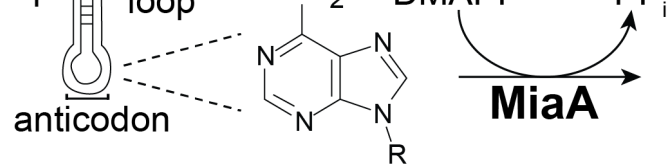<smiles>[R]n1cnc2c(NCC=C(C)C)ncnc21</smiles>

\section{tRNA Adenosine-37}

${ }^{i} \mathrm{~A}-37$
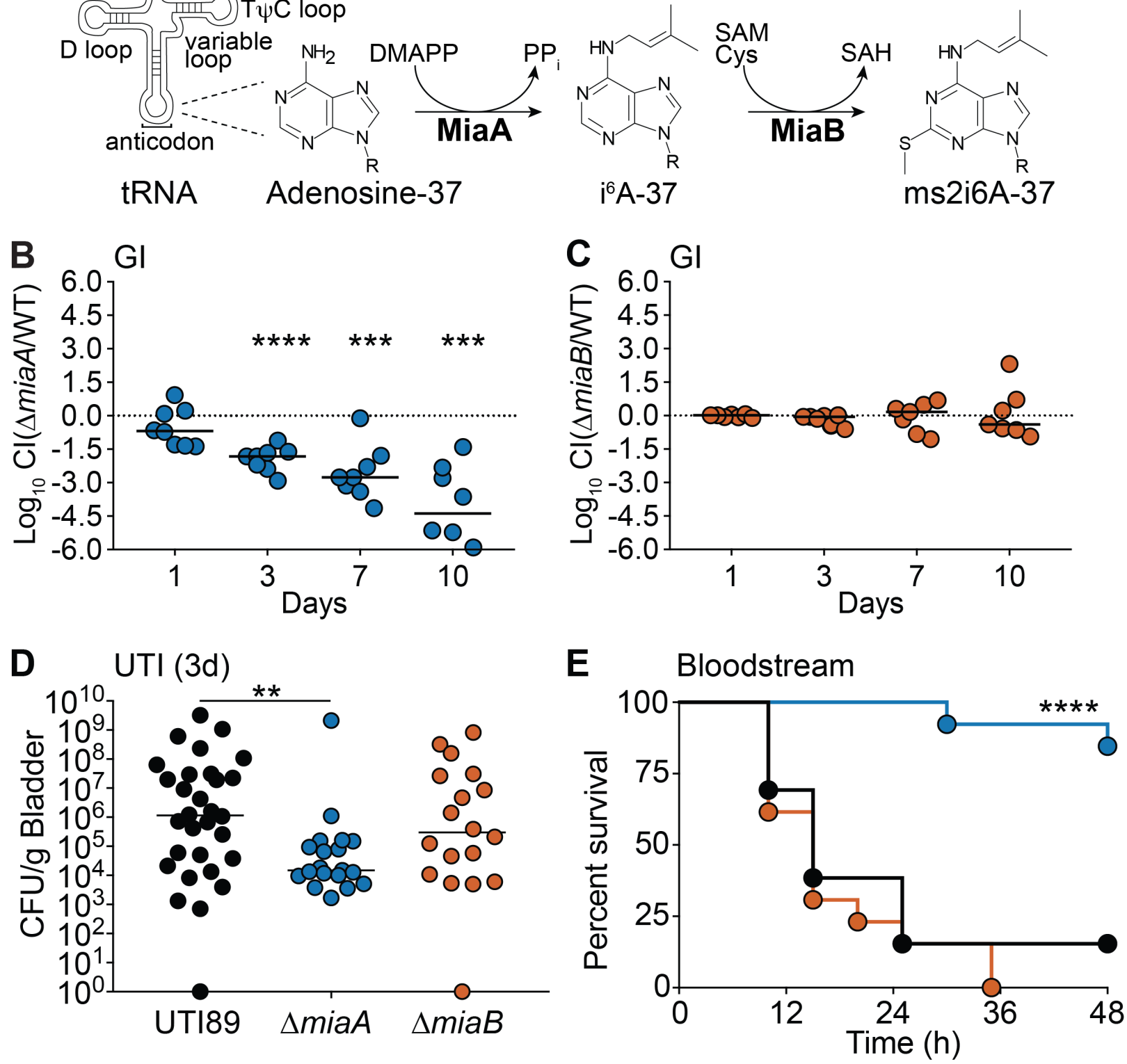
1238 Figure 2. Deletion of miaA limits growth of UTI89 in rich medium and lowers 1239 resistance to oxidative and nitrosative stress.

1240 (A-F) Graphs indicate mean growth of UTI89, UTI89 $\Delta m i a A$, and UTI89 $\Delta m i a B$ in shaking 1241 cultures with modified M9 media, LB, MES-LB, MES-LB with 1 or $2 \mathrm{mM}$ ASN, or LB with $12421 \mathrm{mM} \mathrm{MV}$.

1243 (G and H) Curves show mean growth of UTI89 and UTI89 $\triangle$ miaA carrying pMiaA $A_{\text {nat }}$ (with 1244 miaA expressed from its native promoter) or the empty vector control pACYC184 in LB 1245 containing (G) $2 \mathrm{mM}$ ASN or $(\mathrm{H}) 1 \mathrm{mM} \mathrm{MV}$.

1246 Each curve shows the means of results from four replicates, and are representative of 1247 three independent experiments. 
Figure 2

A
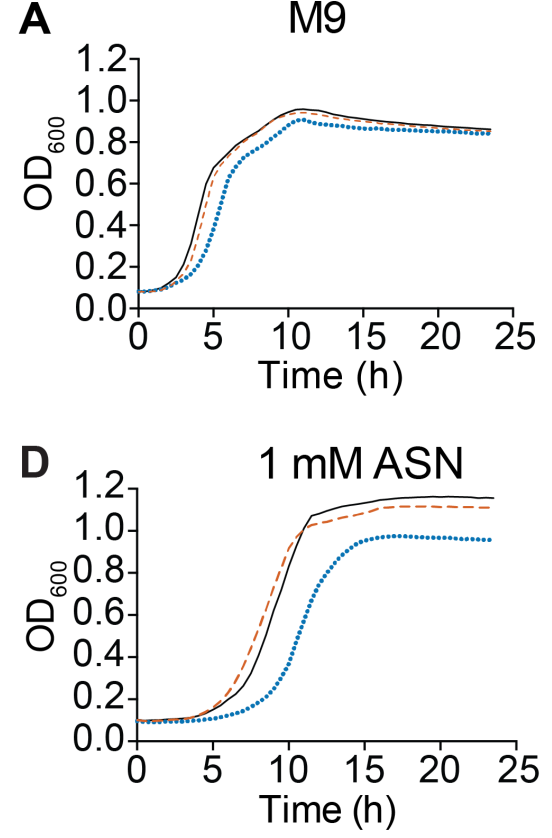

B
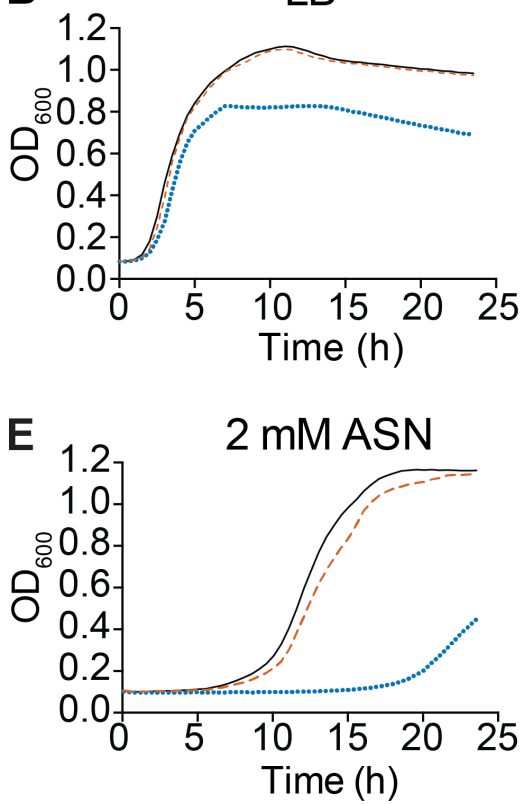

C MES-LB (pH 5.0)
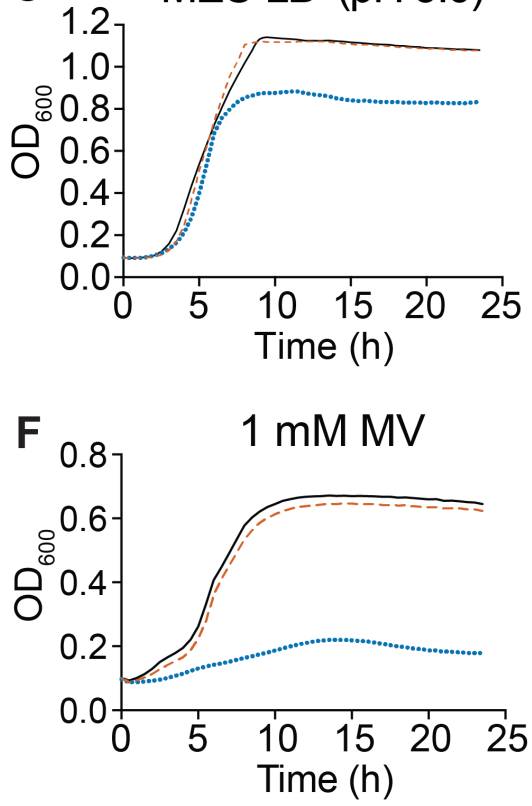

— UTI89 -....... $\Delta$ miaA -- $\Delta$ miaB

—UTI89/pACYC184

- UTI89/pMiaA

....... $\triangle$ miaA/pACYCY 184

-.. $\Delta$ miaA/pMiaA ${ }_{\text {nat }}$
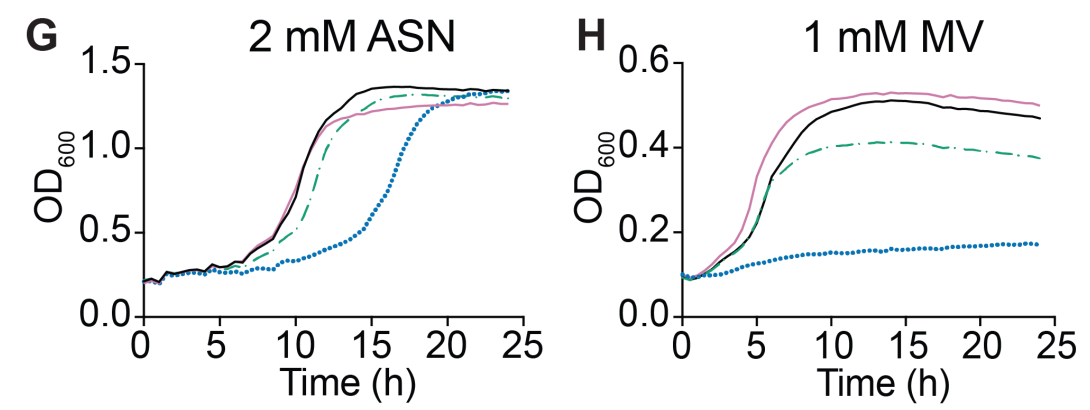
1248 Figure 3. MiaA enhances the resistance of UTI89 to osmotic stress.

1249 (A) Bacteria were grown to stationary phase in LB, pelleted, and resuspended in $\mathrm{ddd}_{2} \mathrm{O}$.

1250 The mean numbers $( \pm$ SEM) of surviving bacteria recovered at the indicated time points

1251 are graphed as the percentage of the bacteria present immediately after resuspension in

$1252 \mathrm{ddH}_{2} \mathrm{O}$ (time 0 ). $P$-values were determined by two-way ANOVA with the Geisser-

1253 Greenhouse correction; $\mathrm{n}=3$ independent assays each done in triplicate.

1254 (B) Bars indicate mean numbers of bacteria $( \pm S D)$ that survived 2 hours in $\mathrm{ddH}_{2} \mathrm{O}$ with

$12550.1 \%$ glucose, calculated as a percent of the inoculum. $P$ values were determined, relative

1256 to the control strain UTI89/pMiaAnat, by unpaired $t$-tests with Welch's correction; $\mathrm{n}=3$

1257 independent assays.

1258 (C and D) Curves show growth of the indicated strains in $\mathrm{LB}$ plus $5 \% \mathrm{NaCl}$, as measured 1259 by $\mathrm{OD}_{600}$. Data are representative of at least three independent experiments performed 1260 in quadruplicate.
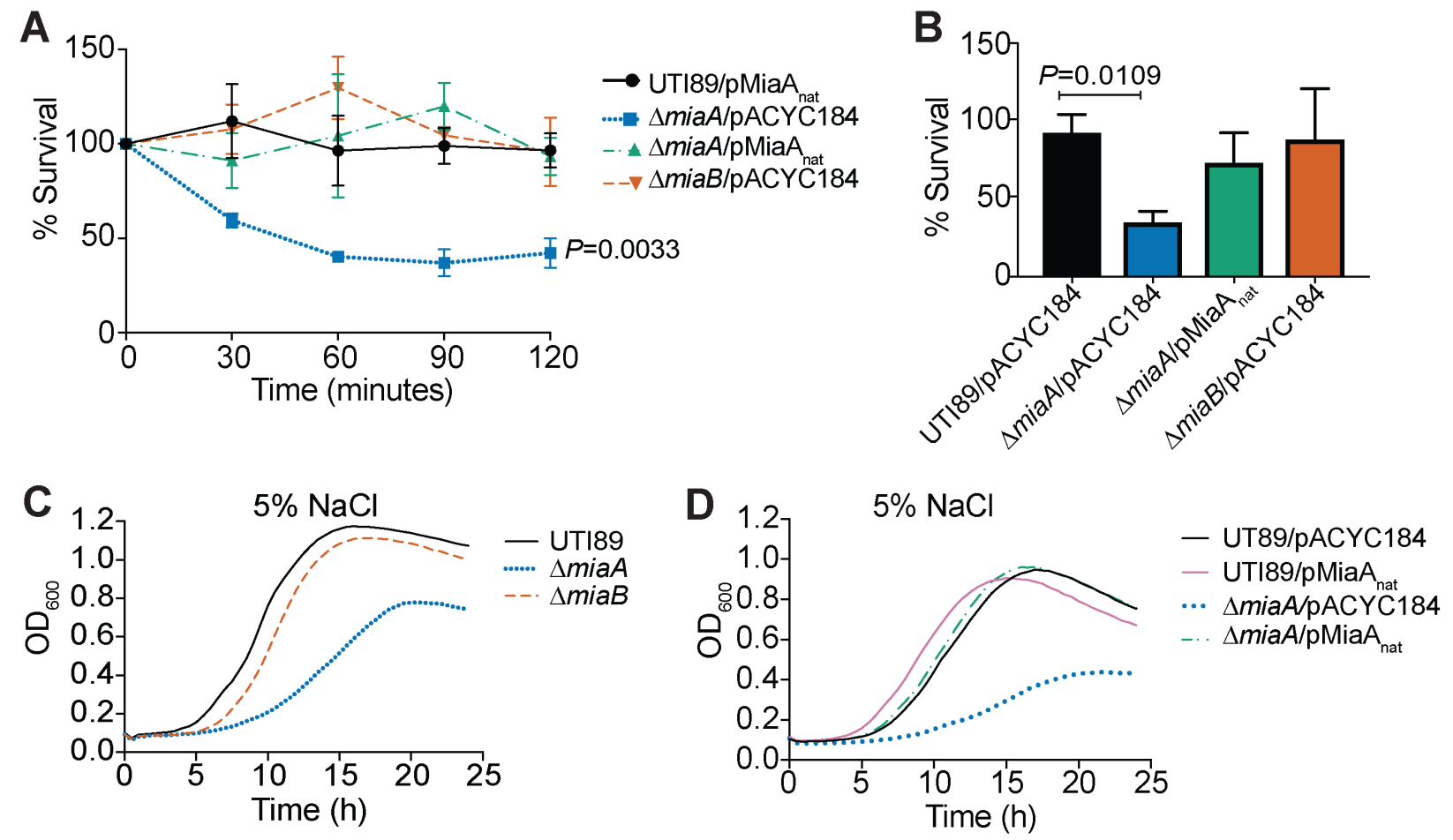
1262 Figure 4. High salt stress downregulates MiaA translation and reduces $\mathrm{ms}^{2} \mathrm{i}^{6} \mathrm{~A}$

1263 levels.

1264 (A) Top panel shows schematic of the experimental setup. UTI89/pMiaA-Flagnat was 1265 diluted from overnight cultures into fresh LB and grown shaking for 2.5 hours at $37^{\circ} \mathrm{C}$ prior

1266 to resuspension in LB or LB $+5 \% \mathrm{NaCl}$. Incubations were continued for the indicated

1267 times before samples were collected and analyzed by western blots using anti-Flag and 1268 anti-E. coli (loading control) antibodies (bottom panel).

1269 (B) Top panel summarizes the experimental setup. Overnight cultures of UTI89/pMiaA-

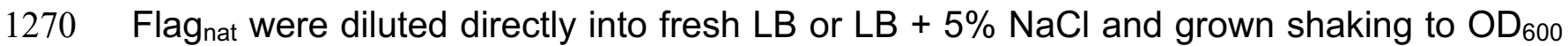

1271 of 0.5 prior to processing for western blot analysis (bottom panel).

1272 (C and D) UTI89 from mid-logarithmic cultures in LB was resuspended in LB or LB + 5\%

$1273 \mathrm{NaCl}$ and one hour later the evels of $\mathrm{mia} A$ and miaB transcripts were determined by RT1274 qPCR. Bars indicate mean values from 9 independent replicates, each with two technical 1275 replicates. ${ }^{*}, P<0.05 ;{ }^{* * *}, P<0.001$ by Mann-Whitney $U$ tests.

1276 (E and $\mathbf{F}$ ) Graphs show relative levels of $i^{6} \mathrm{~A}$ and $\mathrm{ms}^{2} \mathrm{i}^{6} \mathrm{~A}$ recovered from UTI89 and 1277 UTI89 $\triangle$ miaA following growth to $\mathrm{OD}_{600}$ of 0.5 in LB or LB $+5 \% \mathrm{NaCl}$, as determined by 1278 LC-MS. ${ }^{* *}, P<0.01 ;{ }^{* * *}, P<0.001 ;{ }^{* * * *}, P<0.0001$ by unpaired $t$ tests. Bars indicate 1279 median values from 4 to 6 independent replicates. 


\section{Figure 4}

A

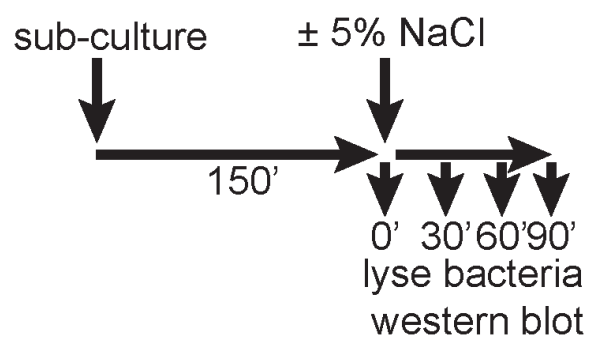

$0 \mathrm{~min} 30 \mathrm{~min} 60 \mathrm{~min} 90 \mathrm{~min}$

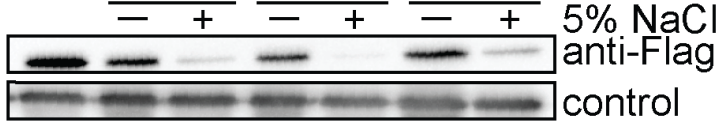

B

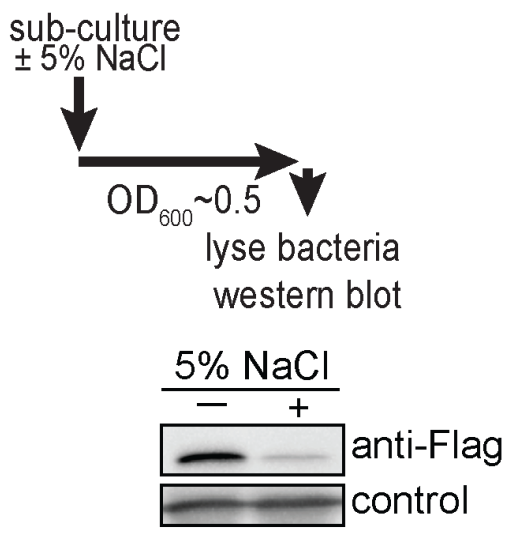

C

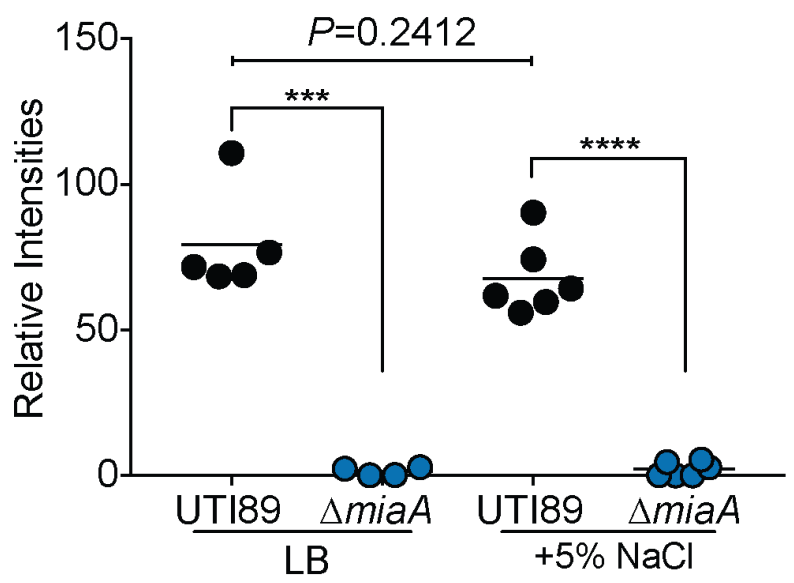

D

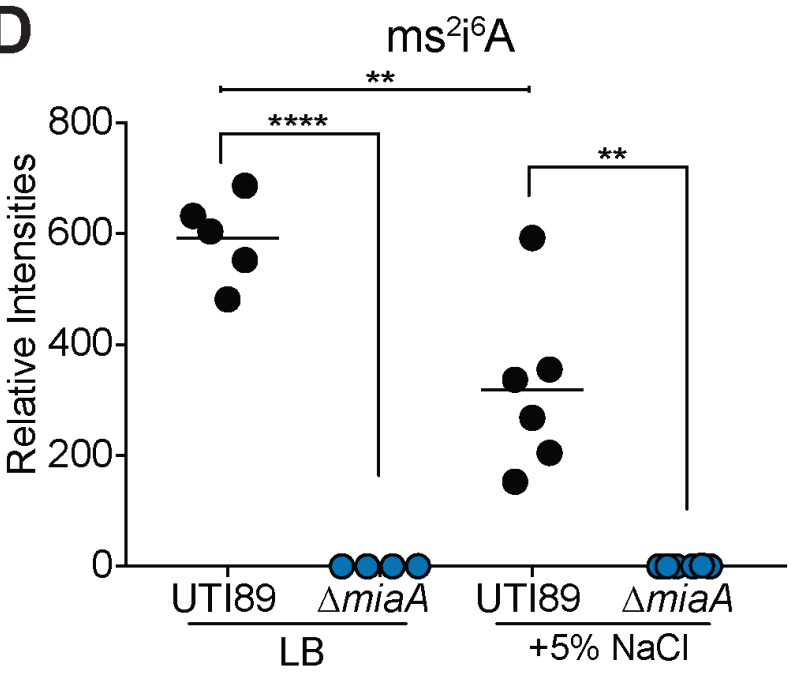


1280 Figure 5. Overexpression of MiaA reduces ExPEC stress resistance.

1281 (A) Relative levels of $\mathrm{i}^{6} \mathrm{~A}$ and $\mathrm{ms}^{2} \mathrm{i}^{6} \mathrm{~A}$ in UTI89 carrying either pMiaAptac or the empty vector

1282 control pRR48 following growth to $\mathrm{OD}_{600}$ of 0.5 in LB with $1 \mathrm{mM}$ IPTG, as quantified by

1283 LC-MS. ${ }^{* * * *}, P<0.0001$ by unpaired $t$ test; $\mathrm{n}=5$ to 6 independent replicates per group.

1284 (B-F) Curves depict growth of UTI89 carrying pMiaAptac or the empty vector control

1285 pRR48 in LB, LB + 5\% NaCl, LB + 1mM MV, MES-LB, or MES-LB + 1 mM ASN. Cultures

1286 were grown shaking at $37^{\circ} \mathrm{C}$ with IPTG added in ten-fold increments from 0 to $1000 \mu \mathrm{M}$,

1287 as indicated. Each curve depicts the means of results from a single experiment and is

1288 representative of at least three independent experiments carried out in quadruplicate.

A

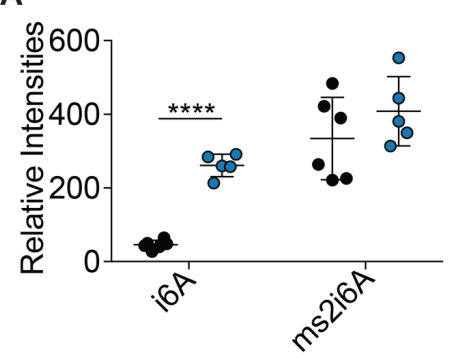

- UTI89/pRR48

- UTI89/pMiaA ${ }_{\text {Ptac }}$
B

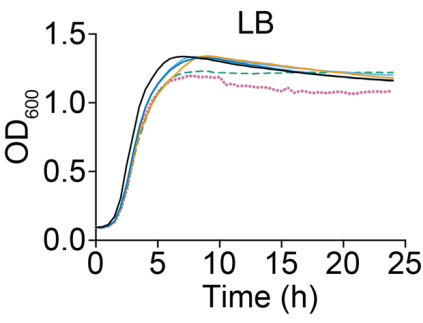

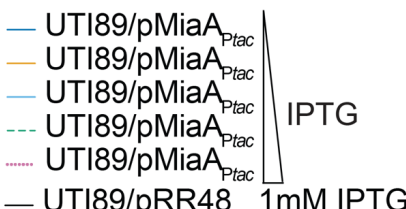

C

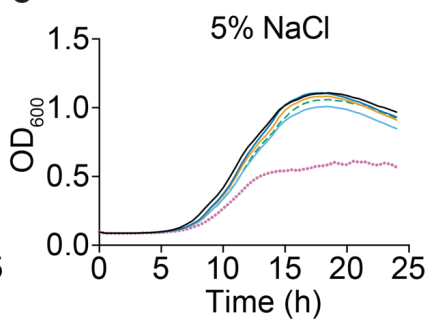

E

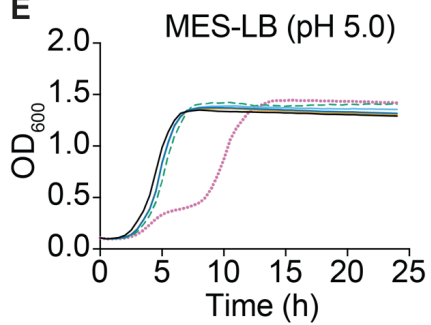

D

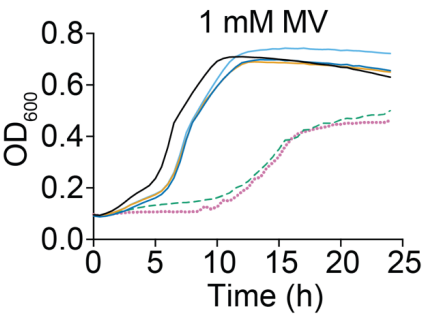

F

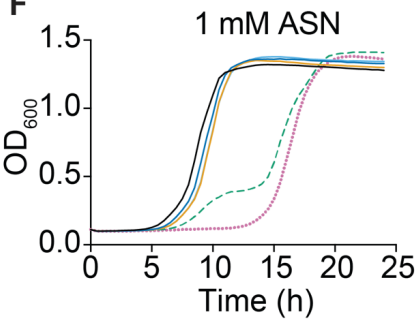


1289 Figure 6. Changing levels of MiaA increase frameshifting.

1290 (A) Diagram depicts the structures of the dual luciferase reporters, with specific intergenic

1291 linker sequences and premature stop codons (underlined red) indicated below. Each

1292 linker contains MiaA-sensitive UNN codons.

1293 (B and C) Graphs show results from +1 and -1 frameshifting assays with UTI89, 1294 UTI89 $\Delta m i a A$, UTI89/pRR48, or UTI89/pMiaAptac carrying one of the dual luciferase 1295 reporter constructs. Bacteria were grown shaking at $37^{\circ} \mathrm{C}$ in LB, with $1 \mathrm{mM} \mathrm{IPTG}$ included 1296 for UTI89/pRR48 and UTI89/pMiaAptac. After reaching an $\mathrm{OD}_{600}$ of $\sim 0.2,0.2 \%$ arabinose 1297 was added to induce expression of the luciferases. At an $\mathrm{OD}_{600}$ of 0.5 , translational error 1298 rates were quantified by determining the ratio of firefly to renilla luciferase activities in 1299 bacteria carrying the +1 (Az1) and -1 (HIV) reporter constructs. Results were normalized 1300 using the ratio of firefly to renilla luciferase activity in bacteria carrying control plasmids in 1301 which the luciferases are in-frame. ${ }^{* *}, P<0.01 ;{ }^{* * * *}, P<0.0001$ by two-tailed unpaired $t$ 1302 tests; $n=10-14$ independent replicates. 
Figure 6

A

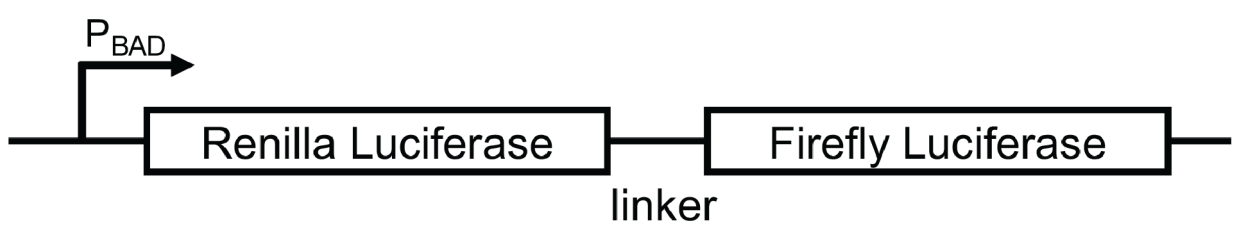

pCWR43 (+1 Frameshift Reporter, Az1-derived linker)

...TCTCCCTCCACTGCTGTAGTAACCCGGGTCCGGGGCCTCGGTGGTGCTCCTGATGCCCCTCA CCCACCCCTGAAGATCCCAGGTGGGCGAGGGAATAGTCAGAGGGATCACAATCTTTCAGCT...

pCWR45 (-1 Frameshift Reporter, HIV gag-pol-derived linker)

... AATTTTTTAGGGAAGATCTGGCCTTCCCACAAGGGGAGGCCAGGGAATTTTCTTCACTGA...
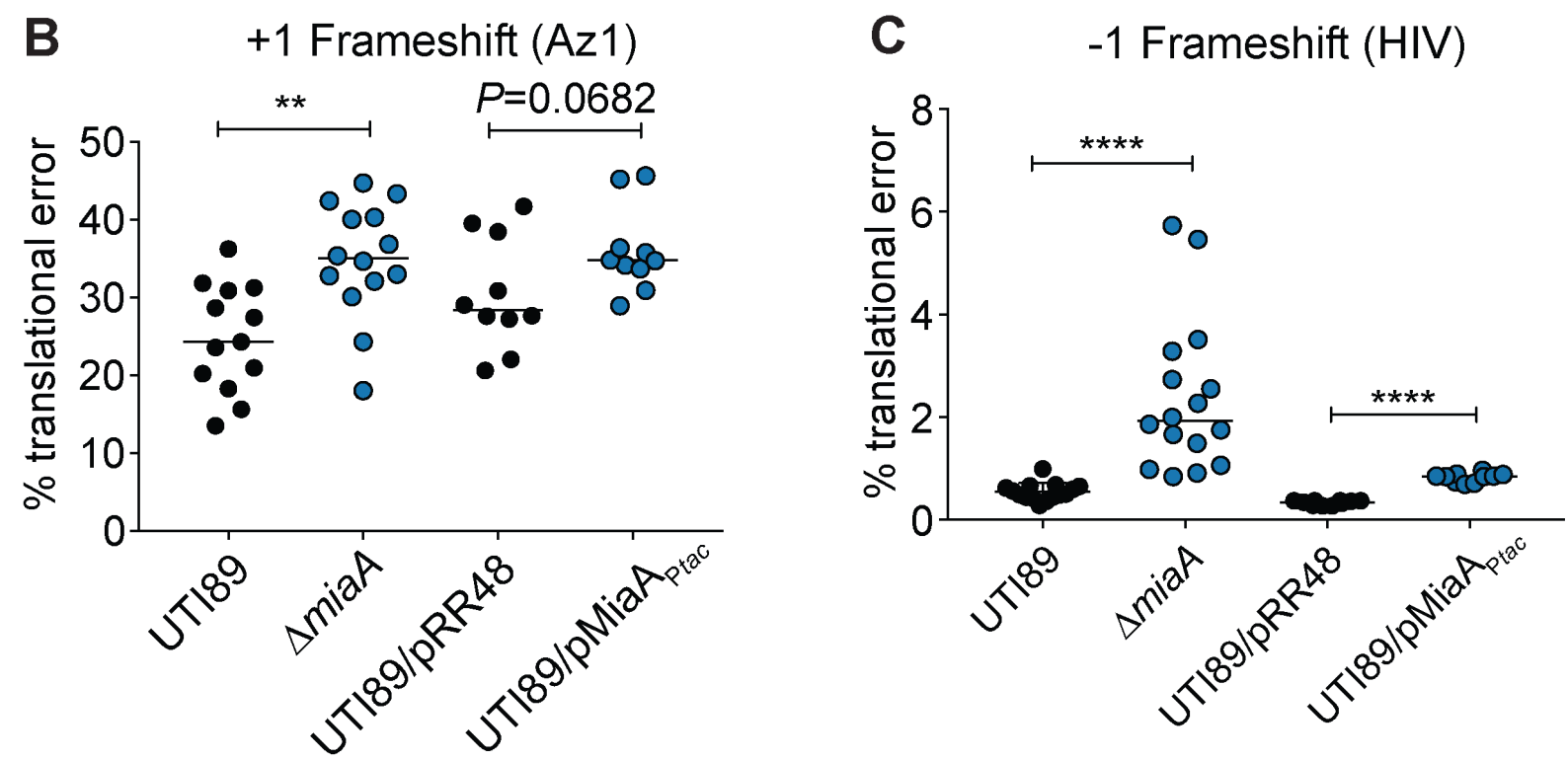
1304 Figure 7. Altering MiaA levels changes the spectrum of expressed proteins.

1305 (A and B) Venn diagrams indicate numbers of unique and shared proteins detected in 1306 wild-type UTI89 versus UTI89 $4 m i a A$, or in UTI89/pRR48 versus UTI89/pMiaAptac,

1307 following growth to $\mathrm{OD}_{600}$ of 0.5 in LB. IPTG $(1 \mathrm{mM})$ was included in the UTI89/pRR48

1308 and UTI89/pMiaAptac cultures. Relative protein levels were determined by MudPIT.

1309 (C and D) Volcano plots show relative protein levels (Log2-fold change) versus P-values

1310 (-Log10). Proteins from UTI89 $\triangle$ miaA were quantified relative to the wild-type strain, while 1311 proteins levels from UTI89/pMiaAptac were assessed relative to UTI89/pRR48. The 1312 vertical dotted lines denote a 2-fold change, while the horizontal dotted lines indicate a $P$ 1313 value of 0.05 . Blue dots indicate proteins that were significantly changed $(P<0.05)$ by at 1314 least 2-fold. $P$ values were determined by Student's $t$ tests; $n=4$ independent replicates 1315 for each group. 
Figure 7
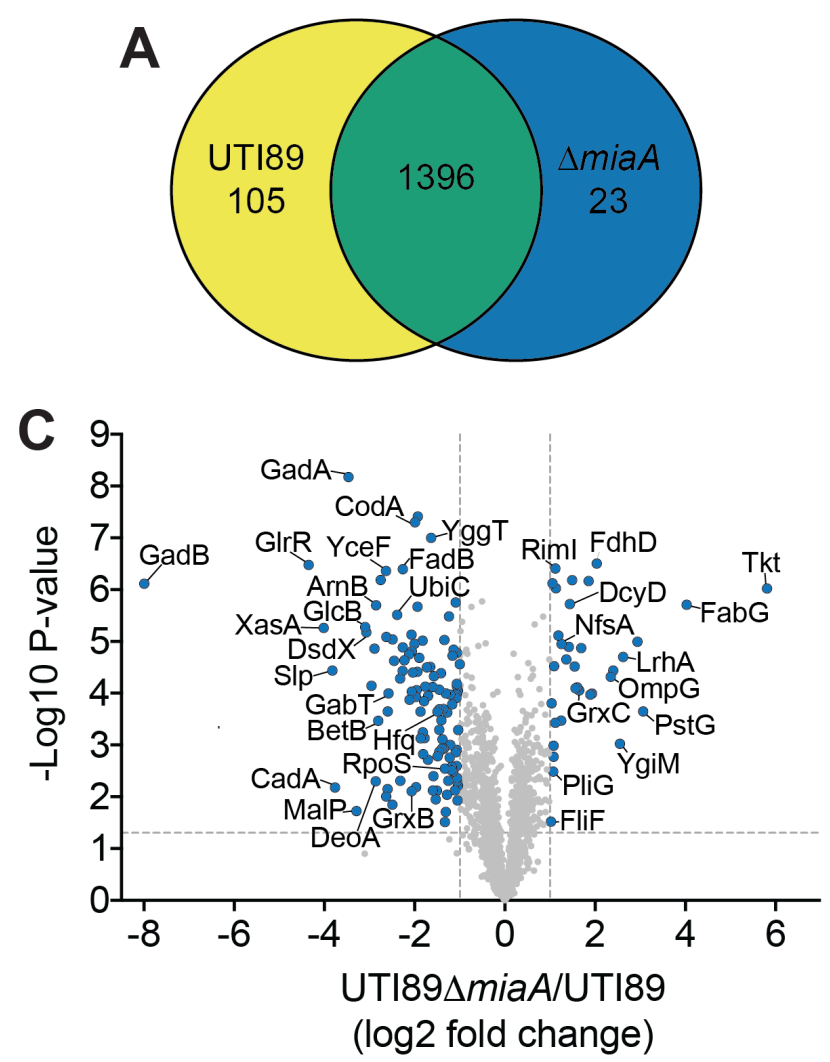

B
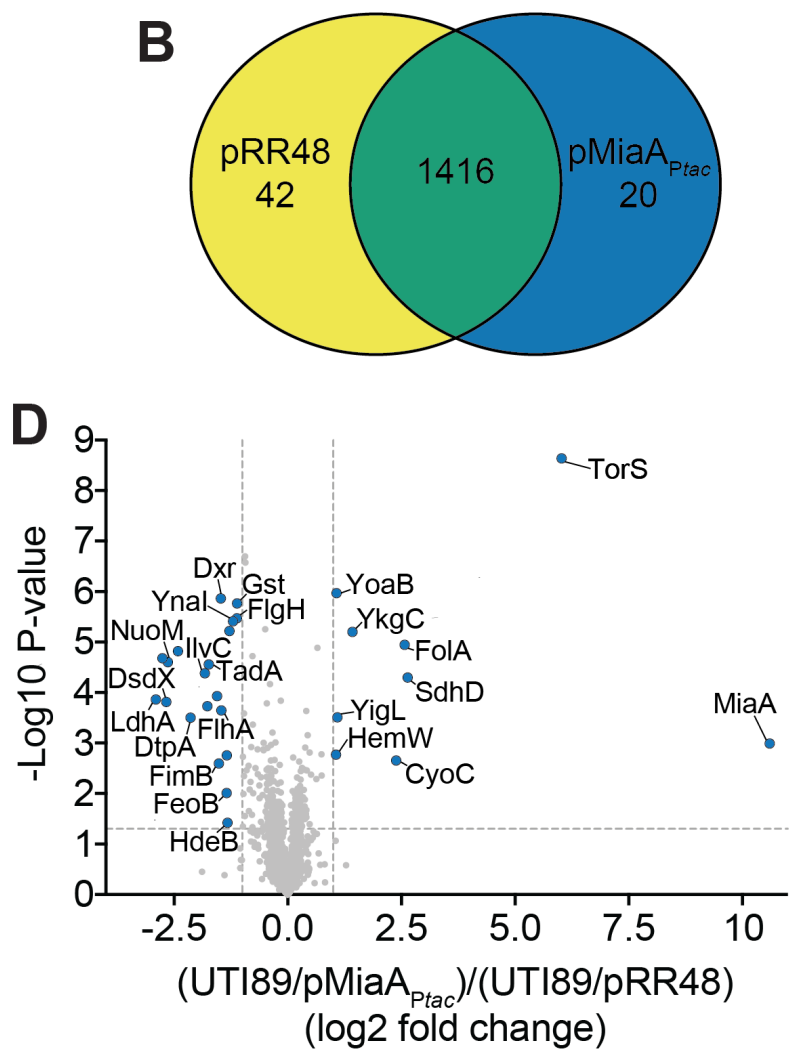
1316 Figure 8. MiaA regulates ExPEC biofilm development.

1317 Images show biofilms formed by wild-type UTI89 and its derivatives after 14 days of

1318 growth at room temperature on YESCA plates. Photos are representative of at least three

1319 independent replicates. Scale bar, $1 \mathrm{~cm}$.

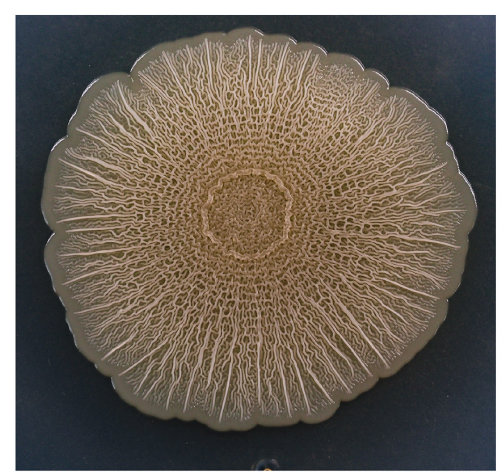

UTI89/pRR48

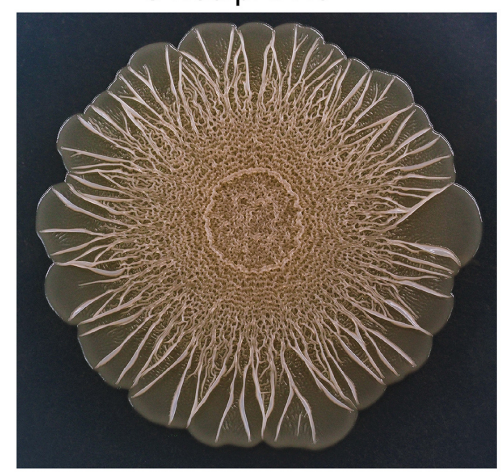

UTI89 $\Delta$ miaA/pRR48

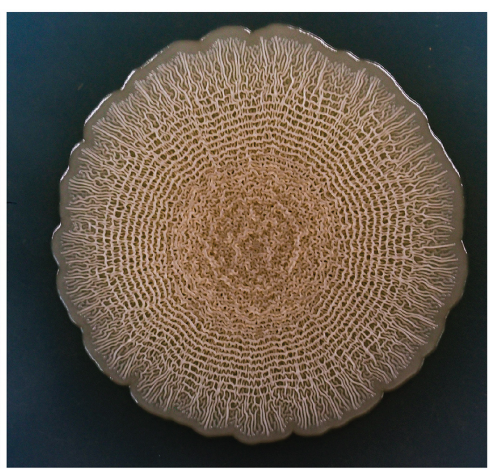

UTI89 $\Delta$ miaB/pRR48

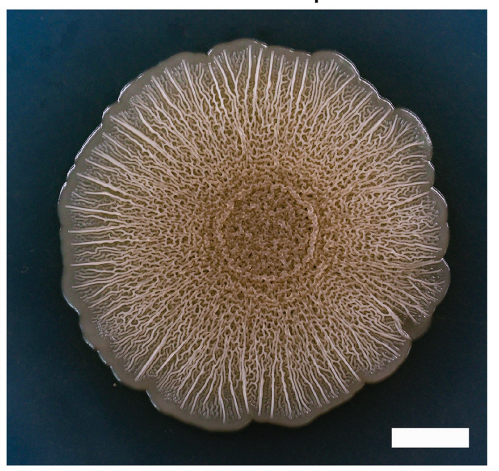

UTI89 $\Delta$ miaA/pMiaA ${ }_{\text {Ptac }}$ 
1320 Figure 9. UNN-Leu codon usage ratios vary among MiaA-sensitive transcripts.

1321 (A and B) Plots show relative protein levels (Log2-fold change) versus UNN-Leu codon

1322 usage ratios (UNN Leu/total Leu codons per open reading frame). Proteins in (A)

1323 UTI89 $\triangle m i a A$ were quantified by MudPIT relative to wild-type UTI89, while proteins levels

1324 in (B) UTI89/pMiaAptac were calculated relative to UTI89/pRR48, as in Fig. 7. Purple dots

1325 denote proteins that were significantly changed ( $P<0.05$, by Student's $t$ tests) by at least

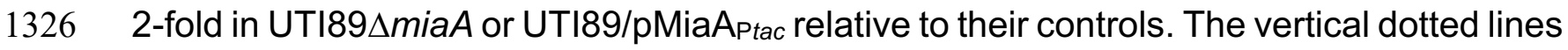

1327 are placed at the 2-fold change cutoffs. The green and black horizontal dashed lines

1328 indicate the average ratios of UNN-Leu codons relative to total Leu codons for all open

1329 reading frames encoded by the K-12 strain MG1655 and UTI89, respectively.
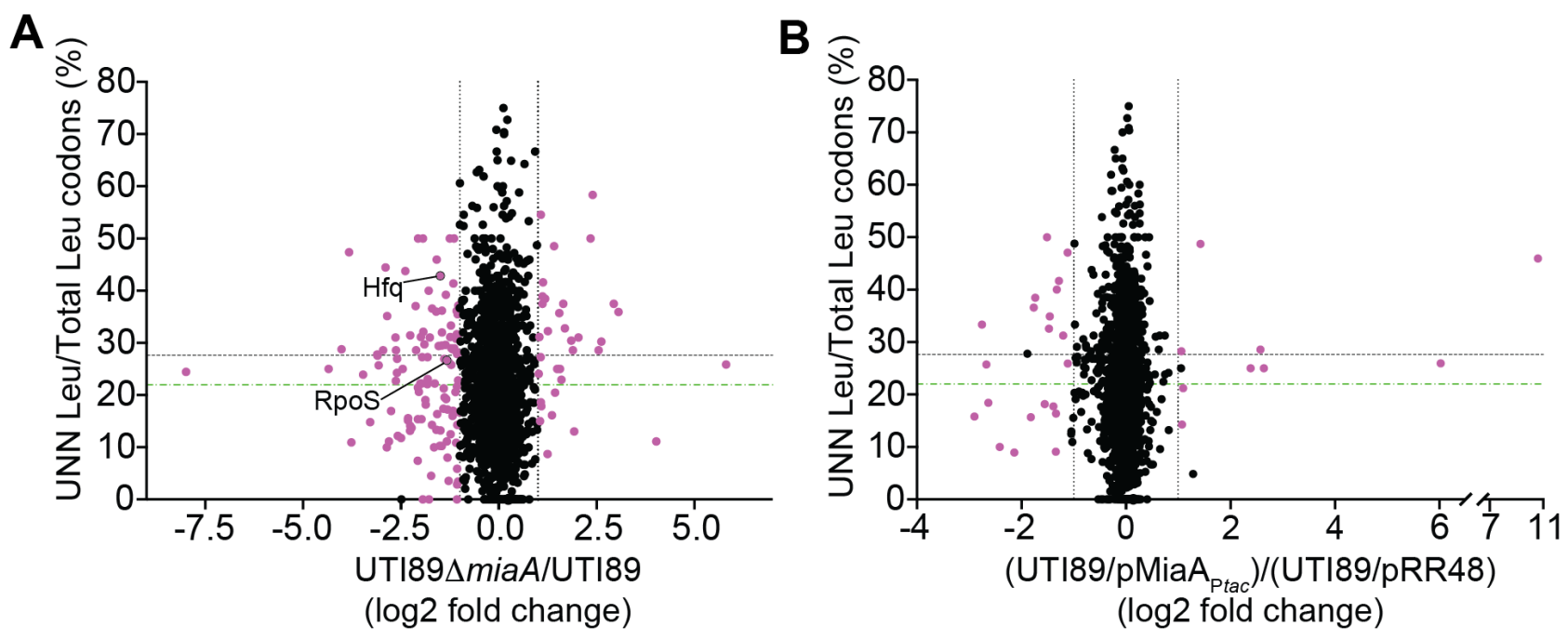
SUPPLEMENTAL INFORMATION

1331 Supplemental Figure S1: MiaA and MiaB do not affect the ability of ExPEC to bind,

1332 invade, or persist intracellularly within bladder epithelial cells.

1333 Human bladder epithelial cells (5637 cells) were infected with UTI89, UTI89 $\Delta$ miaA, or

1334 UTI89 $\triangle$ miaB for $2 \mathrm{~h}$, followed by a second 2-h incubation in the presence of the

1335 bactericidal, host cell-impermeable antibiotic gentamicin $(100 \mu \mathrm{g} / \mathrm{ml})$. Graphs show $(\mathbf{A})$

1336 the levels of host cell-associated bacteria prior to the addition of gentamicin, (B) and the

1337 relative numbers of intracellular bacteria recovered after the 2-h incubation in media

1338 containing gentamicin. (C) Longer-term bacterial persistence within the bladder cells was

1339 assessed by continued incubation of infected host cells for an additional $12 \mathrm{~h}$ with

1340 gentamicin. For the longer persistence assays, a submaximal concentration of gentamicin

$1341(10 \mu \mathrm{g} / \mathrm{ml})$ was used to prevent extracellular growth of UPEC while limiting possible

1342 leaching of the antibiotic into the host cells. Data are expressed relative to wild-type

1343 UTI89, with bars indicating median values from 9 to 18 independent experiments

1344 performed in triplicate.

A

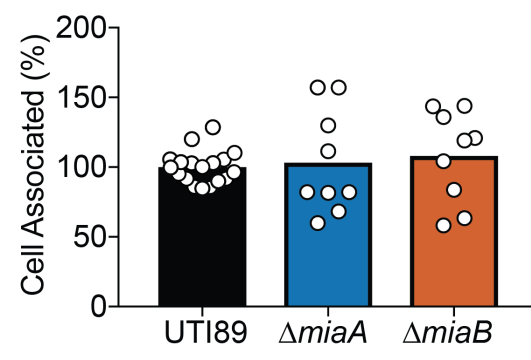

B

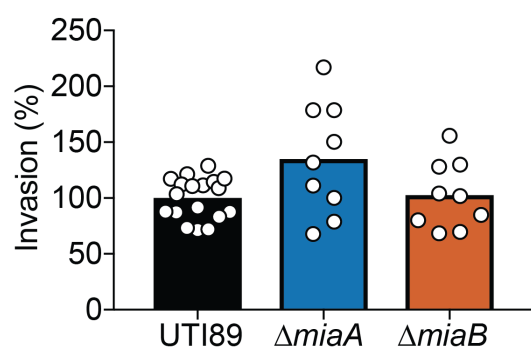

C

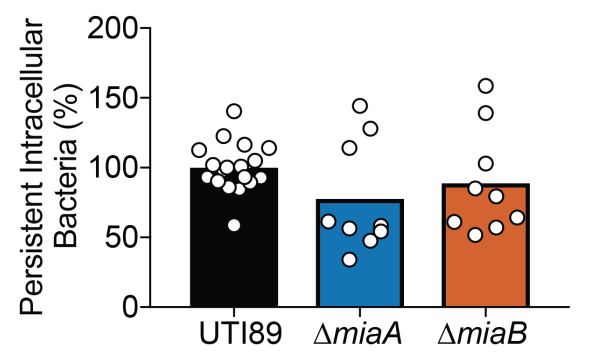


1345 Supplemental Figure S2. MiaA promotes ExPEC colonization and persistence

1346 within the murine bladder.

1347 (A and B) The bladders of adult female CBA/J mice were inoculated via transurethral

1348 injections with $\sim 10^{7}$ CFU of wild-type UTI89 or UTI89AmiaA. Mice were sacrificed (A) 6

1349 hours or (B) 9 days later and bacterial titers within the bladders were determined by

1350 plating tissue homogenates.

1351 (C) Graph shows bacterial titers recovered from the bladders of adult female $\mathrm{C} 3 \mathrm{H} / \mathrm{HeJ}$

1352 mice 3 days after inoculation with UTI89 or UTI89 $\triangle$ miaA.

1353 Bars in all graphs denote median values. ${ }^{*}, P<0.05$ by Mann Whitney $\mathrm{U}$ tests; $n \geq 9$ mice

1354 per group.
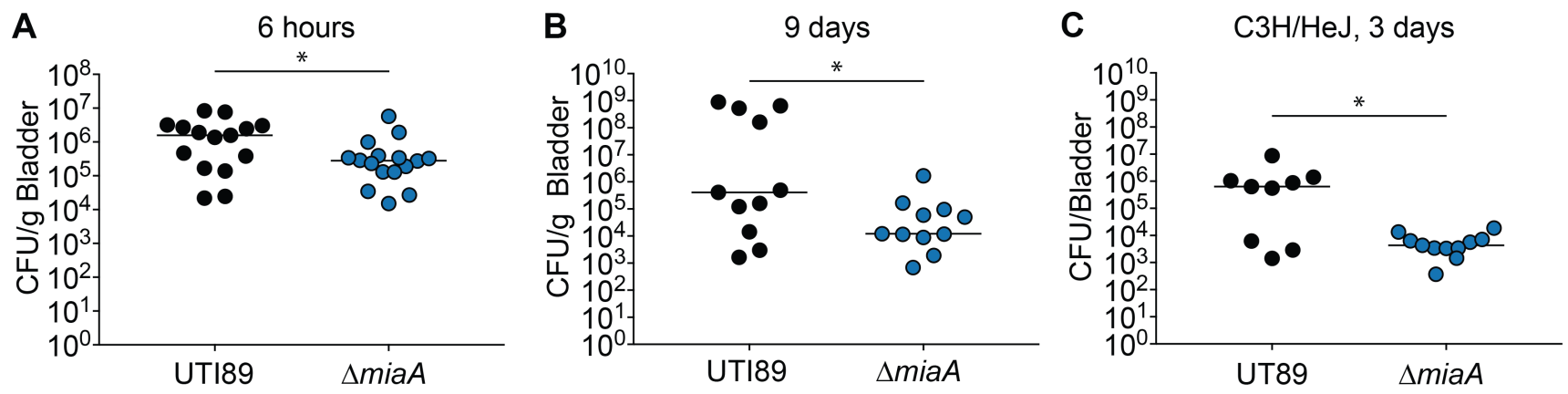


\section{Supplemental Figure S3. MiaA promotes ExPEC fitness in a mouse model of}

1356 sepsis.

1357 Adult female C57BI/6 mice were inoculated via i.p. injections with $10^{7}-10^{8} \mathrm{CFU}$ of UTI89,

$1358 \mathrm{UTI} 89 \Delta m i a A$, or UTI89 $\triangle m i a B$ and 6 hours later bacterial titers were present in the (A)

1359 kidneys, (B) spleen, and (C) liver were determined by plating tissue homogenates. ${ }^{*}, P<$

13600.05 and $^{* *}, P>0.01$ by Mann Whitney $U$ tests; $n=10$ mice per group.
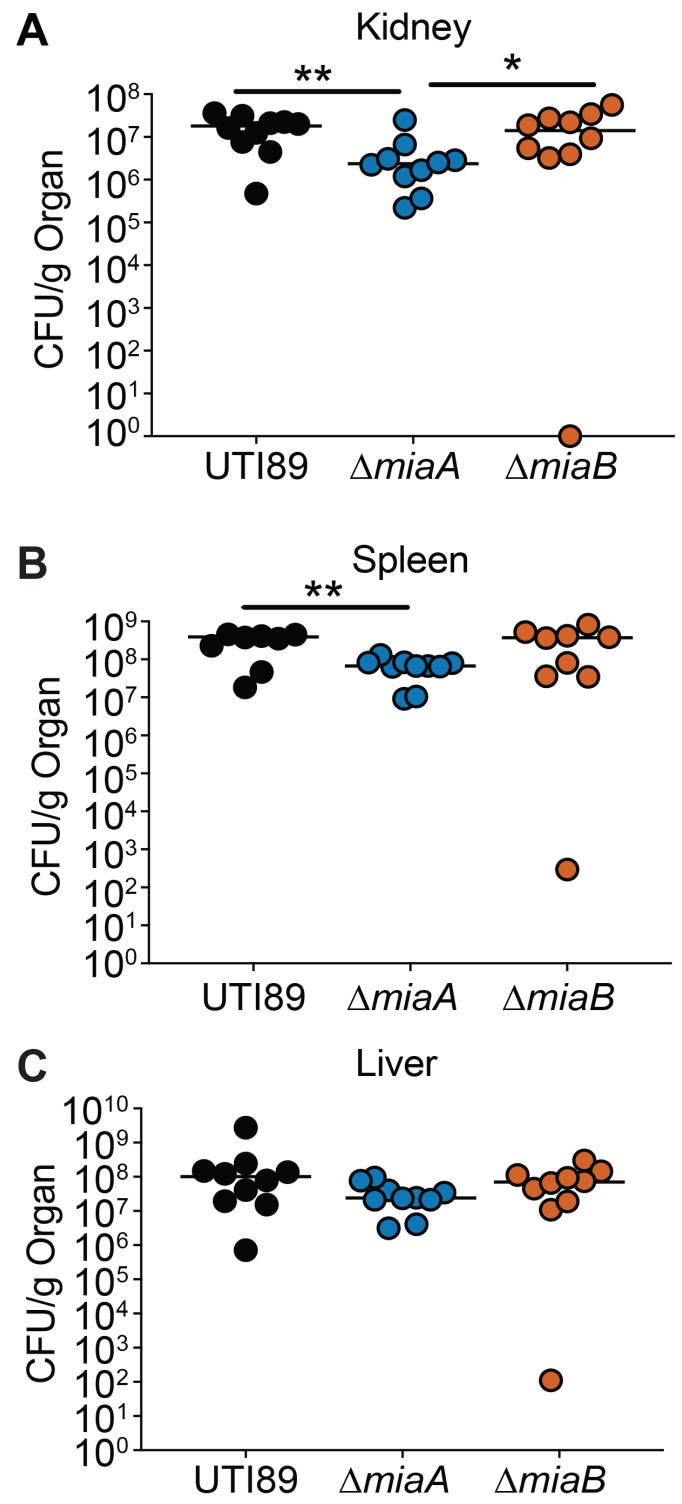
1361 Supplemental Figure S4. The $\mathrm{ms}^{2} \mathrm{i}^{6} \mathrm{~A}$ modification is missing in UTI89 $\Delta \mathrm{miaB}$.

1362 RNA was collected from wild-type UTI89 and UTI89 $\triangle$ miaB after reaching an OD $_{600}$ of 0.5

1363 in shaking LB cultures. Relative levels of $\mathrm{i}^{6} \mathrm{~A}$ and $\mathrm{ms}^{2}{ }^{2}{ }^{6} \mathrm{~A}$ were determined by LC-MS. ${ }^{* * * *}$,

$1364 P<0.0001$ as determined an unpaired $t$ test; $\mathrm{n}=5$ independent replicates.

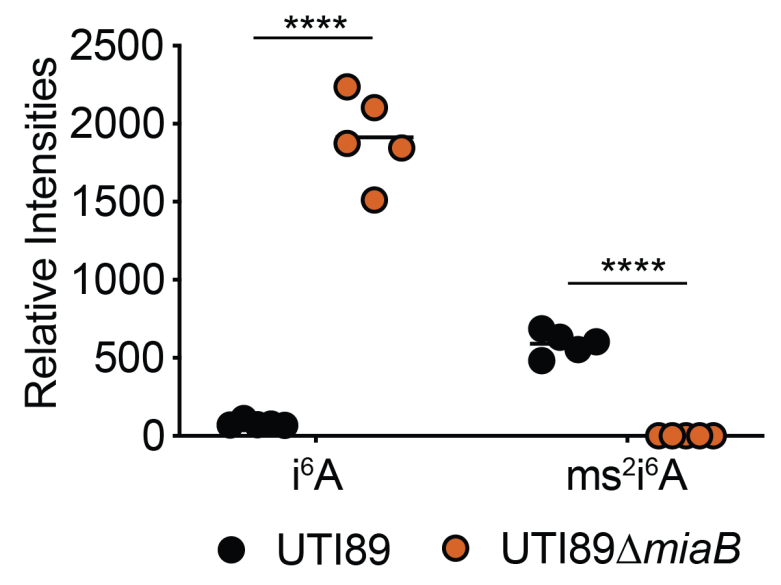


1365 Supplemental Figure S5. Overproduction of MiaB does not affect growth of UTI89

1366 under stressful conditions.

1367 Graphs show growth curves of UTI89 carrying pMiaB Ptac $_{\text {or }}$ or the control plasmid pRR48 in

1368 (A) LB, (B) $1 \mathrm{mM} \mathrm{MV}$, (C) $5 \% \mathrm{NaCl}$, (D) MES-LB, and (E) $1 \mathrm{mM}$ ASN. To overexpress

1369 MiaB, 1 mM IPTG was added to UTI89/pMiaB Ptac. Data are representative of three or

1370 more independent experiments, each done in quadruplicate.

A

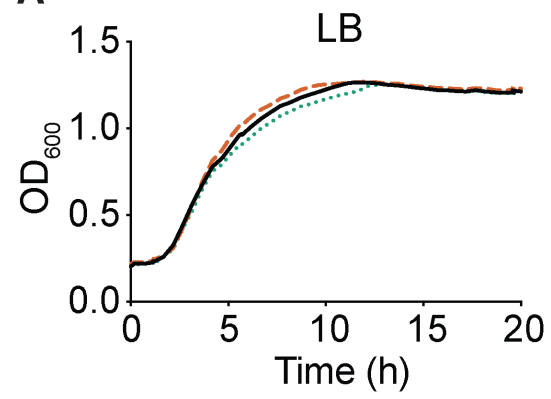

-- UTI89/PMiaB ${ }_{\text {Prac }} 0$ mM IPTG

.... UTI89/pMiaB prac 1 mM IPTG

- UTI89/pRR48
B

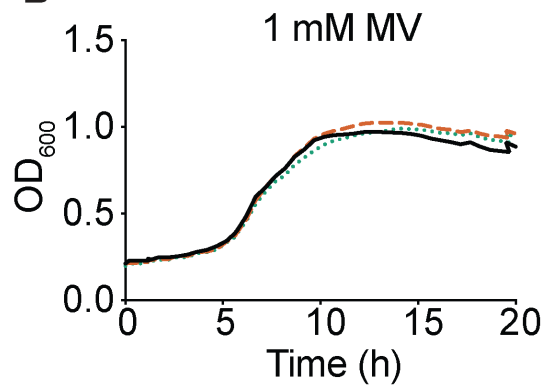

D

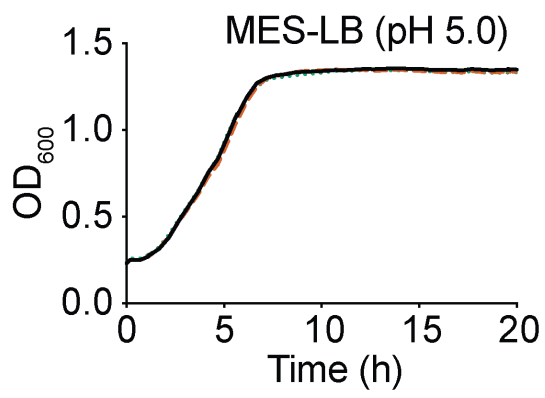

C

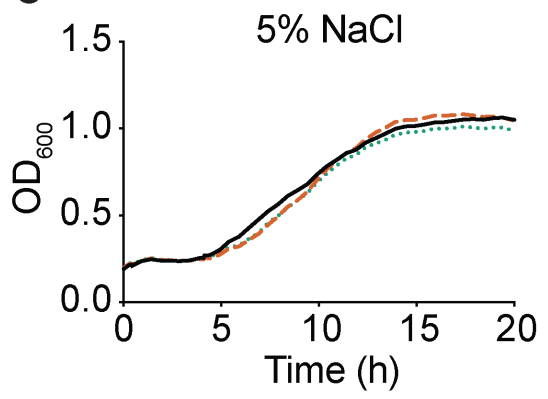

E

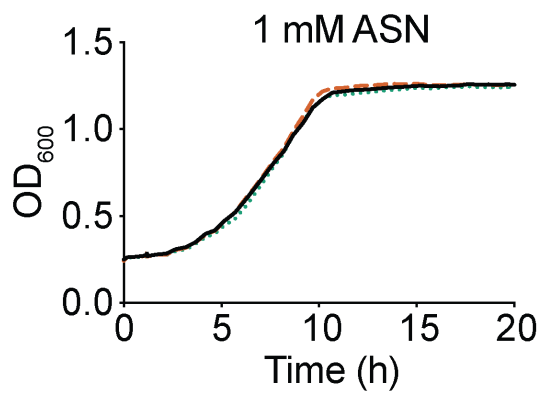


1371 Supplemental Figure S6. MiaA modulates ExPEC motility.

1372 (A - D) Graphs indicate the spread of UTI89 (black lines), UTI89 $\triangle m i a A$ (dotted blue lines),

1373 and UTI89 $\triangle m i a B$ (dashed red lines) on (A and C) LB and (B and D) M9 swim motility

1374 plates incubated at $37^{\circ} \mathrm{C}$. Shown are mean values \pm SD from three independent 1375 experiments done in triplicate. Swim rates $( \pm$ SD) for wild-type UTI89 and UTI89 $\Delta$ miaA 1376 on LB and M9 swim plates are indicated below the graphs in $(A)$ and $(B) .{ }^{*}, P<0.05 ;{ }^{* *}$; $1377 P<0.01 ;{ }^{* * *}, P<0.001 ;{ }^{* * *}, P<0.0001$ versus wild-type UTI89, as determined by

1378 unpaired $t$ tests; $\mathrm{n} \geq 4$ independent replicates. (E) Representative image showing the 1379 spread of UTI89/pRR48, UTI89/pMiaB Ptac, $_{\text {, and UTI89/pMiaA }}$ Ptac 6 hours after inoculation 1380 onto an LB swim plate containing 1 mM IPTG and ampicillin.
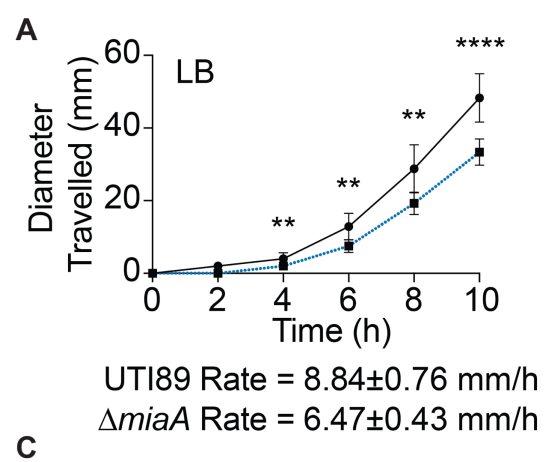

C

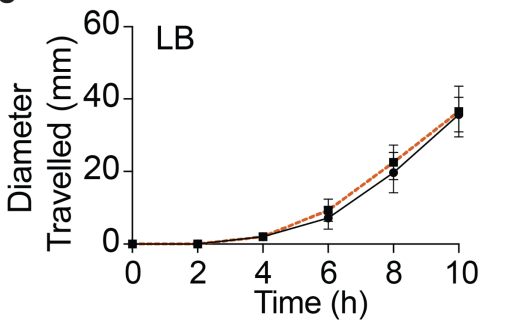

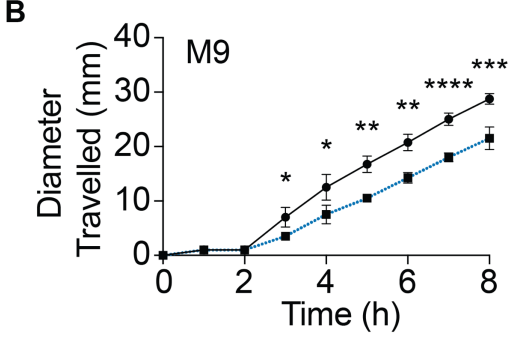

UTI89 Rate $=4.35 \pm 0.17 \mathrm{~mm} / \mathrm{h}$

D $\triangle m i a A$ Rate $=3.6 \pm 0.45 \mathrm{~mm} / \mathrm{h}$

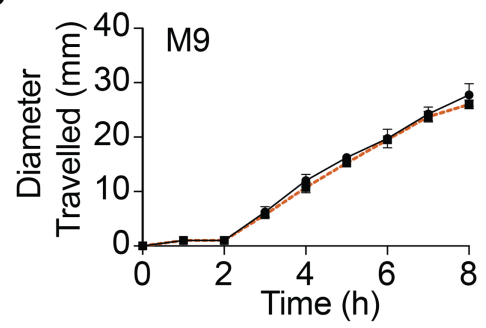

- UTI89 -UTI89 $\triangle m i a A-U T I 89 \Delta m i a B$

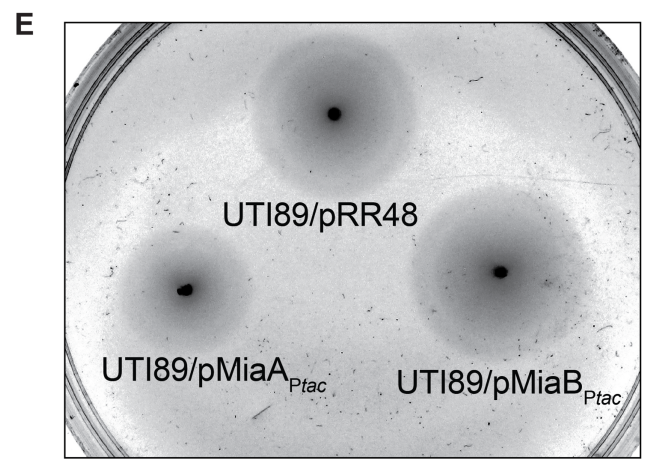




\section{Supplemental Figure S7. UTI89 $\triangle m i a A$ is has increased sensitivity to acid stress.}

1382 After reaching mid-logarithmic growth phase in LB, wild-type UTI89, UTI89 $\triangle m i a A$, and

1383 UTI89 $\triangle m i a B$ were exposed to acidic stress $(\mathrm{pH} 3.0)$ for 30 min. Following washes in PBS,

1384 surviving bacteria were enumerated by dilution plating. Titers are normalized to input.

1385 Biological replicates are connected by lines. ${ }^{*}, P<0.05$ by paired $t$ tests.

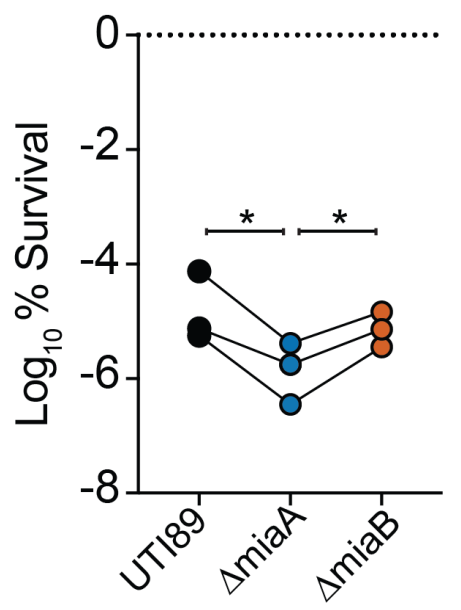


1386 Supplemental Figure S8. Expression of RpoS or Hfq does not rescue growth of 1387 UTI89 $\triangle m i a A$ in the presence of high salt stress.

1388 (A - C) Western blots of RpoS and Flag-tagged Hfq in UTI89 and UTI89 $\Delta$ miaA carrying 1389 pRpoS $\mathrm{Ptac}_{\mathrm{P}}$, pHfqptac, or the empty vector pRR48 following growth to stationary phase in

1390 LB or LB with 1 mM IPTG, as indicated. As a loading control, blots were also probed with

1391 anti-E. coli antibody. A shorter exposure was used for the blot shown in (B), making the

1392 RpoS band from UTI89/pRR48 notably lighter than the one shown in (A). Blots are

1393 representative of three independent experiments.

1394 (D and E) Curves show growth of the UTI89 and UTI89 $\Delta$ miaA with the empty vector

1395 pRR48 or plasmids for IPTG-inducible expression of RpoS or Flag-tagged Hfq in LB $+5 \%$

$1396 \mathrm{NaCl}$. Cultures were grown shaking at $37^{\circ} \mathrm{C}$ with IPTG added in ten-fold increments from

13970 to $1000 \mu \mathrm{M}$, as indicated. Each growth curve shows the means of results from a single

1398 experiment and is representative of at least three independent experiments performed in

1399 quadruplicate.

A

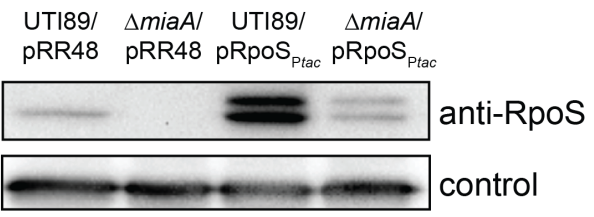

B
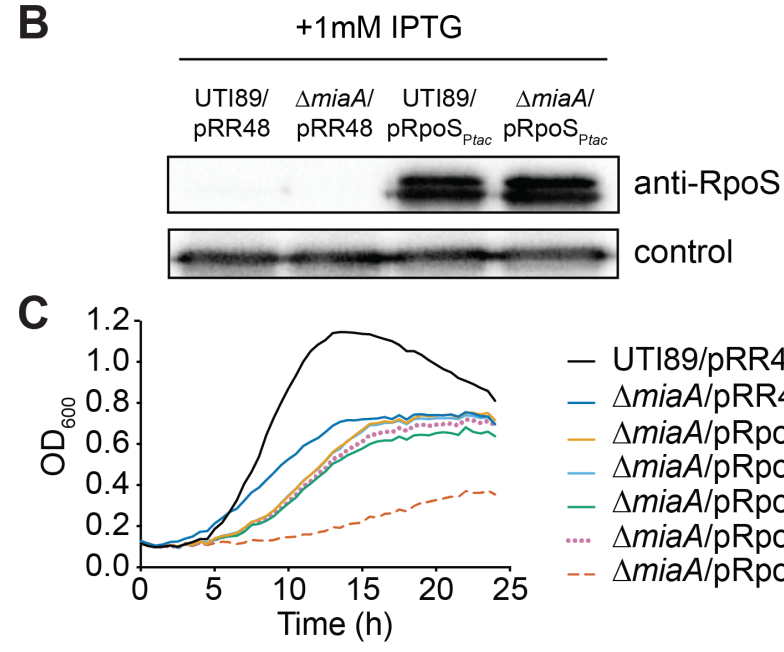

- UTI89/pRR48

- $\triangle m i a A / p R R 48$

- $\Delta m i a A / p R p o S$

- $\triangle m i a A / p R p o S$

- $\Delta m i a A / p R p o S_{\text {Ptac }}^{\text {Ptac }}$

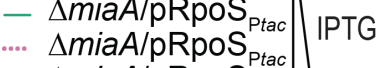

$\begin{array}{ll}\text {... } & \Delta m i a A / p R p o S_{\text {Ptac }} \\ \text {-- } & \Delta m i a A / p R p o S_{\text {tac }}\end{array}$
D

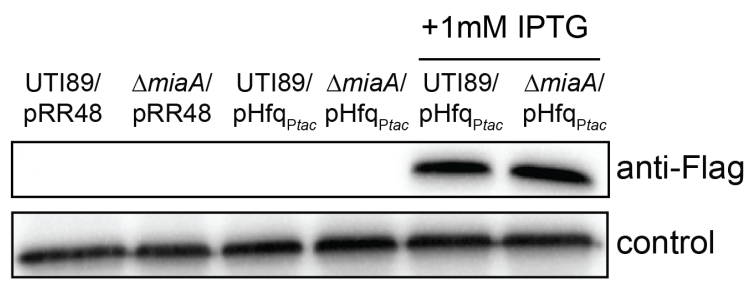

E

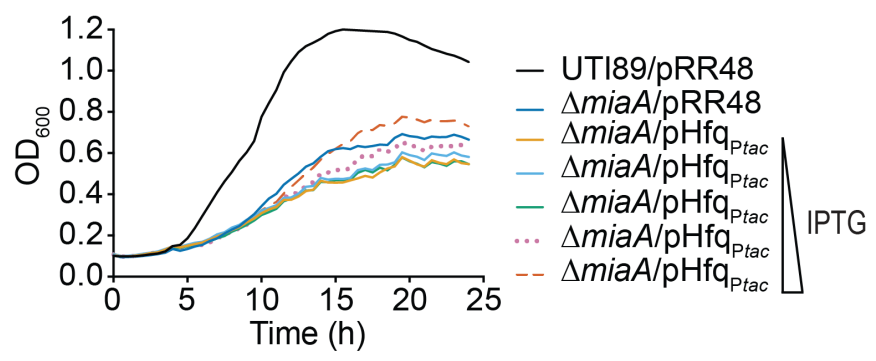


1400 Supplemental Figure S9. Methyl viologen and EDTA alter MiaA levels.

1401 (A) Top panel shows schematic of the experimental setup. UTI89/pMiaA-Flagnat was

1402 diluted from overnight cultures into fresh LB and grown shaking for 2.5 hours at $37^{\circ} \mathrm{C}$ prior

1403 to resuspension in LB, $\mathrm{LB}+5 \% \mathrm{NaCl}$, or LB $+1 \mathrm{mM}$ EDTA. Incubations were continued

1404 for another hour before samples were collected and analyzed by western blots.

1405 (B and C) Blots were probed using anti-Flag (MiaA-Flag) and anti-E. coli (loading control, 1406 bottom) antibodies.

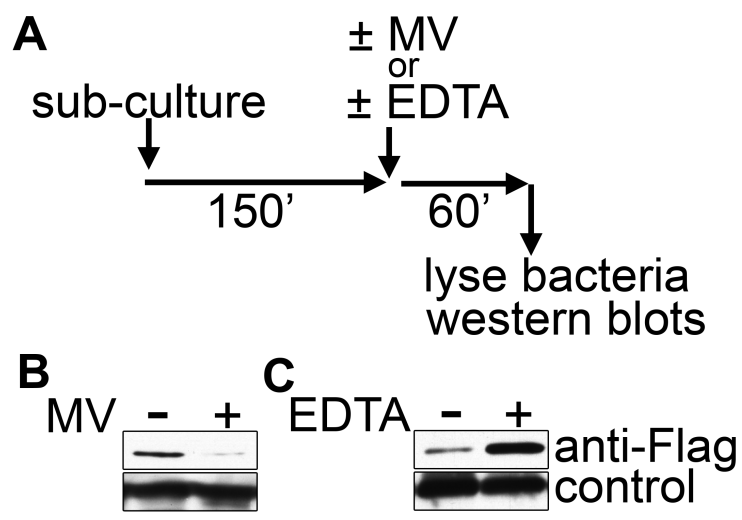


1407 Supplemental Figure S10. UTI89 $\Delta$ miaA is more sensitive to gentamicin than the

1408 wildtype UTI89.

1409 Bars in graph indicate mean MIC values $( \pm$ SD) determined from three independent

1410 Etests. $P$-value was determined by an unpaired Student's $t$ test.

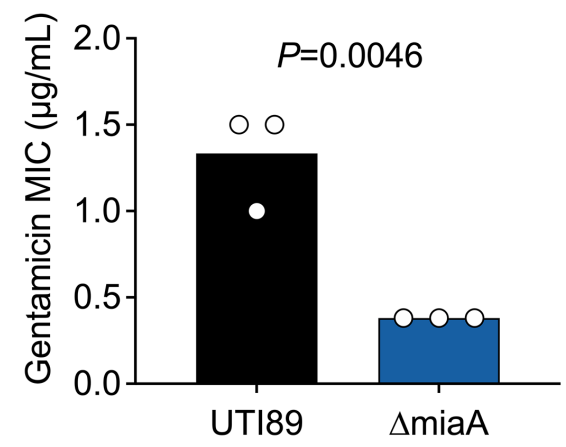


Supplemental Table S1. Bacterial strains and plasmids

\begin{tabular}{|c|c|c|}
\hline $\begin{array}{l}\text { Strain or } \\
\text { Plasmid }\end{array}$ & Description & $\begin{array}{l}\text { Source or } \\
\text { Reference }\end{array}$ \\
\hline \multicolumn{3}{|l|}{ Strains } \\
\hline UTI89 & UPEC reference strain, cystitis isolate & {$[52,108]$} \\
\hline UTI89::Kan ${ }^{R}$ & $\begin{array}{l}\text { UTI89 with a } \mathrm{Kan}^{\mathrm{R}} \text { resistance cassette inserted } \\
\text { at the attTn7 site }\end{array}$ & This study \\
\hline UTI89 $\triangle m i a A$ & UTI89 miaA::Cam ${ }^{\mathrm{R}}$ & This study \\
\hline UTI89 $\Delta m i a B$ & UTI89 miaB::Cam ${ }^{R}$ & This study \\
\hline \multicolumn{3}{|l|}{ Plasmids } \\
\hline pACYC184 & Low-copy number plasmid; Tet $^{R}$, Cam $^{R}$ & $\begin{array}{l}\text { New England } \\
\text { Biolabs }\end{array}$ \\
\hline pBAD18 & $\begin{array}{l}\text { Arabinose-inducible bacterial expression } \\
\text { plasmid; } A m p^{R}\end{array}$ & [109] \\
\hline pBAD33 & $\begin{array}{l}\text { Arabinose-inducible bacterial expression } \\
\text { plasmid; Cam }{ }^{\mathrm{R}}\end{array}$ & [109] \\
\hline pKD3 & $\begin{array}{l}\text { Carries FRT-flanked Cam }{ }^{R} \text { cassette; template } \\
\text { for use in lambda-Red-mediated recombination }\end{array}$ & [48] \\
\hline pKD4 & $\begin{array}{l}\text { Carries FRT-flanked Kan }{ }^{R} \text { cassette; template } \\
\text { for use in lambda-Red-mediated recombination }\end{array}$ & [48] \\
\hline pKM208 & $\begin{array}{l}\text { IPTG-inducible lambda Red recombinase } \\
\text { expression plasmid; } A m p^{R}\end{array}$ & [49] \\
\hline pRR48 & $\begin{array}{l}\text { Contains IPTG-inducible } P_{t a c} \text { promoter } \\
\text { upstream of the MCS; } A m p^{R}\end{array}$ & [110] \\
\hline $\mathrm{pHfq} \mathrm{ptac}_{\mathrm{p}}$ & $\begin{array}{l}\text { hfq from UTI89 cloned with c-terminal } 6 x \mathrm{His} \\
\text { and FLAG tags into Pstl, Hindlll sites of } \\
\text { pRR48; Amp }\end{array}$ & This Study \\
\hline pRpoS $S_{\text {Ptac }}$ & $\begin{array}{l}\text { RpoS cloned from UTI89 into Pstl, Hindlll sites } \\
\text { of pRR48; Amp }\end{array}$ & [62] \\
\hline pMiaAptac & $\begin{array}{l}\text { miaA cloned from UTI89 into Pstl, Kpnl sites of } \\
\text { pRR48; Amp }{ }^{R}\end{array}$ & This study \\
\hline pMiaB Ptac & $\begin{array}{l}\text { miaB cloned from UTI89 into Pstl, Kpnl sites of } \\
\text { pRR48; AmpR }\end{array}$ & This study \\
\hline $\mathrm{pMiaA}_{\text {nat }}$ & $\begin{array}{l}\text { miaA plus } 200 \text { bp of flanking sequences cloned } \\
\text { from UTI89 into the EcoR1 site of pACYC184; } \\
\text { Tet }^{R}\end{array}$ & This study \\
\hline pMiaA-Flag nat & $\begin{array}{l}\text { pACYC184-derived plasmid encoding MiaA } \\
\text { with } N \text {-terminal Flag tag plus linker under } \\
\text { control of native miaA promoter; } \text { Tet }^{R}\end{array}$ & This study \\
\hline
\end{tabular}




\begin{tabular}{|c|c|c|}
\hline p2Luc-HIV & $\begin{array}{l}\text { Eukaryotic reporter construct with the HIV gag- } \\
\text { pol frameshift region inserted between the } \\
\text { renilla and firefly luciferase genes. The HIV } \\
\text { linker sequence contains a } 2 \text {-nucleotide } \\
\text { insertion resulting in a stop codon located } 6 \\
\text { codons after the start of firefly luciferase gene. } \\
\text { The firefly gene is in a - } 1 \text { frame relative to the } \\
\text { upstream renilla gene; Amp }{ }^{R}\end{array}$ & Derived from [76] \\
\hline p2Luc-HIV-IF & $\begin{array}{l}\text { Control for p2Luc-HIV. The HIV gag-pol linker } \\
\text { was altered to keep the renilla and firefly } \\
\text { luciferases in-frame; Amp }{ }^{R} \text {. }\end{array}$ & Derived from [76] \\
\hline p2Lucaz1 & $\begin{array}{l}\text { Eukaryotic reporter construct with the Az1 } \\
\text { frameshift region inserted between the renilla } \\
\text { and firefly luciferase genes. The Az1 linker } \\
\text { sequence contains a stop codon positioned in- } \\
\text { frame so that a }+1 \text { frameshift must occur for } \\
\text { read-through expression of firefly luciferase; } \\
\text { Amp }^{R}\end{array}$ & [75] \\
\hline p2Lucaz1-IF & $\begin{array}{l}\text { Control for } p 2 L u c a z 1 \text {. The } A z \text { linker was } \\
\text { altered to keep the renilla and firefly luciferases } \\
\text { in-frame; } A m p^{R}\end{array}$ & [75] \\
\hline pCWR42-CamR & $\begin{array}{l}\text { Dual luciferase reporter with in-frame Az1 } \\
\text { linker cloned from p2Lucaz1-IF into pBAD33. } \\
\text { Has a Shine-Dalgarno sequence and is under } \\
\text { control of the arabinose-inducible PBAD } \\
\text { promoter. Control for pCWR43-CamR; Cam }\end{array}$ & This study \\
\hline pCWR42-AmpR & $\begin{array}{l}\text { Dual luciferase reporter with in-frame Az1 } \\
\text { linker cloned from p2Lucaz1-IF into pBAD18. } \\
\text { Has a Shine-Dalgarno sequence and is under } \\
\text { control of the arabinose-inducible } P_{B A D} \\
\text { promoter. Control for pCWR43-AmpR; Amp }\end{array}$ & This study \\
\hline pCWR43-CamR & $\begin{array}{l}\text { Dual luciferase reporter with Az1 linker cloned } \\
\text { from p2Lucaz1 into pBAD33. Has a Shine- } \\
\text { Dalgarno sequence and is under control of the } \\
\text { arabinose-inducible PBAD promoter; Cam }{ }^{R}\end{array}$ & This study \\
\hline pCWR43-AmpR & $\begin{array}{l}\text { Dual luciferase reporter with Az1 linker cloned } \\
\text { from p2Lucaz1 into pBAD18. Has a Shine- } \\
\text { Dalgarno sequence and is under control of the } \\
\text { arabinose-inducible PBAD promoter; Amp }{ }^{R}\end{array}$ & This study \\
\hline pCWR44-CamR & $\begin{array}{l}\text { Dual luciferase reporter with in-frame HIV } \\
\text { linker cloned from p2Luc-HIV-IF into pBAD33. } \\
\text { Has a Shine-Dalgarno sequence and is under } \\
\text { control of the arabinose-inducible PBAD } \\
\text { promoter. Control for pCWR45-Cam; Cam }{ }^{R}\end{array}$ & This study \\
\hline
\end{tabular}


pCWR44-AmpR Dual luciferase reporter with in-frame HIV linker cloned from p2Luc-HIV-IF into pBAD18. Has a Shine-Dalgarno sequence and is under control of the arabinose-inducible $P_{B A D}$ promoter. Control for pCWR45-Amp; Amp ${ }^{R}$

pCWR45-CamR Dual luciferase reporter with HIV linker cloned from p2Luc-HIV into pBAD33. Has a ShineDalgarno sequence and is under control of the arabinose-inducible $\mathrm{P}_{\mathrm{BAD}}$ promoter; $\mathrm{Cam}^{\mathrm{R}}$

pCWR45-AmpR Dual luciferase reporter with HIV linker cloned from p2Luc-HIV into pBAD18. Has a ShineDalgarno sequence and is under control of the arabinose-inducible $\mathrm{P}_{\mathrm{BAD}}$ promoter; $\mathrm{Cam}^{\mathrm{R}}$
This study

This study

This study 
Supplemental Table S2. Primers used in this study

\begin{tabular}{|c|c|}
\hline Primer Name ${ }^{a}$ & Sequence $\left(5^{\prime}-3^{\prime}\right)^{b}$ \\
\hline \multicolumn{2}{|l|}{ Cloning primers } \\
\hline MiaA-pRR48-F & CGCGCTGCAGATGAGTGATATCAGTAAGGCG \\
\hline MiaA-pRR48-R & CGGCGGTACCTCAGCCTGCGATAGCACCAAC \\
\hline MiaB-pRR48-F & CGCG $\overline{\overline{C T G C A G}}$ ATGACCAAAA AACTCCATAT TAAAACC \\
\hline MiaB-pRR48-R & CGGCGGTACCGAATTACGGCTGATAATAAC \\
\hline $\begin{array}{l}\text { MiaA-Flag- } \\
\text { pACYC184-F }\end{array}$ & 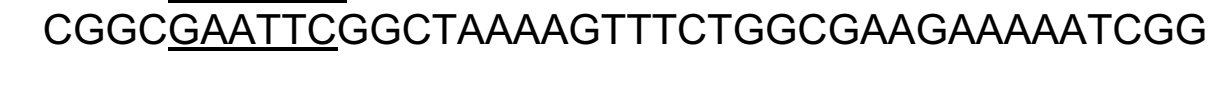 \\
\hline $\begin{array}{l}\text { MiaA-Flag- } \\
\text { DACYC184-R }\end{array}$ & $\begin{array}{l}\text { CGCGGAATTCCTATCCCTTATCGTCGTCATCCTTGTAGTCT } \\
\text { GGTCCTCCTCCTCC GCCTGCGATAGCACCAACAAC }\end{array}$ \\
\hline MiaA-pACYC184-F & CGCGGAATTCGCCCCTTAGCCATTCTCTCTTTTCCTTATATG \\
\hline MiaA-pACYC184-R & 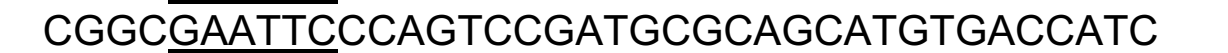 \\
\hline Hfq-pRR48-Pstl & CATACCTGCAGATGGCTAAGGGGCAATCTT \\
\hline $\begin{array}{l}\text { Hfq-CFLAG-his- } \\
\text { Hindll }\end{array}$ & $\begin{array}{l}\text { CATACAAGCTTCTAGTGGTGGTGGTGGTGGTGTCCCTTATC } \\
\text { GTCGTCATCCTTGTAGTCTCC TTCGGTTTCTTCGCTGTCCT }\end{array}$ \\
\hline p2Luc-F & $\begin{array}{l}\text { GCCGGGGTACCAGGAGGTCAGTCAGATGACTTCGAAAGTT } \\
\text { TATGATCCAG }\end{array}$ \\
\hline p2Luc-R & GCCGGAAGCTTTTTACAATTTGGACTTTCCGCCC \\
\hline \multicolumn{2}{|c|}{ Knockout, insertion, and confirmation primers } \\
\hline attTn7KanR-KI-F & $\begin{array}{l}\text { TCTGGCGTAGCCTGGGAGTTATTGCCGGATGCGATGCTGGT } \\
\text { GTGTAGGCTGGAGCTGCTTCG }\end{array}$ \\
\hline attTn7KanR-KI-R & $\begin{array}{l}\text { TCACGTAAAAAAACGTCTAATCCGTAGACCGGATAAGAGGCA } \\
\text { TATGAATATCCTCCTTAG }\end{array}$ \\
\hline MiaA-KO-F & $\begin{array}{l}\text { CGATAAAAGCCCTGAAAGATGAGTGATATCAGTAAGGCTGTG } \\
\text { TAGGCTGGAGCTGCTTCG }\end{array}$ \\
\hline MiaA-KO-R & $\begin{array}{l}\text { CGTCTCCTGACGTTTGCGTCAGTTCCGTTAAAGTTTTACCCAT } \\
\text { ATGAATATCCTCCTTAG }\end{array}$ \\
\hline MiaA-KO-Conf-F & GCCGCCGGGTGGTCTGTTAC \\
\hline MiaA-KO-Conf-R & CAGCCTGCGATAGCACCAAC \\
\hline MiaB-KO-F & $\begin{array}{l}\text { CCTGCATTCCTGGCTACTATTTCGCAAGAGCAAGTCGTGTGT } \\
\text { AGGCTGGAGCTGCTTCG }\end{array}$ \\
\hline MiaB-KO-R & $\begin{array}{l}\text { CGGCGGGCCTGAGAATTACGGCTGATAATAACCCACGCCAT } \\
\text { ATGAATATCCTCCTTAG }\end{array}$ \\
\hline MiaB-KO-Conf-F & GCCGACCATTCCTCCGCCGAC \\
\hline MiaB-KO-Conf-R & CATTGTCTGCTGGCTCCAGG \\
\hline \multicolumn{2}{|l|}{$R T$-qPCR primers } \\
\hline $\begin{array}{l}\text { miaA-F } \\
\text { miaA-R } \\
\text { miaB-F }\end{array}$ & $\begin{array}{l}\text { TACGGACTTGCCTTCCATTC } \\
\text { GCGCAAACACCTCGATAAAC } \\
\text { GTAGAAGGTACATCGCGTAAG }\end{array}$ \\
\hline miaB-R & TCGGGTAGACGTCGGTAAT \\
\hline rpoD-F & TTCGTACGCAAGAACGTCTG \\
\hline rpoD-R & AGGTATCGCTGGTTTCGTTG \\
\hline
\end{tabular}

${ }^{a} \mathrm{~F}$, forward primer; $\mathrm{R}$, reverse primer; $\mathrm{KO}$, knockout primer; $\mathrm{KI}$, knock-in primer;

Conf, confirmation primer. ${ }^{\mathrm{b}}$ Added restriction sites in cloning primers underlined. 